\title{
FINITE DIFFERENCE SOLUTION OF THE TIME DEPENDENT \\ NEUTRON GROUP DIFFUSION EQUATIONS
}

by

John S. Hendricks

Allan F. Henry

August, 1975

DEPARTMENT OF NUCLEAR ENGINEERING
MASSACHUSETTS INSTITUTE OF TECHNOLOGY

Cambridge, Massachusetts 02139

Energy Research and Development Administration Report

Contract AT(1 1-1)-2262

Energy Research and Development Administration 


\section{DISCLAIMER}

This report was prepared as an account of work sponsored by an agency of the United States Government. Neither the United States Government nor any agency Thereof, nor any of their employees, makes any warranty, express or implied, or assumes any legal liability or responsibility for the accuracy, completeness, or usefulness of any information, apparatus, product, or process disclosed, or represents that its use would not infringe privately owned rights. Reference herein to any specific commercial product, process, or service by trade name, trademark, manufacturer, or otherwise does not necessarily constitute or imply its endorsement, recommendation, or favoring by the United States Government or any agency thereof. The views and opinions of authors expressed herein do not necessarily state or reflect those of the United States Government or any agency thereof. 


\section{DISCLAIMER}

Portions of this document may be illegible in electronic image products. Images are produced from the best available original document. 
CoO-2262-8

MITNE-176

\section{MASSACHUSETTS INSTITUTE OF TECHNOLOGY}

DEPARTMENT OF NUCLEAR ENGINEERING

Cambridge, Massachusetts 02139

\section{FINITE DIFFERENCE SOLUTION OF THE TIME DEPENDENT}

NEUTRON GROUP DIFFUSION EQUATIONS

by

John S. Hendricks, Allan F. Henry

August, 1975

Energy Research and Development Administration Report

Contract $\mathrm{AT}(11-1)-2262$

Energy Research and Development Administration

\section{NOTICE}

This report was prepared as an account of work This report Whited States Government. Neither sponsored by the nor the United States Energy Development Administration, nor any of Research and Development Administors, their employees, nor any of thes, makes any subcontractors, or their employees, mames any legal warranty, express or implied, or assumes anpleteness liability or responsibility for the accuracy, comp product or or usefulness of any information, apparatus, product or process disclosed, or represents 
FINITE DIFFERENCE SOLUTION OF THE TIME. DEPENDEN,T

NEUTRON GROUP DIFFUSION EQUATIONS

by

John Stanley Hendricks

Submitted to the Department of Nuclear Engineering on August 7 , 1975, in partial fulfillment of the requirements for the degree of Doctor of Philosophy.

\section{$\underline{\text { ABSTRACT }}$}

In this thesis two unrelated topics of reactor physics are examined: the prompt jump approximation and alternating direction checkerboard methods.

In the prompt jump approximation it is assumed that the prompt and delayed neutrons in a nuclear reactor may be described mathematically as being instantaneously in equilibrium with each other. This approximation has been previously applied to the point kinetics reactor model; in this thesis it is applied to the spatially dependent neutron diffusion theory reactor kinetics model.

Alternating direction checkerboard methods are a family of finite difference alternating direction methods which may be used to solve the multigroup, multidimension, time-dependent neutron diffusion equations. The reactor mesh grid is not swept line by line or point by point as in implicit or explicit alternating direction methods; instead, the reactor mesh grid may be thought of as a checkerboard in which all the "red squares" and "black squares" are treated successively. Two members of this family of methods, the ADC and NSADC methods, are at least as good as other alternating direction methods. 
It has been found that the accuracy of implicit and explicit alternating direction methods can be greatly improved by the application of an exponential transformation. This transformation is incompatible with checkerboard methods. Therefore, a new formulation of the exponential transformation has been developed which is compatible with checkerboard methods and at least as good as the former transformation for other alternating direction methods.

Thesis Supervisor: Allan F. Henry

Title: Professor of Nuclear Engineering 
TABLE OF CONTENTS

ABSTRACT 2

LIST OF FIGURES

LIST OF TABLES 9

$\begin{array}{ll}\text { ACKNOWLEDGMENTS } & 10\end{array}$

BIOGRAPHICAL NOTE 11

Chapter 1. INTRODUCTION 13

1.1 Mathematical Models for Reactor Kinetics 13

1.2. The Multigroup Neutron Diffusion 14 Equation

1.3 Review of Solution. Techniques 17

1. 4 Theta Methods and AIternating Direction 20 Methods

1. 4 a Theta Methods 23

1.4b Alternating Direction Methods 25

1.5 Proposed Methods 27

Chapter 2. THE PROMPT JUMP APPROXIMATION IN 29

2.1 A Point Kinetics Explanation of the Prompt 31 Jump Approximation

2.Ia Review of the Point Kinetics 31

2.Ib Review of the Solution of the. 33

Point Kinetics Equations

2.Ic The Prompt Jump Approximation Applied to the Point Kinetics Model 
2.1d The Solution of the Prompt 38 Jump Point Kinetics

Equations

2.1e The Effect of the Prompt Jump 43

Approximation on Time Step

Size -- A Point Kinetics

Argument

2.If The Two-Level Kinetics Matrix 48

Discretization

2.2 The Spatially Dependent Prompt Jump 55

Approximation

2.2a A One-Group, One-Dimensional . 56

Theta Method Neutron Diffusion

Equation

2.2b Application of the Prompt Jump 59

Approximation to the Spatially

Dependent Finite Difference

Equations

2.3 Numerical Results . 61

2.4 Conclusions 69

Chapter 3. THE ALTERNATING DIRECTION CHECKERBOARD 71 METHOD

3.1 Review of Alternating Direction Methods 71

3.1a Implicit Methods 73

3.Ib Explicit Methods 79

3.1c The Exponential Transformation 82

3.1d Truncation Errors 86

3.le Comparison of Some Symmetric and 89

Nonsymmetric Methods

3.2 The Alternating Direction Checkerboard 93

Method and a New Formulation of the

Frequency Transform 
3.2a The Checkerboard Matrix Splitting 93

3. 2b The Two-Level Frequency Transform 98

3.2c Two-Level Frequenicy Transform and 102

3.2d Computational Considerations of

3.2e Alternative Checkerboard Methods 110

3.3 Numerical Properties 114

3.3a Steady State Behavior 114

3. 3b Consistency 116

3.3c Stability 121

3.4 Numerical Results 134

3.4a Numerical Assessment of the ADC 135

Method and Several Variations of the ADC Method

3.4b Comparison of Frequency Transforms 145

3.4c Comparison of Alternative Methods 148

3.4d A Final Example 149

3.5 Conclusions 153

REFERENCES 156

Appendix A. RELEVANT EQUATIONS 159

A.1 The Neutron Diffusion Equation 159

A.2 The Point Kinetics Equations 160

A.3 The Multigroup Neutron Diffusion 165

Equations

A.3a The One-Group, One-Dimension 168

Neutron Diffusion Equation

A. 3b An Alternative Form of the

Multigroup Neutron Diffusion Equations 
A. 4 The A-Matrix and Flux Vector 171

Appendix B. VECTOR AND MATRIX NORMS 177

Appendix C. SUPPLEMENTARY STABILITY ANALYSIS 182

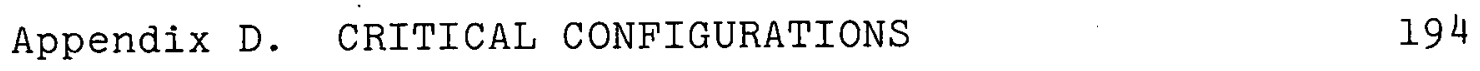




\section{LIST OF FIGURES}

2.1 Neutron Flux Response to a Negative Step 30

2.2 Characteristic Values of the Prompt Jump 41 and Point Kinetics Equations

2.3 Semi-Log Plot of Table 2.1 Data 62

3.1 Reactor Mesh Grid 76

3.2 Checkerboard Reactor Mesh Grid 94 


\section{LIST OF TABLES}

2.1 Test Problem 2.1 62

2.2 Test Problem 2.2 64

2.3 Test Problem 2.3 65

2.4 Test Problem 2.4. 67

3.I Relative Computing Times 107

3.2 ADC Computational Characteristics 111

3.3 Test Problem 3.1 139

3.4 Test Problem $3.2 \quad 141$

3.5 Test Problem 3.3 146

3.6 Test Problem $3.2 \quad 150$ 


\section{ACKNOWLEDGMENTS}

The author wishes to express his appreciation to Professor Allan F. Henry for his support, guidance, and enthusiasm provided during the course of this work. The author also wishes to thank Professor Kent F. Hansen for his comments and suggestions.

Fellowship support for the author was provided by a National Science Foundation Graduate Fellowship Award. Computational work was funded by the U.S. Energy Research and Development Administration. 


\section{BIOGRAPHICAL NOTE}

John S. Hendricks

While attending secondary school he delivered newspapers and played trombone in school bands. He was graduated from Hollywood High School in 1967 and then enrolled at Los Angeles Valley College. While at Valley College, Mr. Hendricks was president of the campus service club and of the campus honor society. He was chosen "Man of the Year, 1968-69" and was graduated Magna Cum Laude. During this time he also worked as a printer, lithographer, and playground director.

In $1969 \mathrm{Mr}$. Hendricks enrolled at the University of California at Los Angeles and continued working as a playground director. In 1970 he was honored as the "Most Outstanding Playground Director, Valley East District, Los Angeles City Schools". At UCLA he was Vice-President of Alpha Phi Omega, National Service Fraternity, President of Tau Beta Pi, National Engineering Honor Society, Secretary of the American Nuclear Society Student Branch, and a representative to ESUC, the engineering student council. In 1972 he was graduated Summa Cum Laude with a B.S. Degree in Engineering and was honored as "The Most Outstanding Bachelors Degree Candidate" of the School of 
Engineering. At the same time he received his M.S. Degree in Nuclear Engineering and represented his class of 5,000 graduate students as the University Commencement speaker at the 1972 UCLA commencement exercises. During the summers of 1971, 1972 and 1973, Mr. Hendricks worked at Los Alamos Scientific Laboratory in New Mexico. His work there included nuclear safeguards, nondestructive assay and nuclear cross section evaluation and processing. At Los Alamos he also served as a church organist and belonged to Toastmasters International.

In $1972 \mathrm{Mr}$. Hendricks

received a National Science Foundation Graduate Fellowship Award, and enrolled at the Massachusetts Institute of Technology. At MIT he served on the Graduate Student Council, the Committee on Commencement, the Corporation Joint Advisory Committee and the Compton AwardsCommittee. He was a trustee of the MIT Community Service Fund, Vice-President of the American Nuclear Society Student Branch, and President of the Graduate Student Council. He was also a lector and hymn leader at his church in Somerville, Massachusetts. 


\section{Chapter 1}

\section{INTRODUCTION}

\subsection{Mathematical Models for Reactor Kinetics Problems}

Nuclear reactor kinetics is the study of the time dependent behavior of neutrons in a reactor. Knowledge of how the neutron population in a reactor will respond to various perturbations is essential to safe and economical design. Unfortunately, this knowledge is very difficult to obtain.

The neutron population in a reactor may be modeled mathematically in several ways. An exact treatment is the Boltzman equation for neutron transport. Solving this equation is both time consuming and difficult. An analytic solution is usually impossible, and approximate numerical solutions are often too expensive.

A simpler mathematical description of the neutron behavior in a reactor is that of point kinetics for which angular and spatial neutronic properties are assumed constant in time and are integrated out. For many problems angular effects may be neglected but spatial effects may not; any reactor subjected to a nonuniformly applied perturbation will exhibit a nonuniform spatial behavior. Hence point kinetics is usually inadequate for describing the detailed behavior of neutrons in a reactor.

The neutron population of a reactor may also be described by diffusion theory. Complicated angular effects 
are neglected so that the diffusion equation is simpler than the transport equation. Space, time, and energy are treated with sufficient accuracy so that approximate solutions of the diffusion equation are usually satisfactory for most kinetics problems. For this thesis it is assumed that solution of the time, space, and energy dependent neutron diffusion equation is adequate for the reactor kinetics problems of interest.

1.2 The Multigroup Neutron Diffusion Equation The neutron diffusion equation is

$\frac{1}{V(E)} \frac{\partial}{\partial t} \phi(\bar{r}, E, t)=\bar{\nabla} \cdot D(\bar{r}, E, t) \nabla \phi(\bar{r}, E, t)-\Sigma_{t}(\bar{r}, E, t) \phi(\bar{r}, E, t)$.

$+\int_{0}^{\infty}\left[(1-\beta) X(E) \nu \Sigma_{f}\left(\bar{r}, E^{\prime}, t\right)+\Sigma_{s}\left(\bar{r}, E^{\prime} \rightarrow E, t\right)\right] \phi\left(F, E^{\prime}, t\right) d E^{\prime}$.

$+\sum_{i=1}^{I} f_{i}(E) \lambda_{i} C_{i}(\bar{r}, t)+q(\bar{r}, E, t)$

where the delayed neutron precursor concentrations, $c_{i}(\bar{r}, t)$, are found by

$$
\begin{array}{r}
\frac{\partial}{\partial t} C_{i}(\bar{r}, t)=\int_{0}^{\infty} \beta_{i} \nu \Sigma_{f}\left(\bar{r}, E^{\prime}, t\right) \phi\left(\bar{r}, E^{\prime}, t\right) d E^{\prime}-\lambda_{i} C_{i}(\bar{r}, t) \\
i=1,2, \cdots, I
\end{array}
$$

Notation is defined in Appendix A.1.

In order to solve this equation numerically the variables must be discretized in space, time, and energy. 
Different methods discretize time and space in different ways, but most methods treat energy dependence by recasting Eqs. (1.1) in multigroup form. The multigroup neutron diffusion equation, as derived from Eqs. (1.1) in Appendix A.3, is

$$
\begin{gathered}
\frac{1}{v_{g}} \frac{\partial}{\partial t} \phi_{g}(\bar{r}, t)=\bar{\nabla} \cdot D_{g}(\bar{r}, t) \nabla \phi_{g}(\bar{r}, t)+\sum_{g^{\prime}=1}^{G} \sum_{g g^{\prime}}(\bar{r}, t) \phi_{g^{\prime}}(\bar{r}, t) \\
+\sum_{i=1}^{I} f_{g i} C_{i}(\bar{r}, t) \quad g=1,2, \cdots ; G \\
\begin{array}{c}
\frac{\partial}{\partial t} C_{i}(\bar{r}, t)=\sum_{g=1}^{G} P_{i g}(\bar{r}, t) \phi_{g}(\bar{r}, t)-\lambda_{i} C_{i}(\bar{r}, t) \\
i=1,2, \cdots, I
\end{array}
\end{gathered}
$$

Notation is defined in Appendix A.3. These equations are as exact as Eq. (I.I) only if the multigroups constants, $D_{g}(\bar{r}, t), \Sigma_{g g},(\bar{r}, t)$, etc., are known exactly.

The multigroup neutron diffusion equation may be compacted into matrix form as

$$
\frac{\partial}{\partial t} \bar{\Phi}=\underline{a} \bar{\Phi}
$$

where 


$$
\bar{\Phi} \equiv\left[\begin{array}{c}
\phi_{1}(\tilde{r}, t) \\
\phi_{2}(\bar{r}, t) \\
\vdots \\
\phi_{G}(\bar{r}, t) \\
C_{1}(\bar{r}, t) \\
\vdots \\
C_{I}(\bar{r}, t)
\end{array}\right]
$$

and

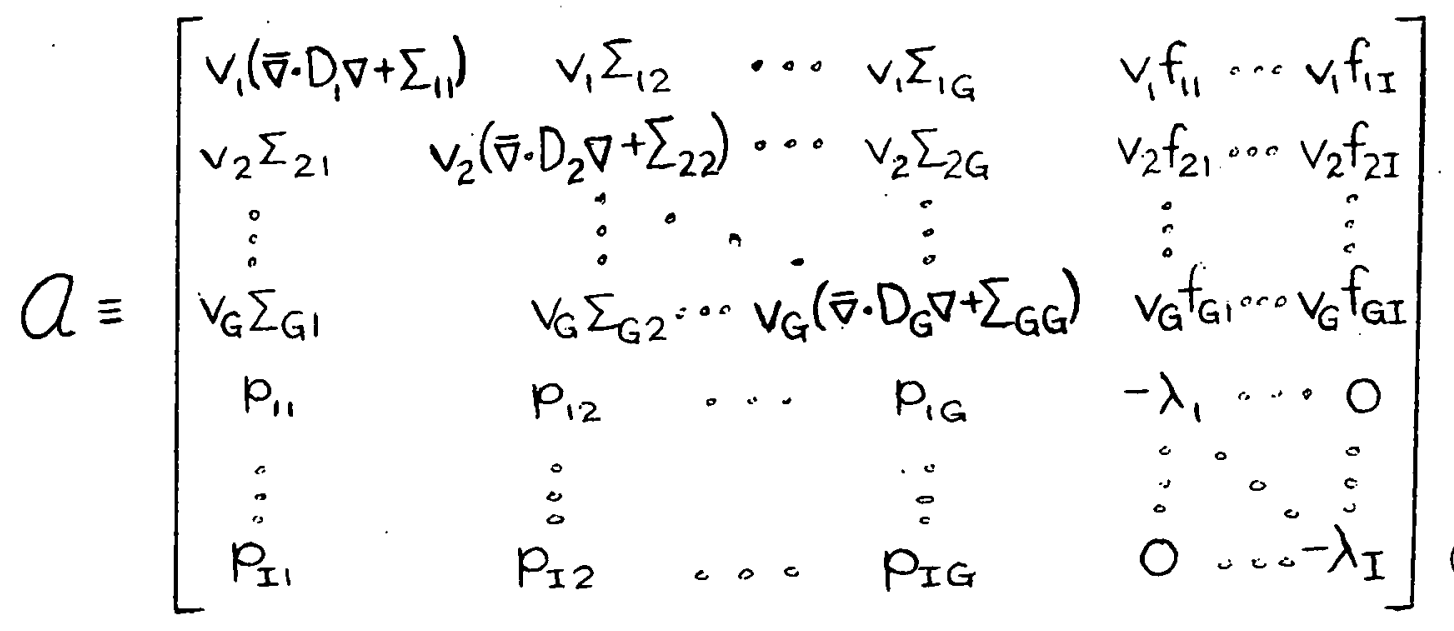

The dependence of $\mathrm{D}_{g}, \Sigma_{g g}{ }^{\prime}, f_{g i}$ and $p_{i g}$ on $(\bar{r}, t)$ is implied. Note that $\underline{a}$ is a matrix of operators.

other forms for $\Phi$ and $\underline{\underline{a}}$ are possible depending upon the ordering of unknowns, the method of solution, and the discretization of space and time. A finite difference form of $\bar{\Phi}$ and $\underline{\underline{a}}$ in which $\underline{\underline{Q}}$ is no longer an operator but rather a matrix of numbers is presented in section 1.4 . Eq. (1.3) is a convenient way of expressing the multigroup neutron diffusion equation without specifying the method of solution. 
Since a general solution of Eq. (1.3) in terms of continuous parameters $\bar{r}$ and $t$ is impractical for most reactor problems of interest, numerical solution techniques are. usually employed. A nuclear reactor may be numerically modeled as a grid of mesh points. Associated with each mesh point are the values of the dependent variables of the neutron diffusion equation: G group fluxes and I precursor concentrations. For a total of $\mathrm{N}$ mesh points there are then $(G+I){ }^{*} N$ unknowns; this is a large number for most applications. Furthermore, the time dependence is discretized and the unknowns must be solved at each step in time. If the time step size, $h$, between each level in time is small, as it usually is, then to model a given transient requires many time steps. Solving many unknowns at each of many time steps requires a large amount of computing effort and becomes quite expensive. As a result much effort has been devoted in recent years to the development of methods that will numerically solve the multigroup neutron diffusion equation as efficiently as possible. Some of these methods are reviewed in the next section.

1.3 Review of Solution Techniques

A solution technique should be as economical as possible while still providing a solution that is sufficiently accurate for the particular application. One way to achieve economy is to reduce the number of unknowns that must be found. Another way is to minimize the number of 
times that the unknowns must be determined; this is accomplished by methods in which large. time step sizes are possible. The methods described in this section either permit large time step sizes and/or a reduction in the number of unknowns.

In finite element methods ${ }^{1,2}$ the spatial flux shape is approximated by piecewise polynomials over spatial mesh regions, or "elements". Finite element methods tena to reduce the number of spatial points at which the flux must be calculated for results of a certain degree of accuracy. The time dependence of the flux may also be approximated by polynomials to increase the time step size. These methods are amenable to mathematical analysis which guarantees convergence; error bounds are readily determined. In nodal methods 3 a reactor is divided into large subregions. A single "node" is then associated with each of these large subregions and assigned gross values that are characteristic of the nuclear properties of the subregion. The number of spatial points at which a solution is sought is thus greatly reduced. These nodes are then coupled together by coupling coefficients. The nodes may be treated as "black boxes" once the coupling coefficients between them are specified. Included among nodal methods are response matrix techniques which are presently under development 4 . 
In modal methods 5 the number of spatial unknowns is reduced by expanding the fluxes as sums of spatial modes with time varying coefficients:

$$
\phi_{g}(\bar{r}, t)=\sum_{k=1}^{K} T_{g k}(t) \phi_{g k}(\bar{r})
$$

where

$$
\begin{aligned}
& \phi_{g}(\bar{r}, t)=\text { multigroup flux } \\
& T_{g k}(t)=\text { coefficient of kth mode } \\
& \phi_{g k}(\bar{r})=\text { kth. spatial mode or "trial function". }
\end{aligned}
$$

Expanding fluxes as sums of spatial modes increases the number of equations to be solved and the number of unknowns at each mesh point. However, the spatial modes describe the flux behavior over large portions of the reactor; therefore, fewer spatial mesh points are required and the total number of unknowns is substantially decreased. The choice of spatial modes is important. In synthesis methods 6,7 spatial modes are chosen to be the fundamental mode of a set of operators, each of which describes the reactor in a different state anticipated at different times during a given transient. Alternatively, the spatial modes may represent predicted flux shapes over subregions of the reactor.

Quasi-static methods ${ }^{8}$ may be thought of as synthesis methods with only one spatial mode. This single mode is recalculated every few time steps by a static flux calculation. Though the number of mesh points required may be 
large the number of times at which the static fluxes must be calculated is small.

Modal methods are characterized by a lack of definitive error bounds. If the number, choice, and frequency of calculation of spatial modes is adequate, then modal methods can be fast and accurate; otherwise they can be erroneous.

In finite difference methods the neutron diffusion equation, Eq. (1.3), is solved directly: fluxes are not approximated by piecewise polynomials, coupled black boxes, or spatial modes as in finite element, nodal, or modal methods. The only assumption made is that differential operators in the diffusion equation may be adquately represented by finite differences. A consequence of this lack of major approximations is that finite difference methods. are characterized by a large number of unknowns. In favor of finite difference methods is the applicability of mathematical analysis which makes fairly definite error estimates possible. Also there are finite difference methods for which large time step sizes are possible; two of these will be described in the next section.

\subsection{Theta Methods and Alternating Direction Methods}

Theta methods and alternating direction methods are two classes of finite difference methods of particular interest to this thesis. In order to describe these methods it is first necessary to examine the spatial and temporal discretization of finite difference methods. 
The spatial discretization of the neutron diffusion equation, Eq. (I.3),

$$
\frac{\partial \bar{\Phi}}{\partial t}=\underline{Q} \bar{\Phi}
$$

is presented in detail in Appendix A.4 and is here briefly summarized. Let a reactor be modeled by a grid of $\mathrm{N}$ spatial mesh points. Then spatial discretization of the $(G+I)$ element vector $\bar{\Phi}$ leads to a $(G+I) x N$ element vector $\bar{\psi}$ and the $(G+I) \times(G+I)$ operator matrix $\underline{a}$ becomes a $(G+I) \times N \times(G+I) \times N$ matrix $\underline{A}$. The diffusion operators, $\bar{\nabla} \dot{D}_{g} \nabla$, are approximated by central differences ${ }^{9}$ so that $\underline{A}$ is a matrix of numbers rather than operators. The spatially discretized multigroup neutron diffusion equation is then

$$
\frac{\partial \bar{\psi}}{\partial t}=\underline{A} \bar{\psi}
$$

As shown in Appendix A.4 for two-dimensions, the matrix A is of the form

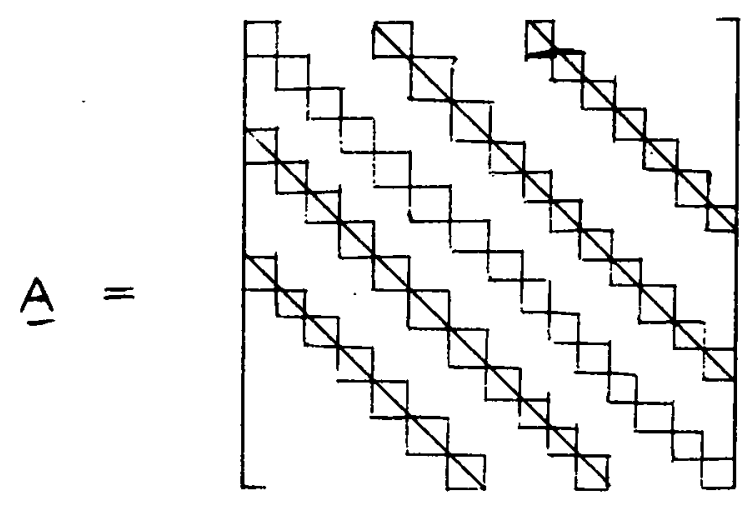


This is a sparse penta-block diagonal matrix. The $\mathrm{N}$ diagonal blocks are dense $(G+I) \times(G+I)$ matrices. The off-diagonal blocks are $(G+I) \times(G+I)$ strictly diagonal matrices, or "stripes". There are two stripes for each spatial dimension. Because of the central difference approximation to the diffusion operators, the number matrix $\underline{A}$ is only a second order approximation 9 to the operator matrix $\underline{a}$.

The temporal discretization for finite difference methods may be carried out as

$$
\frac{\partial \bar{\psi}}{\partial t}=\frac{\bar{\psi}^{n+1}-\bar{\psi}^{n}}{h}
$$

where $\quad \bar{\psi}^{n+1}=$ flux vector at time $t^{n+1}$

$$
\begin{aligned}
\bar{\psi}^{n} & =\text { flux vector at time } t^{n} \\
n & =t^{n+I}-t^{n}=\text { time step size. }
\end{aligned}
$$

For this relation to be substituted into Eq. (1.4a) the flux vector on the right hand side of

$$
\frac{\partial \bar{\psi}}{\partial t}=\underline{A} \bar{\psi}
$$

may also be discretized so that it is evaluated at discrete points in time. To accomplish this, the A-matrix is divided as

$$
\underline{A}=\underline{A}_{1}+\underline{A}_{2}
$$


so that Eq. (1.3a) in finite difference form is

$$
\frac{\bar{\psi}^{n+1}-\bar{\psi}^{n}}{h}=\underline{A}_{1} \bar{\psi}^{n}+\underline{A}_{2} \bar{\psi}^{n+1}
$$

Finite difference methods for which $\underline{A}$ is divided as in

Eq. (1.5) are called splitting techniques. Two successful

classes of splitting techniques are theta methods and

alternating direction methods.

1.4a Theta Methods

For theta methods the choice of $\underline{A}_{1}$ and $\underline{A}_{2}$ is such

that

$$
\underline{A}_{2}=\underline{\theta} \underline{A}
$$

$$
\underline{A}_{1}=(\underline{I}-\underline{\theta}) \underline{A}
$$

or

$$
\frac{\bar{\psi}^{n+1}-\bar{\psi}^{n}}{h}=\underline{\theta} \underline{A} \bar{\psi}^{n+1}+(\underline{I}-\underline{\theta}) \underline{A} \bar{\psi}^{n}
$$

where $\underline{\Theta}$ is a diagonal matrix of elements, $\theta_{i i}$, such that

$$
0 \leqslant \theta_{i i} \leqslant 1
$$


A well known one-dimensional theta method is the WIGLE ${ }^{10}$ method in which the elements $\theta_{i i}$ are calculated at each time step in an attempt to improve accuracy and make large time step sizes possible. By rearranging Eq. (1.7c) as

$$
\bar{\psi}^{n+1}=[I-h \underline{\underline{A}}]^{-1}[I+h(I-\underline{\theta}) \underline{A}] \bar{\psi}^{n}
$$

it is seen that the matrix ( $I$ - h보 $)$ must be inverted at each time step. Except for one-dimensional, one-energy group problems, for which $\underline{A}$ is tridiagonal, the inversion of ( I - h $\underline{\theta A}$ ) must be carried out iteratively. This is equivalent to solving a fixed-source subcritical reactor calculation at each time step and is very time consuming and expensive.

A well known two dimensional theta method is that of TWIGL ${ }^{11}$. In the TWIGL code all elements $\theta_{i i}$ are identical so that $\theta$ is equivalent to a number, $\theta$, hereafter called an implicity constant.

If $\theta=0$ the theta methods become

$$
\frac{\bar{\psi}^{n+1}-\bar{\psi}^{n}}{h}=A \bar{\psi}^{n}
$$

This is known as a fully explicit method. Upon rearranging, 
Eq. (1.8) becomes

$$
\bar{\psi}^{n+1}=(\underline{I}+h \underline{A}) \bar{\psi}^{n}
$$

Because no matrix inversion is required the fully explicit method is very fast. However, it is also numerically unstable unless $h$ is undesirably small 9 .

If $\theta=1$ the theta method becomes

$$
\frac{\bar{\psi}^{n+1}-\bar{\psi}^{n}}{h}=A \bar{\psi}^{n+1}
$$

This is known as a fully implicit method.

For any value of $\theta$ between 0 and 1 the theta method is known as a semi-implicit method. Semi-implicit methods are numerically stable for $\theta \geqslant 1 / 2$. From Eq. (1.7d) it is seen that an iterative matrix inversion is generally required if $\theta>0$. Setting $\theta=1 / 2$ yields the Crank-Nicholson approximation. which has a favorable high order accuracy. Proper selection of $\theta$ makes it possible for semi-implicit methods to solve the neutron diffusion equation efficiently by using large time step sizes.

$1.4 \mathrm{~b}$ Alternating Direction Methods

Alternating direction methods use a two step finite difference approach:

$$
\frac{\bar{\psi}^{n+1}-\bar{\psi}^{n}}{h}=\underline{A}_{1} \bar{\psi}^{n}+\underline{A}_{2} \bar{\psi}^{n+1}
$$




$$
\frac{\bar{\psi}^{n+2}-\bar{\psi}^{n+1}}{h}=\underline{A}_{3} \bar{\psi}^{n+1}+\underline{A}_{4} \bar{\psi}^{n+2}
$$

where

$$
\begin{aligned}
& \underline{A}_{1}+\underline{A}_{2}=\underline{A} \text { and } \underline{A}_{3}+\underline{A}_{4}=\underline{A} \\
& \bar{\psi}^{n+2}=\text { flux vector at time } t^{n+2}
\end{aligned}
$$

and

$$
h=t^{n+1}-t^{n}=t^{n+2}-t^{n+1}
$$

Taken separately, Eqs. (1.9a) and (1.9b) are numerically unstable unless a splitting like that of Eq. (1.7) is used in which case the alternating direction method reduces to the theta method. In order to achieve stability without the splitting of Eq. (1.7), both Eq. (1.9a) and (1.9b) must be taken together. This is accomplished by rewriting Eqs. $(1.9 a)$ and $(1.9 b)$ as

$$
\begin{aligned}
& \bar{\psi}^{n+1}=\left[I-h \underline{A}_{2}\right]^{-1}\left[\underline{I}+h \underline{A}_{1}\right] \bar{\psi}^{n} \\
& \bar{\psi}^{n+2}=\left[I-h \underline{A}_{4}\right]^{-1}\left[I+h \underline{A}_{3}\right] \bar{\psi}^{n+1}
\end{aligned}
$$

and then inserting $\mathrm{Eq} .(1.9 \mathrm{C}$ ) into $\mathrm{Eq} .(1.9 \mathrm{~d})$ :

$$
\bar{\psi}^{n+2}=\left[\underline{I}-h \underline{A}_{4}\right]^{-1}\left[\underline{I}+h \underline{A}_{3}\right]\left[\underline{I}-h \underline{A}_{2}\right]^{-1}\left[\underline{I}+h \underline{A}_{1}\right] \Psi^{n}
$$

If $\underline{A}_{1}, \underline{A}_{2}, \underline{A}_{3}$ and $\underline{A}_{4}$ are chosen properly not only can 
Eq. (1.10) be made stable, but also the matrix inversions of $\left(\underline{I}-h \underline{A}_{4}\right)$ and $\left(\underline{I}-h_{A_{2}}\right)$ can be carried out without expensive iteration.

Like other finite difference methods, alternating direction methods are characterized by a large number of unknowns. To solve the neutron diffusion equation efficientIy it is desirable to take large time steps to reduce the number of times for which these unknowns must be solved. Conceivably this may be achieved by finding an optimal matrix splitting. Alternatively, a change of variable may be used.

A successful alternating direction method, known as the MITKIN method ${ }^{12}$, uses a change of variable to achieve large time step sizes. This change of variable is known as a frequency transform. The choice of $\underline{A}_{1}, \underline{A}_{2}, \underline{A}_{3}$ and $\underline{A}_{4}$ is such that the MITKIN method is unconditionally stable. Furthermore, $\underline{A}_{2}$ and $\underline{A}_{4}$ are lower triangular matrices so that the required inversion of $\left(\underline{I}-h \underline{A}_{4}\right)$ and $\left(I-h \underline{A}_{2}\right)$ in Eq. (1.10) is comparatively easy.

\subsection{Proposed Methods}

This thesis is concerned with finite difference solutions to the neutron diffusion equation. It is assumed that the multigroup form of the neutron diffusion equation is adequate for reactor kinetics problems of interest. 
In Chapter 2 the prompt jump approximation, hitherto not applied to spatially dependent reactor kinetics problems, is applied to the theta method approach of Eq. (1.7). The assumption of this method is that the neutron flux is instantaneously in equilibrium with the delayed neutron precursors. This approximation costs little in accuracy and dampens out certain solution modes which may undesirably limit time step size.

In Chapter. 3 a recently suggested alternating direction method, the checkerboard method, is examined. The matrix splitting technique associated with the checkerboard method has both advantages and disadvantages when compared to the MITKIN matrix splitting. Also in Chapter 3 a new formulation of the frequency transform is determined. 


\section{Chapter 2 \\ THE PROMPT JUMP APPROXIMATION IN SPACE-TIME REACTOR KINETICS}

The prompt jump approximation is an approximation which may be applied to mathematical models of reactor kinetics problems. In this approximation it is assumed that the prompt and delayed neutrons of a reactor are instantaneously in equilibrium with each other.

In order to illustrate the basic idea of the prompt jump approximation, consider an initially critical reactor which is suddenly perturbed at some time $t=t_{0}$ so that it becomes subcritical. The response of the neutron population in the reactor as a function of time is shown by the solid line in Figure 2.1. The rapid initial jump of the neutron flux, $\phi(t)$, from an initial value, $\phi_{0}$, to an asymptotic behavior is called the "prompt jump".* Calculation of the detailed behavior of the flux during the jump may require many small time steps.

When the prompt jump approximation is used the detailed behavior of the flux during the jump is not calculated. Instead it is approximated as shown in Figure 2.1. Physically, this

* Some authors 13 call this the "prompt drop", reserving the name "prompt jump" for flux jumps in the positive direction. 
FIGURE 2.I

Neutron Flux Response to a Negative Step Perturbation $\phi(t)$

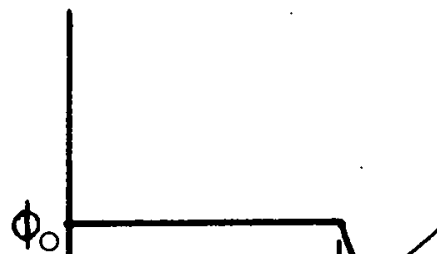
approximation to jump jump

asymptotic behavior $\log t$

approximation is equivalent to setting the prompt neutrons of the reactor into instantaneous equilibrium with the delayed neutrons. Mathematically, this approximation is equivalent to neglecting the solution mode (see Section 2.1e) associated with the jump. Because the detailed behavior of the neutron flux during the jump is not calculated, there is no necessity for small time steps to model this behavior. The prompt jump approximation is not new to nuclear reactor kinetics; it has been applied to point kinetics (space-independent kinetics) problems for many years 13,14 . Therefore, the prompt jump approximation is introduced by way of point kinetics in Section 2.1. In Section 2.2 
the prompt jump approximation is applied to the finite difference spatially-dependent neutron diffusion model. Numerical results are presented in Section 2.3 and conclusions are drawn in Section 2.4.

2.1 A Point Kinetics Explanation of the Prompt Jump The prompt jump approximation may be applied to any of the mathematical models of nuclear reactor kinetics which were described in Chapter 1: the Boltzman transport model, the diffusion theory model and the point kinetics model. Because the point kinetics model is the simplest of these, the prompt jump approximation is most easily understood when applied to this model. Therefore, the application of the prompt jump approximation to spatially-dependent kinetics will be postponed until Section 2.2. In this section point kinetics will be reviewed, the prompt jump approximation will be applied to the point kinetics model, and then the relationship of the prompt jump approximation to time step size and accuracy will be examined.

\section{2.la Review of the Point Kinetics Equations}

The equations of the point kinetics model may be derived from the space-dependent kinetics equations by integrating out all space and energy dependence of the flux. As derived in Appendix A.2, these equations are: 
$\frac{\partial \phi(t)}{\partial t}=\frac{\rho(t)-\beta}{\wedge} \phi(t)+\sum_{i=1}^{I} \lambda_{i} C_{i}(t)$.

$$
\frac{\partial C_{i}(t)}{\partial t}=\frac{\beta_{i}}{\Lambda} \phi(t)-\lambda_{i} C_{i}(t)
$$

$$
i=1,2, \cdots, I
$$

where

$$
\beta=\sum_{i=1}^{I} \beta_{i}
$$

Notation is defined in the appendix. The parameters $\wedge$ and $\boldsymbol{\beta}_{i}$ may be time dependent although such dependence is usually slight and generally can be ignored.

In matrix form, Eq. (2.1) may be written as

$$
\frac{\partial \bar{\psi}(t)}{\partial t}=\underline{R}(t) \bar{\psi}(t)
$$

where

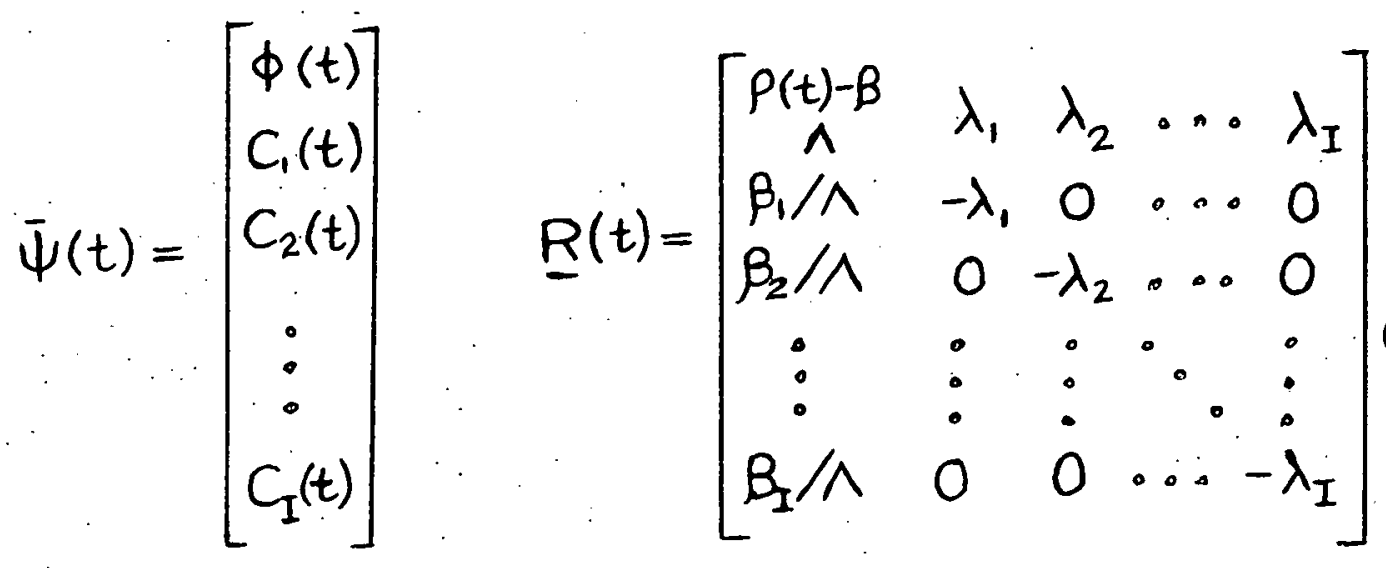


Note that Eq. (2.Id) is similar to the matrix form of the multigroup neutron diffusion equation, Eq. (1.3a), presented in Chapter 1 . The matrix $\underline{R}(t)$ is called the "point kinetics matrix". Usually the time dependence of $\underline{R}(t)$ may be neglected for small time intervals by assuming that $P(t) \approx \rho_{k}$, a constant, and $\underline{R}(t) \approx \underline{R}_{k}$, a constant matrix, over the time interval $t^{n}<t \leqslant t^{n+1}$.

2.1b Review of the Solution of the Point Kinetics Equations The solution of the point kinetics equations over the time interval $h=t^{n+1}-t^{n}$ is

$$
\begin{aligned}
& \phi\left(t^{n+1}\right)=\sum_{j=0}^{I} a_{j} e^{\omega_{j} h} \\
& C_{i}\left(t^{n+1}\right)=\sum_{j=0}^{I} \frac{\beta_{i} a_{j} e^{\omega_{j} h}}{\Lambda\left(\omega_{j}+\lambda_{i}\right)}
\end{aligned}
$$

$$
i=1,2, \cdots, I
$$

In vector-matrix form this solution may be written as

$$
\bar{\psi}\left(t^{n+1}\right)=\sum_{j=0}^{I} a_{j} e^{\omega_{j} h} \bar{\psi}_{\omega_{j}}
$$

The coefficients, $a_{j}$, are found ${ }^{15}$ to be 


$$
a_{j}=\frac{\bar{\psi}_{\omega_{j}}^{* T} \bar{\psi}\left(t^{n}\right)}{\bar{\psi}_{\omega_{j}}^{* T} \bar{\psi}_{\omega_{j}}}
$$

where $\bar{\psi}_{\omega_{j}}$ and $\bar{\psi}_{\omega_{j}}^{*}$ are the characteristic vectors (eigenvectors) of $\underline{R}_{k}$ and $\underline{R}_{k}^{T}$, respectively:

$$
\bar{\psi}_{\omega_{j}}=\left[\begin{array}{c}
1 \\
\frac{\beta_{1}}{\Lambda\left(\omega_{j}+\lambda_{1}\right)} \\
\frac{\beta_{2}}{\Lambda\left(\omega_{j}+\lambda_{2}\right)} \\
\vdots \\
\frac{\beta_{I}}{\Lambda\left(\omega_{j}+\lambda_{I}\right)}
\end{array}\right]
$$

$$
\bar{\psi}_{\omega_{j}}^{*}=\left[\begin{array}{c}
1 \\
\frac{\lambda_{1}}{\left(\omega_{j}+\lambda_{1}\right)} \\
\frac{\lambda_{2}}{\left(\omega_{j}+\lambda_{2}\right)} \\
\vdots \\
\frac{\lambda_{I}}{\left(\omega_{j}+\lambda_{I}\right)}
\end{array}\right]
$$

The $I+1$ values of $\omega_{j}$ are the roots of the "inhour formula":

$$
\rho_{k}=\Lambda \omega+\sum_{i=1}^{I} \frac{\beta_{i} \omega}{\left(\omega+\lambda_{i}\right)}
$$

This formula is the characteristic equation of $\underline{R}_{k}$; hence, each value of $\boldsymbol{\omega}$ is a characteristic value (eigenvalue) of $\underline{R}_{k}$. The parameters $\rho_{k}, \Lambda$, and $\beta_{i}$ are such that the value 
35

of each $\omega_{j}$ is real and distinct. For decay constants, $\lambda_{i}$, such that

$$
\lambda_{1}<\lambda_{2}<\because<\lambda_{I-1}<\lambda_{I}
$$

the values of $\omega_{j}$ are such that

$$
\omega_{I}<-\lambda_{I}<\omega_{I-1}<-\lambda_{I-1}<\cdots<-\lambda_{2}<\omega_{1}<-\lambda_{1}<\omega_{0} .
$$

The most positive characteristic value, $\omega_{0}$, has the same sign as $\rho_{k}$; the most negative, $\omega_{I}$, may be very large, on the order of $\omega_{I}=-100 \mathrm{sec}^{-1}$.

For a step perturbation, Eq. (2.2a) may be rewritten

as

$$
\phi(t)=\sum_{j=0}^{I} a_{j} e^{\omega_{j} t}
$$

where the change of variables $t^{n}=t^{0}=0$ and $t^{n+1}=t$ has been made. This change of variables is appropriate for step perturbation problems because the time dependent matrix, $\underline{R}(t)$, is constant for $t>t_{0}$. Therefore, $\underline{R}(t)=\underline{R}_{k}$ over the time interval $\mathrm{h}=\mathrm{t}-\mathrm{t}^{\mathrm{O}}=\mathrm{t}$. This step perturbion point kinetics flux, $\phi(t)$, may be divided into two components :

$$
\phi(t)=\phi_{a}(t)+\phi_{b}(t)
$$


where

$$
\phi_{a}(t)=\sum_{j=0}^{I-1} a_{j} e^{\omega_{j} t}
$$

and

$$
\phi_{b}(t)=a_{I} e^{\omega_{I} t}
$$

The solution component $\phi_{a}(t)$ corresponds to the asymptotic flux behavior illustrated in Figure 2.1; the solution component $\phi_{b}(t)$ corresponds to the flux jump behavior. When these two components are combined the resulting flux behavior is illustrated by the solid line in Figure 2.1.

\section{Ic The Prompt Jump Approximation Applied to the Point} Kinetics Model

The prompt jump approximation is applied to the point kinetics model by assuming that the prompt neutrons of the model are instantaneously in equilibrium with the delayed neutrons. Mathematically, this is equivalent to setting the neutron flux directly proportional to the delayed neutron precursor concentrations. This may be achieved by neglecting the time derivative of the flux, $\frac{\partial \phi(t)}{\partial t}$ in Eq. (2.1a). Hence the prompt jump point kinetics equations are the following approximation to the point kinetics equations : 


$$
\begin{aligned}
& 0=\frac{\rho(t)-\beta}{\Lambda} \phi(t)+\sum_{i=1}^{I} \lambda_{i} C_{i}(t) \\
& \frac{\partial C_{i}(t)}{\partial t}=\frac{\beta_{i}}{\Lambda} \phi(t)-\lambda_{i} C_{i}(t) \quad i=1,2 ; \cdots, I
\end{aligned}
$$

When Eq. (2.5a) is rewritten as

$$
\phi(t)=\frac{\Lambda}{\beta-\rho(t)} \sum_{i=1}^{I} \lambda_{i} C_{i}(t)
$$

it is seen that the neutron flux is directly proportional to the delayed neutron precursors. It is also seen from Eq. (2.5c) that $\phi(t)$ will be negative if $\rho(t)>\beta$. Therefore the prompt jump approximation is invalid for prompt supercritical transients and is poor for problems where $\rho(t) \rightarrow \beta$.

The matrix form of the prompt jump point kinetics equations is:

$$
\frac{\partial \bar{C}(t)}{\partial t}=\underline{R}^{\prime}(t) \bar{C}(t)
$$


where

$$
\bar{C}(t)=\left[\begin{array}{c}
C_{1}(t) \\
C_{2}(t) \\
0 \\
0 \\
C_{I}(t)
\end{array}\right] ; \underline{R}^{\prime}(t)=\left[\begin{array}{cccc}
\frac{\beta_{1} \lambda_{1}}{\beta-\rho(t)} & \frac{\beta_{1} \lambda_{2}}{\beta-\rho(t)} & \cdots & \frac{\beta_{1} \lambda_{I}}{\beta-\rho(t)} \\
\frac{\beta_{2} \lambda_{1}}{\beta-\rho(t)} & \frac{\beta_{2} \lambda_{2}}{\beta-\rho(t)} & \cdots & \frac{\beta_{2} \lambda_{I}}{\beta-\rho(t)} \\
\cdot & \ddots & 0 \\
\frac{\beta_{I} \lambda_{1}}{\beta-\rho(t)} & \frac{\beta_{I} \lambda_{2}}{\beta-\rho(t)} & \cdots & \frac{\beta_{I} \lambda_{I}}{\beta-\rho(t)}
\end{array}\right]
$$

The time dependence of $\underline{R}^{\prime}(t)$ may be neglected over small time intervals, $h=t^{n+l}-t^{n}$, by assuming that $\rho(t) \approx \rho_{k}$ and $\underline{R}^{\prime}(t) \approx \underline{R}_{k}^{\prime}$, a constant matrix, over the interval $h$.

\section{Id The Solution of the Prompt Jump Point Kinetics}

\section{Equations}

The solution of the prompt jump point kinetics equations over the time interval $h=t^{n+l}-t^{n}$ is

$$
\phi\left(t^{n+1}\right)=\frac{\Lambda}{\beta-\rho_{k}} \sum_{i=1}^{I} \lambda_{i} C_{i}\left(t^{n+1}\right)
$$

where

$$
C_{i}\left(t^{n+1}\right)=\sum_{j=0}^{I-1} \frac{\beta_{i} a_{j}^{\prime} e^{\omega_{j}^{\prime} h}}{\Lambda\left(\omega_{j}^{\prime}+\lambda_{i}\right)} \quad \begin{aligned}
& \\
& i=1,2, \cdots, I
\end{aligned}
$$


This solution may be expressed in vector-matrix form as

$$
\bar{C}\left(t^{n+1}\right)=\sum_{j=0}^{I-1} a_{j}^{\prime} e^{\omega_{j}^{\prime} h} \bar{C}_{\omega_{j}^{\prime}}
$$

This is seen to be the solution of Eq. (2.5d) where $\underline{R}^{\prime}(t)$ has been replaced by $\underline{R}_{k}$ over the time interval $h=t^{n+1}-t^{n}$. The coefficients, $a_{j}^{\prime}$, in this solution are found in an analogous fashion to the coefficients, $\boldsymbol{a}_{\mathbf{j}}$, of the point kinetics equations:

$$
a_{j}^{\prime}=\frac{\bar{C}_{\omega_{j}^{\prime}}^{*} \bar{C}\left(t^{*}\right)}{\bar{C}_{\omega_{j}^{\prime}}^{* \top} \bar{C} \omega_{j}^{\prime}}
$$

The I values of the coefficients $a_{j}^{\prime}$ computed from Eq. (2.6d) are usually very close in value to the first I values of the coefficients $a_{j}$ computed from Eq. (2.2d). The vectors, $\bar{C}_{\omega_{j}^{\prime}}$ and $\bar{C}_{\omega_{j}^{\prime}}^{*}$ are the characteristic vectors of $\underline{R}^{\prime} k$ and $R^{\prime T} \frac{T}{k}$, respectively:

$$
\bar{C}_{\omega_{j}^{\prime}}=\left[\begin{array}{c}
\frac{\beta_{1}}{\Lambda\left(\omega_{j}^{\prime}+\lambda_{1}\right)} \\
\frac{\beta_{2}}{\Lambda\left(\omega_{j}^{\prime}+\lambda_{2}\right)} \\
\vdots \\
\frac{\beta_{I}}{\Lambda\left(\omega_{j}^{\prime}+\lambda_{I}\right)}
\end{array}\right] \quad ; \quad \bar{C}_{\omega_{j}^{\prime}}^{*}=\left[\begin{array}{c}
\frac{\lambda_{1}}{\left(\omega_{j}^{\prime}+\lambda_{1}\right)} \\
\frac{\lambda_{2}}{\left(\omega_{j}^{\prime}+\lambda_{2}\right)} \\
\vdots \\
\frac{\lambda_{I}}{\left(\omega_{j}^{\prime}+\lambda_{I}\right)}
\end{array}\right]
$$


Note that the parameter $\Lambda$, which is not present in the matrix $\underline{R}_{k}^{\prime}$, appears in Eq. (2.6b) and in the vector $\bar{C}_{\omega_{j}^{\prime}}$ in order to make $\bar{C}_{\omega_{j}^{\prime}}$ dimensionless.

The I values of $\omega_{j}^{\prime}$ are the real and distinct roots of the equation

$$
\rho_{k}=\sum_{i=1}^{I} \frac{\beta_{i} \omega^{\prime}}{\left(\omega^{\prime}+\lambda_{i}\right)}
$$

This formula is the characteristic equation of $\underline{R}_{k}$, so each value of $\omega_{j}$ is a characteristic value of $\underline{R}_{k}$. Generally $\Lambda$ is a very small number; hence Eq. (2.7) is a good approximation to Eq. (2.3) and the I roots, $\omega_{j}^{\prime}$, of Eq. (2.7) are almost identical with the first I roots, $\omega_{j}$, of Eq. (2.3). The characteristic values $\omega_{j}^{\prime}$ and $\omega_{j}$ are plotted in Figure 2.2 as functions of $\rho_{k}$. As is illustrated in Figure 2.2, the most negative root of Eq. (2.7) has the magnitude

$$
\left|\omega_{I-1}^{\prime}\right|<\lambda_{I}
$$

Because $\lambda_{I}$ is less than $4 \mathrm{sec}^{-1}$ for most applications, this most negative root of the prompt jump solution is much smaller than the most negative root, $\left|\omega_{I}\right|$, of the point kinetics solution which can be on the order of $100 \mathrm{sec}^{-1}$. 
FIGURE 2.2
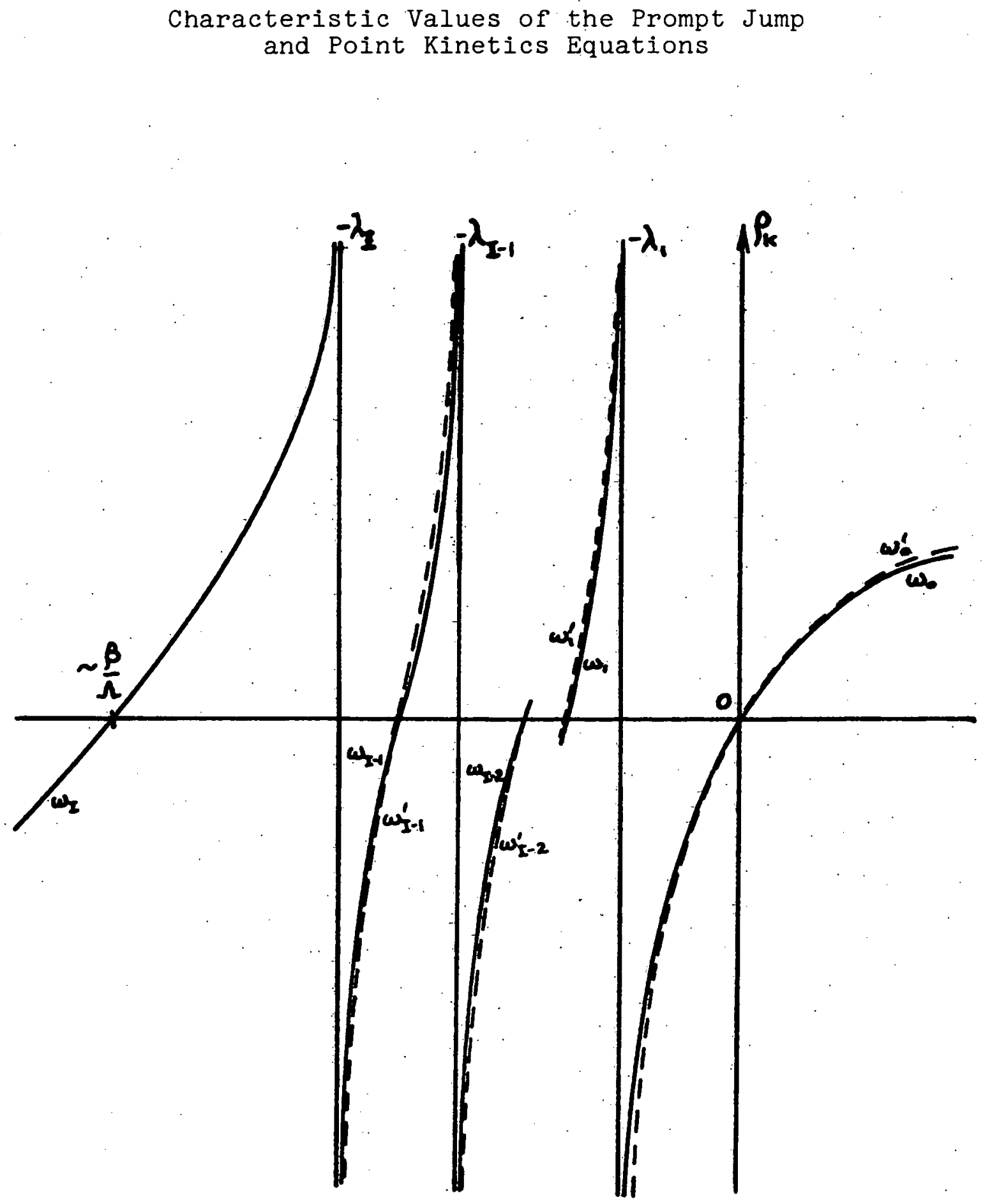
The prompt jump flux is found by combining Eqs.

$(2.6 a),(2.6 b)$ and $(2.7)$.

$$
\begin{aligned}
\phi\left(t^{n+1}\right) & =\frac{\Lambda}{\beta-\rho_{k}} \sum_{i=1}^{I} \lambda_{i} C_{i}\left(t^{n+1}\right) \\
& =\frac{\Lambda}{\beta-\rho_{k}} \sum_{i=1}^{I} \lambda_{i} \sum_{j=0}^{I-1} \frac{\beta_{i} a_{j}^{\prime} e^{\omega_{j}^{\prime} h}}{\Lambda\left(\omega_{j}^{\prime}+\lambda_{i}\right)} \\
& =\frac{1}{\beta-\rho_{k}} \sum_{j=0}^{I-1}\left[\sum_{i=1}^{I} \frac{\beta_{i} \lambda_{i}}{\left(\omega_{j}^{\prime}+\lambda_{i}\right)}\right] a_{j}^{\prime} e^{\omega_{j}^{\prime} h} \\
& =\frac{1}{\beta-\rho_{k}} \sum_{j=0}^{I-1}\left[B-\rho_{k}\right] a_{j}^{\prime} e^{\omega_{j}^{\prime} h} \\
& =\sum_{j=0}^{I-1} a_{j}^{\prime} e^{\omega_{j}^{\prime} h}
\end{aligned}
$$

For a step perturbation; Eq.'(2.8) may be rewritten

as

$$
\phi_{p}(t)=\sum_{j=0}^{I-1} a_{j}^{\prime} e^{\omega_{j}^{\prime} t}
$$

where the change of variables, $t^{n}=t^{0}=0$ and $t^{n+l}=t$ has been made. This change of variables is appropriate because $\underline{R}^{\prime}(t)=\underline{R}_{k}^{\prime}$ over the time interval $h=t-t^{0}=t$ 
for step perturbations. Note that the prompt jump point kinetics flux, $\phi_{p}(t)$, is here distinguished from the point kinetics flux, $\phi(t)$, by the subscript $p$.

Because the I values of $a_{j}^{\prime}$ and $w_{j}^{\prime}$ in Eq. (2.9a) are very close in value to the first $I$ values of $a_{j}$ and $\omega_{j}^{\prime}$ in Eq. (2.4a),

$$
\sum_{j=0}^{I-1} a_{j}^{\prime} e^{\omega_{j}^{\prime} t} \approx \sum_{j=0}^{I-1} a_{j} e^{\omega_{j} t}
$$

Thus from Eqs. (2.4c) and (2.9b),

$$
\phi_{p}(t) \approx \phi_{a}(t)
$$

That is, the prompt jump point kinetics flux is almost identical to the asymptotic component of the point kinetics flux for step perturbations. Hence the prompt jump approximation accurately models the asymptotic behavior of the flux (see Figure 2.1.) but does not model the flux jump illustrated by the solid line of Figure 2.1. Instead the prompt jump approximation models the neutron flux of negative step perturbations as shown by the broken line of Figure 2.1.

2. Ie The Effect of the Prompt Jump Approximation on Time Step Size - - A Point Kinetics Argument

In order to determine a relationship between the prompt jump approximation and time step size it is necessary to discretize the kinetics equations. Because the point kinetics equations are the simplest kinetics equations it 
is useful to consider the solution of the discretized point kinetics and prompt jump point kinetics equations.

The point kinetics equation,

$$
\frac{\partial \bar{\psi}}{\partial t}(t)=\underline{R}(t) \bar{\psi}(t)
$$

may be discretized as

$$
\frac{\bar{\psi}\left(t^{n+1}\right)-\bar{\psi}\left(t^{n}\right)}{h}=\theta \underline{R}_{k} \bar{\psi}\left(t^{n+1}\right)+(1-\theta) \underline{R}_{K} \bar{\psi}\left(t^{n}\right)
$$

where $\theta$ is a number such that $0 \leq \theta \leq 1$

This discretization is similar to that of the theta method which was described in Section 1.4a. Eq. (2.10a) may be further rearranged as

$$
\bar{\psi}\left(t^{n+1}\right)=\left[\underline{I}-h \theta \underline{R}_{k}\right]^{-1}\left[\underline{I}+h(1-\theta) \underline{R}_{k}\right] \bar{\psi}\left(t^{n}\right)
$$

and by use of the expansion*

$$
\bar{\psi}\left(t^{n}\right)=\sum_{j=0}^{I} a_{j} \bar{\psi}_{w_{j}}
$$

* The matrix $\underline{R}_{k}$ has a complete set of characteristic vectors and distinct characteristic values. Therefore, $\dot{\psi}\left(t^{\prime \prime}\right)$ may be expanded as in Eq. (2.10c). This expansion may also be deduced from Eq. $(2.2 \mathrm{c})$. 
and Sylvester's Theorem*, it can be shown that Eq. (2.10b) is equivalent to.

$$
\bar{\psi}\left(t^{n+1}\right)=\sum_{j=0}^{I} a_{j}\left[1-h \theta \omega_{j}\right]^{-1}\left[1+h(1-\theta) \omega_{j}\right] \bar{\psi}_{\omega_{j}}
$$

The discretized point kinetics flux is simply the first element of this vector:

$$
\phi_{d}\left(t^{n+1}\right)=\sum_{j=0}^{I} a_{j}\left[1-h \theta \omega_{j}\right]^{-1}\left[1+h(1-\theta) \omega_{j}\right]
$$

The subscript d has been added to denote that $\phi_{d}\left(t^{n+i}\right)$ is the solution of the discretized point kinetics equations. Note that

$$
\left[1-h \theta \omega_{j}\right]^{-1}\left[1+h(1-\theta) \omega_{j}\right] \approx e^{\omega_{j} h} \text { as } \quad\left|\omega_{j} h\right| \rightarrow \operatorname{small}
$$

Thus $\phi_{d}\left(t^{n+1}\right)$ approaches the true solution,

$$
\phi\left(t^{n+1}\right)=\sum_{j=0}^{I} a_{j} e^{\omega_{j h}}
$$

as $\left|\omega_{j} h\right|$ becomes small. Obviously if $\left|\omega_{j}\right|$ is large and $\left|\omega_{j} h\right|$ is required to be small, $h$ must be very small. In Section $2.1 \mathrm{~b}$ it was shown that the largest characteristic value of the point kinetics equation, $\omega_{I}$, is large.

* Sylvester's Theorem ${ }^{16}$ states that

$$
f(\underline{M}) \cdot \bar{x}_{j}=f\left(\lambda_{j}\right) \cdot \bar{x}_{j} . \quad j=1,2, \ldots, n
$$

where $\mathrm{M}=$ any $\mathrm{nxn}$ matrix with distinct characteristic values and characteristic vectors;

$\bar{x}_{j}=j$ th distinct characteristic vector of $\underline{M}$;

$\begin{aligned} \mathrm{A}_{\mathrm{j}} & =j \text { th distinct charact } \\ & =\text { any function of } \mathrm{M} \text {; }\end{aligned}$

$f\left(\bar{\lambda}_{j}\right)=$ the same function of $\lambda_{j}$ 
Therefore, $h$ must be very small for the solution of the discretized point kinetics equations to accurately approximate the true solution.

The prompt jump point kinetics equation, Eq. (2.5d), may also be discretized similarly to the theta method of Section 1.4a:

$$
\frac{\bar{C}\left(t^{n+1}\right)-\bar{C}\left(t^{n}\right)}{h}=\theta \underline{R}_{k}^{\prime} \bar{C}\left(t^{n+1}\right)+(1-\theta) \underline{R}_{k}^{\prime} \bar{C}\left(t^{n}\right)
$$

This may be rearranged as

$$
\bar{C}_{p d}\left(t^{n+1}\right)=\left[\underline{I}-h \theta \underline{R}_{k}^{\prime}\right]^{-1}\left[\underline{I}+h(1-\theta) \underline{R}_{k}^{\prime}\right] \bar{C}\left(t^{n}\right)
$$

The subscript pd has been added to denote that $\bar{C}_{p d}\left(t^{n+1}\right)$ is determined from the prompt jump discretized equations. The vector $\bar{C}\left(t^{n}\right)$ may be expanded as

$$
\bar{C}\left(t^{n}\right)=\sum_{j=0}^{I-1} a_{j}^{\prime} \bar{C}_{w_{j}^{\prime}}
$$

and Sylvester's Theorem may be applied to yield

$$
\bar{C}_{p d}\left(t^{n+1}\right)=\sum_{j=0}^{I-1} a_{j}^{\prime}\left[1-h \theta \omega_{j}^{\prime}\right]^{-1}\left[1+h(1-\theta) \omega_{j}^{\prime}\right] \bar{C}_{\omega_{j}^{\prime}}
$$

The I elements of this vector are

$$
c_{i p d}\left(t^{n+1}\right)=\sum_{j=0}^{I-1} a_{j}^{\prime}\left[1-h \theta \omega_{j}^{\prime}\right]^{-1}\left[1+h(1-\theta) \omega_{j}^{\prime}\right] \frac{\dot{B}_{i}}{\wedge\left(\omega_{j}^{\prime}+\lambda_{i}\right)}
$$


The discretized prompt jump point kinetics flux may now be determined from Eq. (2.12) by an approach similar to that of Eq. (2.8)

$$
\begin{aligned}
\phi_{p d}\left(t^{n+1}\right) & =\frac{\Lambda}{\beta-\rho_{k}} \sum_{i=1}^{I} \lambda_{i} C_{i p d}\left(t^{n+1}\right) \\
& =\frac{1}{\beta-\rho_{k}} \sum_{j=0}^{I-1}\left[\sum_{i=1}^{I} \frac{\beta_{i} \lambda_{i}}{\left(\omega_{j}^{\prime}+\lambda_{i}\right)}\right] a_{j}^{\prime}\left[1-h \theta \omega_{j}^{j}\right]^{-1}\left[1+h(1-\theta) \omega_{j}^{\prime}\right] \\
& =\sum_{j=0}^{I-1} a_{j}^{\prime}\left[1-h \theta \omega_{j}^{\prime}\right]^{-1}\left[1+h(1-\theta) \omega_{j}^{\prime}\right]
\end{aligned}
$$

As $\left|\omega_{j}^{\prime} h\right|$ becomes small,

$$
\phi_{p d}\left(t^{n+1}\right) \rightarrow \sum_{j=0}^{I-1} a_{j}^{\prime} e^{\omega_{j}^{\prime} h}
$$

Thus the degree to which $\phi_{p d}\left(t^{n+1}\right)$ approximates the true prompt jump solution,

$$
\phi_{p}\left(t^{n+1}\right)=\sum_{j=0}^{I-1} a_{j}^{\prime} e^{\omega_{j}^{\prime} h}
$$

depends upon how small the $\omega_{j}^{\prime} h$ terms are. Obviously, if $\left|\omega_{j}^{\prime}\right|$ is smali, then $h$ need not be small for $\left|\omega_{j}^{\prime} h\right|$ to be small. 
In Section 2.1d it was stated that the largest characteristic value, $\omega_{I}$, of the point kinetics equations is much larger in magnitude than the largest characteristic value, $\omega_{I-1}^{\prime}$, of the prompt jump point kinetics equations. Because $\left|\omega_{I-1}^{\prime}\right| \ll\left|\omega_{I}\right|$, the discretized prompt jump flux, $\phi_{p d}\left(t^{n+1}\right)$, more closely approximates. the true prompt jump flux, $\phi_{p}\left(t^{n+1}\right)$, than the discretized point kinetics flux, $\phi_{d}\left(t^{n+1}\right)$, approximates the true point kinetics flux, $\phi\left(t^{n+1}\right)$, for a given time step size, $h$. Alternatively stated, a larger time step size, $h$, may be used to approximate $\phi_{p}\left(t^{n+1}\right)$ by $\phi_{p d}\left(t^{n+1}\right)$ than may be used to approximate $\phi\left(t^{n+1}\right)$ by $\phi_{d}\left(t^{n+1}\right)$ for a given accuracy.

If it is not necessary to model the detailed flux jump behavior illustrated in Figure 2.1 then the prompt jump approximation is useful. Furthermore, larger time step sizes may be used in the discretized equations. Therefore, larger time step sizes may be made possible by the application of the prompt jump approximation. 2.1f The Two-Level Kinetics Matrix Discretization

Before applying the prompt jump approximation to spatially dependent kinetics it is useful to examine one more variation of the point kinetics equations.

Let the discretized point kinetics equation, Eq. (2.10a), be modified as 


$$
\frac{\bar{\psi}\left(t^{n+1}\right)-\bar{\psi}\left(t^{n}\right)}{h}=\theta \underline{R}_{n+1} \bar{\psi}\left(t^{n+1}\right)+(1-\theta) \underline{R}_{n} \bar{\psi}\left(t^{n}\right)
$$

where $\quad \underline{R}_{n+1}=\underline{R}\left(t^{n+1}\right)$

$$
\underline{R}_{n}=\underline{R}\left(t^{n}\right)
$$

Because the point kinetics nuclear parameters in the matrix are evaluated at two levels in time, this discretization may be called the two-level kinetics matrix discretization. The discretization of Eq. (2.10a) may be called a single-level kinetics matrix discretization because $\underline{R}(t)$ is evaluated at a single time level, $t^{k}$, where $t^{n} \leqslant t^{k} \leqslant t^{n+1}$. The two-level discretization of Eq. (2.14) has two important consequences.

First, results are offset in time for step perturbations. Suppose a reactor is perturbed at some time $t=t^{n}$. Then the correct discretized formulation of the point kinetics equations is

$$
\frac{\bar{\psi}\left(t^{n+1}\right)-\bar{\psi}\left(t^{n}\right)}{h}=\theta \underline{R}_{n+1} \bar{\psi}\left(t^{n+1}\right)+(1-\theta) \underline{\underline{R}}_{n+1} \bar{\psi}\left(t^{n}\right)
$$

That is, immediately after the perturbation, $\underline{R}(t)$ assumes the constant value $\underline{R}_{n+1}$ throughout the time interval $h=t^{n+1}-t^{n}$. $\underline{R}(t)$ is not an average of $\underline{R}_{n}$ and $\underline{R}_{n+1}$ as is implied by the two-level discretization. Now if $\theta=1$, both Eqs. (2.14) and. (2.15) become identical. But if 
$\theta=0$, then. $\bar{\psi}\left(t^{n+1}\right)$ of Eq. (2.14) equals $\bar{\psi}\left(t^{n}\right)$ of Eq. (2.15). In general, the solution of the two-level equation lags behind the solution of the single-level equation a according to the relationship

$\bar{\Psi}(t+h)$

(two-level formulation)

$$
=\bar{\psi}(t+\theta h)
$$

(single-level formulation)

As a result of this time lag effect, which is important for large values of $h$, the two-level discretization overpredicts the solution for negative step perturbations and underpredicts the solution for positive step perturbations.

The second consequence of the two-level kinetics matrix discretization is that the solution of Eq. (2.14) more closely resembles the discretized prompt. jump point kinetics solution than it resembles the single level discretization point kinetics solution for certain choices of $\theta$. This can be illustrated by a step perturbation example with $\theta=\frac{1}{2}$.

Suppose a critical reactor is perturbed at some time $t^{n}$. Then let

$$
\begin{aligned}
& \underline{R}(t)=\underline{R}_{0}=\underline{R}_{n} \quad \text { for } \quad t \leqslant t^{n} \\
& \underline{R}(t)=\underline{R}_{k}=\underline{R}_{n+1} \quad \text { for } \quad t>t^{n}
\end{aligned}
$$

Note that $\underline{R}_{0} \bar{\psi}\left(t^{n}\right)=0$ from the initial steady state criticality condition, 
$\frac{\partial \bar{\psi}(t)}{\partial t}=\underline{R}(t) \bar{\psi}(t)=0 \quad$ for $t \leqslant t^{n}$

The solution of Eq. (2.14) for this problem is found by rearranging Eq. $(2.14)$ as

$$
\begin{aligned}
\bar{\psi}\left(t^{n+1}\right) & =\left[\underline{I}-h \theta \underline{R}_{n+1}\right]^{-1}\left[\underline{I}+h(1-\theta) \underline{R}_{n}\right] \bar{\psi}\left(t^{n}\right) \\
& =\left[\underline{I}-\frac{1}{2} h \underline{R}_{K}\right]^{-1}\left[\underline{I}+\frac{1}{2} h \underline{R}_{0}\right] \bar{\psi}\left(t^{n}\right) \\
& =\left[\underline{I}-\frac{1}{2} h \underline{R}_{K}\right]^{-1}\left[\bar{\psi}\left(t^{n}\right)+\frac{1}{2} h \underline{R}_{0} \bar{\psi}\left(t^{n}\right)\right] \\
& =\left[\underline{I}-\frac{1}{2} h \underline{R}_{K}\right]^{-1} \bar{\psi}\left(t^{n}\right)
\end{aligned}
$$

Sylvester's Theorem and the expansion

$$
\bar{\psi}\left(t^{n}\right)=\sum_{j=0}^{I} a_{j} \bar{\psi}_{\omega_{j}}
$$

may be used to give

$$
\bar{\psi}\left(t^{n+1}\right)=\sum_{j=0}^{I} a_{j}\left(1-\frac{1}{2} \omega_{j} h\right)^{-1} \bar{\psi}_{\omega_{j}}
$$

The first element of this vector, $\phi_{2 d}\left(t^{n+1}\right)$, is the flux of the two-level discretized point kinetics equations: 
52

$$
\phi_{2 d}\left(t^{n+1}\right)=\sum_{j=0}^{I} a_{j}\left(1-\frac{1}{2} \omega_{j} h\right)^{-1}
$$

This is to be compared with the prompt jump point kinetics flux found from Eq. (2.13)

$$
\Phi_{p d}\left(t^{n+1}\right)=\sum_{j=0}^{I-1} a_{j}^{\prime}\left(1-\frac{1}{2} \omega_{j}^{\prime} h\right)^{-1}\left(1+\frac{1}{2} \omega_{j}^{\prime} h\right)
$$

and with the single-level discretization point kinetics flux found from Eq. (2.11)

$$
\begin{aligned}
& \phi_{d}\left(t^{n+1}\right)=\sum_{j=0}^{I} a_{j}\left(1-\frac{1}{2} \omega_{j} h\right)^{-1}\left(1+\frac{1}{2} \omega_{j} h\right) \\
& \text { In Section 2.1b it was shown that } \\
& \left|\omega_{I}\right|>>\left|\omega_{j}\right| \quad j=0,1, \cdots, I-1
\end{aligned}
$$

For the sake of this example assume that $h$ is such that

$$
\left|\omega_{I} h\right| \gg 1>\left|\omega_{j} h\right| \quad j=0,1, \ldots, I-1
$$

Then

$$
\begin{aligned}
\phi_{2 d}\left(t^{n+1}\right) & =\sum_{j=0}^{I} a_{j}\left(1-\frac{1}{2} w_{j} h\right)^{-1} \\
& =a_{I}\left(1-\frac{1}{2} \omega_{I} h\right)^{-1}+\sum_{j=0}^{I-1} a_{j}\left(1-\frac{1}{2} \omega_{j} h\right)^{-1}
\end{aligned}
$$


53

$$
\begin{aligned}
& \approx a_{I}\left(-\frac{1}{2} \omega_{I} h\right)^{-1}+\sum_{j=0}^{I-1} a_{j} \\
& \approx \sum_{j=0}^{I-1} a_{j}
\end{aligned}
$$

This approximates the prompt jump solution,

$$
\begin{aligned}
\phi_{p d}\left(t^{n+1}\right) & =\sum_{j=0}^{I-1} a_{j}^{\prime}\left(1-\frac{1}{2} \omega_{j}^{\prime} h\right)^{-1}\left(1+\frac{1}{2} \omega_{j}^{\prime} h\right) \\
& \approx \sum_{j=0}^{I-1} a_{j}^{\prime} \\
& \approx \sum_{j=0}^{I-1} a_{j}
\end{aligned}
$$

However, the single-level point kinetics discretization has a different solution:

$$
\begin{aligned}
\phi_{d}\left(t^{n+1}\right) & =\sum_{j=0}^{I} a_{j}\left(1-\frac{1}{2} \omega_{j} h\right)^{-1}\left(1+\frac{1}{2} \omega_{j} h\right) \\
& =a_{I}\left(1-\frac{1}{2} \omega_{I} h\right)^{-1}\left(1+\frac{1}{2} \omega_{I} h\right)+\sum_{j=0}^{I-1} a_{j}\left(1-\frac{1}{2} \omega_{j} h\right)^{-1}\left(1+\frac{1}{2} \omega_{j} h\right) \\
& \approx a_{I}\left(-\frac{1}{2} \omega_{I} h\right)^{-1}\left(\frac{1}{2} \omega_{I} h\right)+\sum_{j=0}^{I-1} a_{j} \\
& \approx-a_{I}+\sum_{j=0}^{I-1} a_{j}
\end{aligned}
$$


This is erroneous because the Itlst.component of the true point kinetics solution, $a_{I} e^{w_{I} h}$, cannot be represented by

$$
a_{I}\left(1-\frac{1}{2} w_{I} h\right)^{-1}\left(1+\frac{1}{2} w_{I} h\right) \approx-a_{I}
$$

when $\left|w_{1} h\right|$ is large. But because

$$
a_{I} e^{\omega_{I} h} \approx 0 \quad \text { for } \omega_{I} h \ll-1
$$

the two-level representation,

$$
a_{I}\left(1-\frac{1}{2} \omega_{I} h\right)^{-i} \approx 0 \quad\left|\omega_{I} h\right|>>1
$$

is acceptable.

For the solution at later time steps, $\bar{\psi}\left(t^{n+2}\right), \bar{\psi}\left(t^{n+3}\right), \cdots, \bar{\psi}\left(t^{n+l}\right)$, both the single-level and two-level formulations are the same :

$$
\frac{\bar{\psi}\left(t^{n+l+1}\right)-\bar{\psi}\left(t^{n+l}\right)}{h}=\theta \underline{R}_{k} \bar{\psi}\left(t^{n+l+1}\right)+(1-\theta) \underline{R}_{k} \bar{\Psi}\left(t^{n+l}\right)
$$

However, both formulations depend upon the accuracy of $\phi\left(t^{n+1}\right)$ and hence better accuracy is expected from the two-level kinetics matrix discretization.

The crude example of this section illustrates that both the two-level discretization and the prompt jump approxi- 
mation avoid the troublesome I+lst solution mode, $a_{I} e^{\omega_{I} h}$, of the point kinetics solution for at least one specific situation. However, it should not be inferred that these two methods are equivalent just because both methods may seem better than the single-level discretization method. Indeed, these methods are all quite dissimilar. In order to assess truly the usefuiness of the prompt jump approximation, particularly in the case of spatially dependent problems, comparison of numerical results is necessary: This will be done in the following sections.

\subsection{The Spatially Dependent Prompt Jump Approximation}

The prompt jump approximation may be applied to spatially dependent mathematical: models of reactor kinetics by setting the prompt neutrons of the model instantaneously into equilibrium with the delayed neutrons of the model. Although this approximation is applicable to the transport theory model, only the simpler diffusion model applícation will be considered. The solution technique will be that of the relatively uncomplicated theta method described in Section 1.4a. Only one spatial dimension and one energy group problems will be examined so that the matrices to be inverted when the theta method is applied are tridiagonal.

In Section 2.2a a one-group, one-dimensional finite difference formulation of the neutron diffusion equation will be devised. The prompt jump approximation to these equations will be determined in Section 2.2b. Comparison 
of these methods will be presented in Section 2.3.

2.2a A One-Group, One-Dimensional Theta Method Neutron Diffusion Equation

The one-group, one-dimensional neutron diffusion equation as derived in Appendix A. $3 a$ is

$$
\begin{aligned}
\frac{1}{v} \frac{\partial}{\partial t} \phi(x, t)=\frac{\partial}{\partial x} D(x, t) \frac{\partial}{\partial x} \phi(x, t)-\Sigma_{a}(x, t) \phi(x, t) \\
\quad+(1-\beta) \frac{\nu \Sigma_{f}(x, t)}{k_{e f f}} \phi(x, t)+\sum_{i=1}^{I} \lambda_{i} C_{i}(x, t)
\end{aligned}
$$

The delayed neutron precursor concentrations are found by

$$
\frac{\partial C_{i}(x, t)}{\partial t}=\beta_{i} \frac{\nu \Sigma_{f}(x, t)}{k_{e f f}} \phi(x, t)-\lambda_{i} C_{i}(x, t) \quad i \quad i=1,2, \ldots, I
$$

where

$$
\begin{aligned}
\phi(x, t)= & \text { neutron flux } \\
C_{i}(x, t)= & \text { precursor concentration for ith } \\
& \text { precursor group } \\
D(x, t)= & \text { diffusion constant } \\
\Sigma_{a}(x, t)= & \text { macroscopic absorption cross section } \\
K_{e f f}= & \text { multiplication factor } \\
\nu \Sigma_{f}(x, t)= & \text { fission production cross section } \\
\beta_{i}= & \text { delayed neutron fraction for ith } \\
& \text { precursor group }
\end{aligned}
$$




$$
\begin{aligned}
& \beta=\sum_{i=1}^{I} \beta_{i} \\
& \lambda_{i}=\text { decay constant for ith precursor group }
\end{aligned}
$$

Eqs. (2.16) may be simplified by introducing the operators

$$
\begin{aligned}
& G(x, t) \equiv \frac{\partial}{\partial x} D(x, t) \frac{\partial}{\partial x}-\Sigma_{a}(x, t) \\
& F(x, t) \equiv \frac{\nu \Sigma_{f}(x, t)}{k_{e f f}}
\end{aligned}
$$

Then insertion of these operators into Eqs. (2.16) leads to

$$
\begin{aligned}
& \frac{1}{v} \frac{\partial}{\partial t} \phi(x, t)= G(x, t) \phi(x, t)+(1-\beta) F(x, t) \phi(x, t) \\
& \quad+\sum_{i=1}^{I} \lambda_{i} C_{i}(x, t) \\
& \frac{\partial C_{i}(x, t)=}{\partial t}=\beta_{i} F(x, t) \phi(x, t)-\lambda_{i} C_{i}(x, t) \\
& i=1,2, \cdots, I
\end{aligned}
$$

In finite difference form the diffusion operator, $G(x, t)$, may be represented by a three point central difference formula. Thus $G(x, t) \phi(x, t)$ and $F(x, t) \phi(x, t)$ may be evaluated at each spatial mesh point, $x_{m}$, and temporal mesh point, $t^{n}$; as 


$$
\begin{aligned}
& \left.G\left(x, t^{n}\right) \phi\left(x, t^{n}\right)\right|_{x=x_{m}} \equiv G_{m}^{n} \phi_{m}^{n} \\
& =\frac{2}{h_{x_{m}}+h_{x_{m-1}}}\left[\frac{D_{m}^{n}\left(\phi_{m+1}^{n}-\phi_{m}^{n}\right)}{h_{x_{m}}}-\frac{D_{m-1}^{n}\left(\phi_{m}^{n}-\phi_{m-1}^{n}\right)}{h_{x_{m-1}}}\right]-\frac{1}{2}\left[\sum_{a_{m}}^{n}+\sum_{a_{m-1}}^{n}\right] \phi_{m}^{n} \\
& \left.F\left(x, t^{n}\right) \phi\left(x, t^{n}\right)\right|_{x=x_{m}} \equiv F_{m}^{n} \phi_{m}^{n}=\frac{1}{2 k_{e f f}}\left[\nu \sum_{f_{m}}^{n}+\nu \sum_{f_{m-1}}^{n}\right] \phi_{m}^{n}
\end{aligned}
$$

The notation of Eq. (2.17) is such that for $x_{m-1}<x<x_{m}$ the nuclear parameters $D\left(x, t^{n}\right), \Sigma_{a}\left(x, t^{n}\right)$ and $\nu \Sigma_{f}\left(x, t^{n}\right)$ have the values $D_{m-1}^{n}, \sum_{a m-1}^{n}$ and $\nu \sum_{f m-1}^{n}$; for $x_{m}<x<x_{m+1}$ these nuclear parameters are $D_{m}^{n}, \Sigma_{a_{m}}^{n}$ and $\nu \Sigma_{f_{m}}^{n}$. Also, $h_{x_{m-1}} \equiv x_{m}-x_{m-1}$ and $h_{x_{m}} \equiv x_{m+1}-x_{m}$ These definitions lead to the following finite difference theta method form of the neutron diffusion equation:

$$
\begin{aligned}
\frac{\phi_{m}^{n+1}-\phi_{m}^{n}}{v h} & =\theta_{p}\left[G_{m}^{n+1} \phi_{m}^{n+1}+(1-\beta) F_{m}^{n+1} \phi_{m}^{n+1}\right] \\
& +\left(1-\theta_{p}\right)\left[G_{m}^{n} \phi_{m}^{n}+(1-\beta) F_{m}^{n} \phi_{m}^{n}\right]+\sum_{i=1}^{I} \lambda_{i}\left[\theta_{D} C_{i m}^{n+1}+\left(1-\theta_{D}\right) C_{i m}^{n}\right]
\end{aligned}
$$

$$
\frac{C_{i m}^{n+1}-C_{i m}^{n}}{h}=\beta_{i}\left[\theta_{p} F_{m}^{n+1} \phi_{m}^{n+1}+\left(1-\theta_{p}\right) F_{m}^{n} \phi_{m}^{n}\right]-\lambda_{i}\left[\theta_{D} C_{i m}^{n+1}+\left(1-\theta_{D}\right) C_{i m}^{n}\right]
$$

where $\theta_{p}$ and $\theta_{D}$ are "implicity constants" such that $0 \leqslant \theta_{p} \leqslant 1$ and $0 \leqslant \theta_{D} \leqslant 1$. This finite difference formulation, Eq. (2.18), is the form the TWIGL code ${ }^{11}$ equations would take on if TWIGL were reduced to one energy group and 
one spatial dimension. These equations will hereafter be referred to as the WIGL' equations.

Note that the nuclear parameters in Eq. (2.18) are evaluated at two levels in time. A two-level kinetics matrix discretization is used to conform with the WIGLE 10 and TWIGL 11 approach and also because of the anticipated advantage which was discussed in Section 2.If.

2.2b Application of the Prompt Jump Approximation to the Spatially Dependent Finite Difference Equations

The prompt jump approximation may be applied to the spatially dependent neutron diffusion equation by setting the neutron flux, $\phi(x, t)$, instantaneously into equilibrium with the delayed neutron precursor concentrations. The resulting prompt jump neutron diffusion equation is

$$
0=G(x, t) \phi(x, t)+(1-\beta) F(x, t) \phi(x, t)+\sum_{i=1}^{I} \lambda_{i} C_{i}(x, t)
$$

where the precursor concentrations are found by

$$
\frac{\partial C_{i}(x, t)}{\partial t}=\beta_{i} F(x, t) \phi(x, t)-\lambda_{i} C_{i}(x, t) \quad i=1,2, \cdots, I
$$

By this formulation, $\phi(x, t)$ is in a sense directly proportional to the $C_{i}(x, t)$ terms.

The finite difference theta method form of the spatially dependent prompt jump equations is 


$$
\begin{aligned}
0=G_{m}^{n+1} & \phi_{m}^{n+1}+(1-\beta) F_{m}^{n+1} \phi_{m}^{n+1} \\
& +\sum_{i=1}^{I} \lambda_{i} C_{i m}^{n+1}
\end{aligned}
$$

$$
\begin{array}{r}
\frac{C_{i m}^{n+1}-C_{i m}^{n}}{h}=\beta_{i}\left[\theta_{p} F_{m}^{n+1} \phi_{m}^{n+1}+\left(1-\theta_{p}\right) F_{m}^{n} \phi_{m}^{n}\right] \\
-\lambda_{i}\left[\theta_{D} C_{i m}^{n+1}+\left(1-\theta_{D}\right) C_{i m}^{n}\right] \\
i=1,2, \cdots, I
\end{array}
$$

where again $0 \leq \theta_{p} \leq 1$ and $0 \leq \theta_{D} \leq 1$ These equations will hereafter be referred to as the PJ equations.

Note that if $\theta_{p}=\theta_{D}=1$ in both the WIGL' and PJ equations, then the PJ equations are the same as the WIGL' equations with $\frac{1}{v}$ set equal to zero.

A computer code, WIGLPJ, has been written to solve both the WIGL' and PJ equations. In the next section numerical results generated by this code are used to compare the WIGL' and PJ methods. 


\subsection{Numerical Results}

The applicability of the prompt jump approximation to spatially dependent kinetics problems may be assessed by numerical testing. In this section the results of several numerical tests are presented.

Eash test problem presented in this section represents a perturbation from one of three critical configurations. The geometry and physical properties of these configurations are described in detail in Appendix D. Only one-dimensional, single energy group problems are considered. Since results are relatively insensitive to the number of delayed neutron precursor groups, only one precursor group is used in each test problem.

Table 2.1 lists typical results for homogeneous positive step perturbations. At time $t=0$ the fission cross section is increased $.2 \%$ above its initial critical value. (See Critical Configuration No. 1 in Appendix D.) This corresponds to a reactivity insertion of about $\rho=\frac{1}{3} \beta$. The exact solution of this problem is provided by a point kinetics calculation.*

The results of Table 2.1 are illustrated in Figure 2.3. Figure 2.3 shows that the PJ solution only approximates the initial behavior of the neutron flux. and is

* The point kinetics model is as accurate as the diffusion theory model for homogeneous reactor configurations. 
TABLE 2.1

Test Problem 2.I: Homogeneous Positive Step Perturbation ( $\rho \approx \frac{1}{3} \beta$ ) Applied to the Reactor of Critical Configuration No. 1

$\theta_{P}=\theta_{D}=1 / 2$

Time step size $=.01$ seconds

\begin{tabular}{|l|lll|}
\cline { 2 - 4 } \multicolumn{1}{c|}{$\begin{array}{l}\text { Time } \\
\text { (sec) }\end{array}$} & $\begin{array}{l}\text { Centerpoint fluxes } \\
\text { Kinetics }\end{array}$ & PJ & WIGL' \\
\hline .01 & 1.274 & 1.496 & 1.142 \\
.02 & 1.397 & 1.497 & 1.346 \\
.03 & 1.452 & 1.498 & 1.432 \\
.06 & 1.495 & 1.500 & 1.493 \\
.1 & 1.502 & 1.504 & 1.502 \\
.2 & 1.511 & 1.513 & 1.511 \\
.3 & 1.520 & 1.522 & 1.520 \\
& & & \\
\hline
\end{tabular}

FIGURE 2.3

Semi-Log Plot of Table 2.1 Data

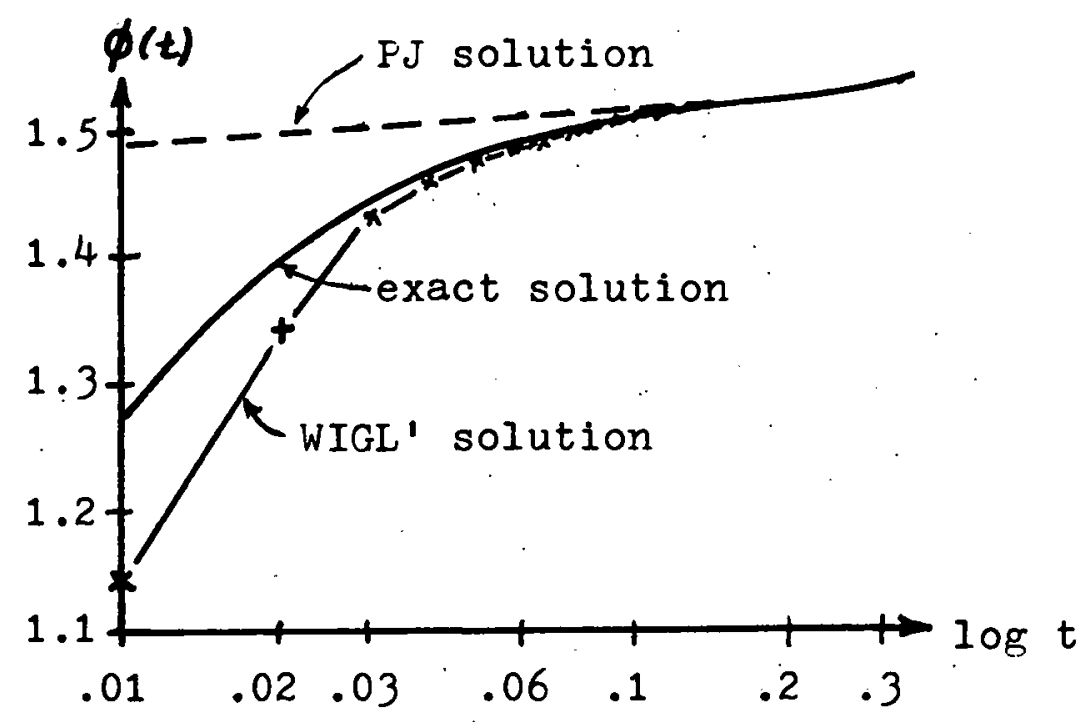


therefore in error during the initial part of the transient. The initial jump of the neutron flux is also poorly treated by the WIGL' solution because much smaller time step sizes are required in order to calculate the initial detailed behavior of the flux during the jump. Both the WIGL' and PJ solutions accurately model the asymptotic behavior of the neutron flux.

Table 2.2 lists typical results for ramp perturbations. The reactor of this problem is described by Critical configuration No. 2 in Appendix D. For the first . I second of the transient the fission cross section is linearly increased to .1\% above its initial critical value in one half of the reactor and decreased by $15 \%$ in the other half. During the first three seconds of the transient the flux tilts $15 \%$.

The WIGL' solution is converged and is therefore the true solution of this problem. Significantly, the PJ solution is also converged --.- but to a slightly different set of values. This is because the PJ solution converges to the solution of the prompt jump equations, Eqs. (2.19), and not to the solution of the neutron diffusion equations, Eqs. (2.16).

A nonlinear perturbation is applied to the reactor of Critical Configuration No. 1 in the problem of Table 2.3. In this problem the fission cross section varies sinusoidally with a period of $T=2 \pi$ seconds according to 
TABLE 2.2

Test' Problem 2.2: Nonuniform Ramp Perturbation Applied to the Reactor of Critical Configuration No: 2

$\theta_{p}=\theta_{D}=\frac{1}{2}$

Time Step Size $=.01$ seconds

\begin{tabular}{|c|c|c|}
\hline \multirow[b]{2}{*}{$\begin{array}{l}\text { Time } \\
\text { (sec) }\end{array}$} & \multicolumn{2}{|c|}{$\begin{array}{l}\text { Tabulated flux measured at } \\
\text { center of reactor between } \\
\text { two nonuniformly perturbed } \\
\text { regions }\end{array}$} \\
\hline & WIGL' & PJ \\
\hline $\begin{array}{l}.01 \\
.03 \\
.06\end{array}$ & $\begin{array}{r}9998 \\
.9981 \\
.9933\end{array}$ & $\begin{array}{r}.9965 \\
.9898 \\
.9806\end{array}$ \\
\hline $\begin{array}{r}.1 \\
.3 \\
1.0 \\
3.0\end{array}$ & $\begin{array}{l}.9844 \\
.9703 \\
.9680 \\
.9638\end{array}$ & $\begin{array}{l}.9697 \\
.9692 \\
.9677 \\
.9634\end{array}$ \\
\hline
\end{tabular}


TABLE 2.3

Test Problem 2.3: Sinusoidal

Perturbation of the Reactor of Critical Configuration No. 1

$\theta_{p}=\theta_{D}=\frac{1}{2}$

Time Step Size $=.01$ seconds

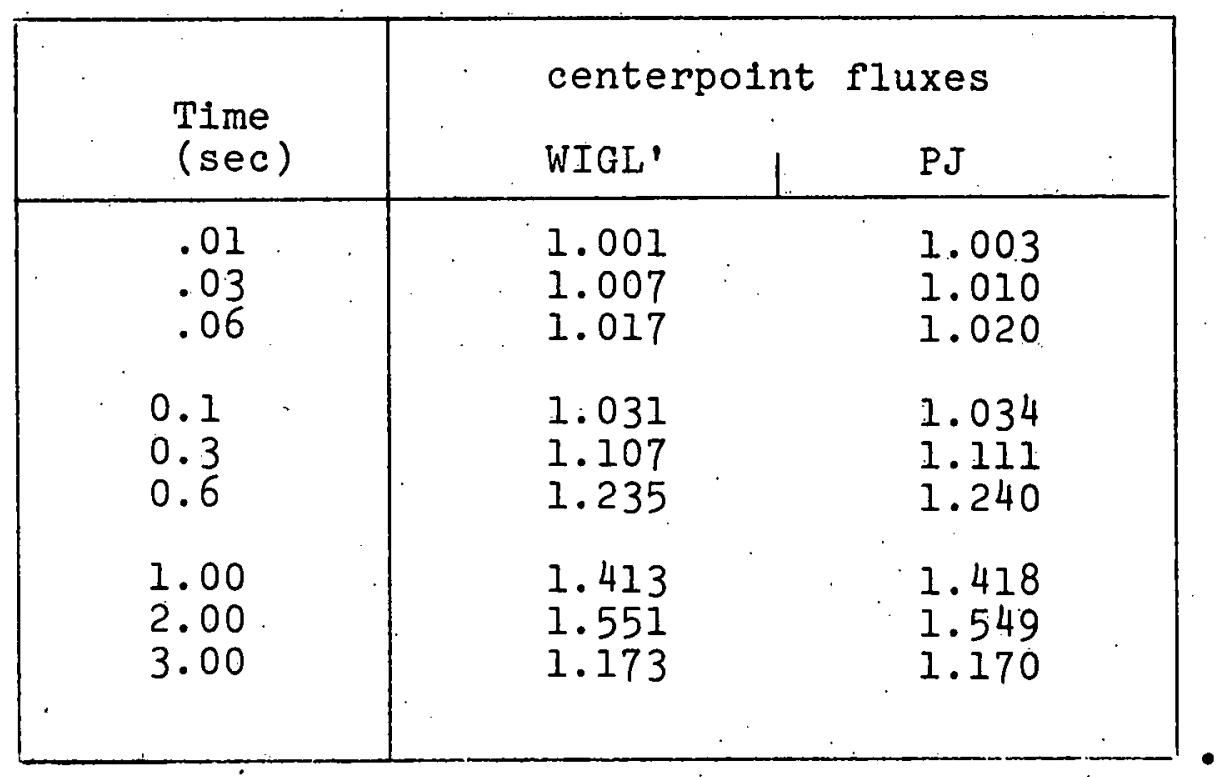




$$
\Sigma_{f}(t)=(1.0+0.002 \sin t) \cdot \Sigma_{f}(0)
$$

The PJ solution agrees well with the converged WIGL' solution because the transient period, $T$, is large compared with the delayed neutron precursor decay constant, $\lambda$, which has the value $\lambda=.1 \mathrm{sec}^{-1}$ for this problem. However, for oscillating perturbation problems where $T \approx \lambda$ or $T<\lambda$ the $P J$ solution may be very inaccurate and converge to an erroneous solution. This is because the neutron flux in the prompt jump approximation is set instantaneously in equilibrium with the delayed neutron precursors as in Eq. (2.20). When $T \gg \lambda$, the delayed neutron precursors follow the transient well and the PJ solution is accurate. But for oscillating perturbation problems with short periods, or for any rapidly fluctuating perturbation problems, it is no longer accurate to assume that the prompt neutrons are in equilibrium with the delayed neutrons. The prompt jump approximation is unsuitable for these problems.

Table 2.4 illustrates typical results for negative step perturbations. The reactor modeled in this problem is described by Critical Configuration No. 3 in Appendix D. The transient is initiated by increasing the capture 
TABLE 2.4

Test Problem 2.4: Nonuniform Negative Step Perturbation Applied to the Reactor of Critical Configuration No. 3

Tabulated fluxes are measured $1 / 4$ way across the reactor in the center of the perturbed half.

Time step size $=0.1$ seconds

2. $4 \mathrm{a} \quad \theta_{p}=\theta_{D}=\frac{1}{2}$

\begin{tabular}{|c|c|c|c|}
\hline $\begin{array}{l}\text { Time } \\
(\mathrm{sec})\end{array}$ & PJ & WIGL! & $\begin{array}{l}\text { single-level } \\
\text { formulation }\end{array}$ \\
\hline $\begin{array}{l}0.0 \\
.1 \\
.2 \\
.3 \\
.4 \\
.5\end{array}$ & $\begin{array}{r}.7071 \\
.4942 \\
.4927 \\
.4913 \\
.4898 \\
.4884\end{array}$ & $\begin{array}{l}.7071 \\
.5057 \\
.4827 \\
.5004 \\
.4819 \\
.4956\end{array}$ & $\begin{array}{l}.7071 \\
.3042 \\
.6611 \\
.3397 \\
.6241 \\
.3671\end{array}$ \\
\hline $\begin{array}{r}.6 \\
.7 \\
.8 \\
.9 \\
1.0\end{array}$ & $\begin{array}{l}.4870 \\
.4855 \\
.4841 \\
.4827 \\
.4813\end{array}$ & $\begin{array}{l}.4807 \\
.4913 \\
.4792 \\
.4872 \\
.4774\end{array}$ & $\begin{array}{r}.5944 \\
.3882 \\
.5703 \\
.4042 \\
.5506\end{array}$ \\
\hline
\end{tabular}

$2.4 \mathrm{~b} \quad \theta_{p}=\theta_{D}=1.0$

\begin{tabular}{|c|ll|}
\hline $\begin{array}{c}\text { Time } \\
(\mathrm{sec})\end{array}$ & PJ & WIGL \\
\hline 0.0 & .7071 & .7071 \\
.1 & .4934 & .4994 \\
.2 & .4920 & .4923 \\
.3 & .4906 & .4906 \\
.4 & .4891 & .4892 \\
.5 & .4877 & .4878 \\
.6 & .4863 & .4864 \\
.7 & .4849 & .4849 \\
.8 & .4834 & .4835 \\
.9 & .4820 & .4821 \\
1.0 & .4806 & .4807 \\
\hline
\end{tabular}


cross section by $1.36 \%$ in one half of the reactor. The resulting global reactivity is approximately $\rho \approx-\frac{1}{2} \beta$.

The PJ solution tabulated in Table $2.4 \mathrm{a}$ is accurate to within .2\%. (This small error is due mostly to the time lag effect mentioned in Section 2.1f.) The less accurate WIGL' solution oscillates about the PJ solution. For this problem the time step size used in the PJ equations may be 5. - 10 times larger than the time step size used in the WIGL' equations for a given accuracy.

The WIGL' solution in Table $2.4 \mathrm{a}$ is of acceptable accuracy because a two-level kinetics matrix (Section 2.If) is used in the WIGL' difference system. If a single level kinetics matrix is used, the resulting solution is unacceptable. This is shown by the last column of Table 2.4a in which a single-level. version of WIGL' is tested. The superiority of the PJ method for negative step perturbations follows from the point kinetics arguments of Section 2.1. Figure 2.2 shows that $\left|\omega_{I}\right|$ is largest for negative $\left(\rho_{k}<0\right)$ reactivities. Hence the argument of Section 2.1 indicates that the advantage of the prompt jump approximation is most pronounced for negative step perturbations.

In Table $2.4 \mathrm{~b}$ the implicity constants, $\theta_{\mathrm{p}}$ and $\theta_{\mathrm{D}}$, are set equal to unity in both the WIGL' and PJ formulations. (This choice of implicity constants is often best for negative reactivity insertion problems.) As a result, 
the PJ solution is now accurate to $.01 \%$ and the WIGL' solution is also very accurate. In general, an optimal choice of $\theta_{p}$ and $\theta_{D}$ lessens any advantage of using the prompt jump approximation.

\section{$2.4^{\circ}$ Conclusions}

The prompt jump approximation is applicable to spatially dependent reactor kinetics. However, there are some limitations to this method:

1.) Although the prompt jump approximation is applicable to subcritical and supercritical transient problems, it is invalid for prompt supercritical problems;

2) Only asymptotic flux behavior is modeled accurately when the prompt jump approximation is used. For problems in which there is no asymptotic solution, application of the prompt jump approximation leads to erroneous results;

3) The solution of the prompt jump approximated equations does not converge to the solution of the unapproximated equations but rather to the asymptotic solution of the unapproximated equations.

The above limitations only apply to two classes of problems: prompt supercritical problems and problems with a non-asymptotic solution. For other problems, the prompt jump approximation may be applied successfully. In section 2.3 it was shown that the prompt jump approximation is 
applicable to step perturbation, ramp perturbation, and nonlinear perturbation problems.

When the prompt jump approximation is applied to the neutron diffusion equation and a theta method solution technique is used, the approximate prompt jump solution is generally competitive with the unapproximated theta method solution in terms of accuracy for a given time step size. Whether or not there is any advantage in using the prompt jump approximation depends upon the problem considered and the theta method used. For some problems, particularly negative step perturbation problems, use of the prompt jump approximation may make larger time step sizes possible. 
Chapter. 3

THE ALTERNATING DIRECTION CHECKERBOARD METHOD

The alternating direction checkerboard method is a recently proposed 17 alternating direction method. This method and its variations will be examined in sections 3.2 - 3.5, but first it is useful to discuss some other alternating direction methods in detail.

\subsection{Review of Alternating Direction Methods}

As described in Chapter 1 , the finite difference multigroup neutron diffusion equation may be written in matrix form as

$$
\frac{\partial \bar{\psi}}{\partial t}=A \bar{\psi}
$$

This may be solved by either a one-step or a two-step finite difference approach. In either case, temporal discretization leads to

$$
\frac{\Psi^{n+1}-\bar{\psi}^{n}}{h}=\underline{A}_{1} \bar{\psi}^{n}+\underline{A}_{2} \bar{\psi}^{n+1}
$$

where

$$
\begin{aligned}
& \underline{A}=\underline{A}_{1}+\underline{A}_{2} \\
& \bar{\psi}^{n}=\text { flux vector at time } t^{n} \\
& \bar{\psi}^{n+1}=\text { flux vector at time } t^{n+1} \\
& h=t^{n+1}-t^{n}=\text { time step size }
\end{aligned}
$$

This may be rearranged as 


$$
\bar{\psi}^{n+1}=\left[I-h \underline{A}_{2}\right]^{-1}\left[\underline{I}+h \underline{A}_{1}\right] \bar{\psi}^{n}
$$

Eq. (1.9c) is the single-step finite difference solution of $\mathrm{Eq} \cdot(1.4)$.

In a two-step finite difference method, a second difference equation is introduced:

$$
\frac{\Psi^{n+2}-\bar{\psi}^{n+1}}{h}=A_{3} \bar{\psi}^{n+1}+A_{4} \bar{\psi}^{n+2}
$$

where

$$
\begin{aligned}
\underline{A} & =\underline{A}_{3}+\underline{A}_{4} \\
\bar{\psi}^{n+2} & =\text { flux vector at time } t^{n+2} \\
h & =t^{n+2}-t^{n+1}=t^{n+1}-t^{n}
\end{aligned}
$$

Eq. (1.9b) may be rearranged as

$$
\bar{\psi}^{n+2}=\left[I-h \underline{A}_{4}\right]^{-1}\left[\underline{I}+h \underline{A}_{3}\right] \bar{\psi}^{n}
$$

Eqs. (1.9a) and (1.9b) are the two equations of the two-step approach. These two steps, or "half-steps" as they are more commonly called, may be treated together by inserting Eq. (1.9c) into Eq. (1.9d) to yield

$$
\bar{\psi}^{n+2}=\left[\underline{I}-h \underline{A}_{4}\right]^{-1}\left[\underline{I}+h \underline{A}_{3}\right]\left[\underline{I}-h \underline{A}_{2}\right]^{-1}\left[I+h A_{1}\right] \Psi^{n}
$$

Eq. (1.10) is the two-step finite difference solution of Eq. (1.4). 
In order to assure that Eq. $(1.9 \mathrm{c})$ is numerically stable, it is usually necessary to choose $\underline{A}_{1}$ and $\underline{A}_{2}$ such that $\left[\mathrm{I}-\mathrm{h} \mathrm{A}_{2}\right]$ must be inverted iteratively in Eq. (1.9c). Therefore, single-step methods, such as theta methods, usually require a cumbersome iterative matrix inversion. For two step methods, $\underline{A}_{2}$ and $\underline{A}_{4}$ may be chosen so that the matrices $\left[I-h \underline{A}_{2}\right]$ and $\left[I-h \underline{A}_{4}\right]$ are inverted easily in Eq. (1.10). Any resulting instability of either Eq. (1.9c) or Eq. (1.9d) is inconsequential if Eq. (1.10) is numerically stable.

Alternating direction methods are two-step finite difference methods for which $\underline{A}_{1}, \underline{A}_{2}, \underline{A}_{3}$ and $\underline{A}_{4}$ are chosen

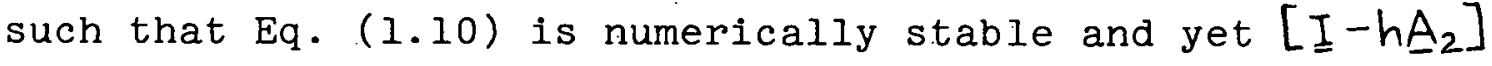
and $\left[\underline{I}-h \underline{A}_{4}\right]$ are easịly inverted. These methods may be divided into three categories differentiated by the treatment of diffusion terms: implicit, explicit, and checkerboard. The latter classification is new to reactor physics and will be described in detail later. The first two categories are traditional and provide an introduction to alternating direction methods; the best of these methods is the standard against which the checkerboard method is compared.

\section{1a Implicit Methods}

Alternating direction implicit (ADI) methods ${ }^{18}$ treat diffusion terms implicitly in one spatial dimension and explicitly in all others for each half-step of the two-step 
alternating direction approach.: In order to illustrate this, consider the first half-step for a two-dimensional problem in rectangular $x-y$ geometry. To solve for the flux at time $t^{n+1}$ in terms of the flux at time $t^{n}$ the diffusion term at each mesh point is treated by central differences as

$$
\begin{aligned}
&\left.D_{g} \nabla^{2} \phi_{g}\left(\bar{r}_{k, j}, t\right)\right|_{t^{n}} ^{t^{n+1}} \\
&=D_{g}\left[\frac{\phi_{9, k+1, j}^{n+1}-2 \phi_{g, k, j}^{n+1}+\phi_{g, k-1, j}^{n+1}}{h_{x}^{2}}+\frac{\phi_{g, k, j+1}^{n}-2 \phi_{g, k, j}^{n}+\phi_{g, k, j-1}^{n}}{h_{y}^{2}}\right] \\
& \text { where } \\
& k=\begin{aligned}
j= & \text { y-direction mesh index } \\
g= & \text { energy group index } \\
= & \text { spatial vector associated with mesh } \\
& \text { point (k,j) } \\
\bar{r}_{k, j}= & \text { diffusion coefficient for energy group g. } \\
& \text { (This is assumed to be constant in space } \\
& \text { and time for simplicity.) }
\end{aligned} \\
&
\end{aligned}
$$




$$
\begin{aligned}
\phi_{g, k, j}^{n}= & \text { group } g \text { neutron flux at mesh }(k, j) \text { at } \\
& \text { time } t^{n} \\
h_{x}= & x \text {-direction mesh spacing } \\
h_{y}= & y \text {-direction mesh spacing }
\end{aligned}
$$

It is seen from Eq. (3.1) that diffusion in the $x$-direction is treated implicitly. (that is, at time level $t^{n+l}$ ) and diffusion in the $\mathrm{y}$-direction is treated explicitly (at time level $t^{\mathrm{n}}$ ).

The order of solution may be described by considering the reactor mesh grid of Figure 3.1. First the fluxes at the $\mathrm{K}$ mesh points of grid row $j=1$ are solved simultaneously. This simultaneous solution requires a tridiagonal matrix inversion as will be shown shortly. Then the fluxes of succeeding rows, $j=2,3, \ldots, J$, are simultaneously. determined by tridiagonal inversions. As each grid row is treated, both old and new values of the fluxes must be stored so that the old values are available for the explicit part of the solution of the next grid row.

For the second half-step the direction of solution is alternated, hence the name alternating direction method. Now the fluxes of the $J$ mesh points in each mesh grid column are determined simultaneously for columns $k=1,2, \ldots, k$. 


\section{FIGURE 3.1}

Reactor Mesh Grid

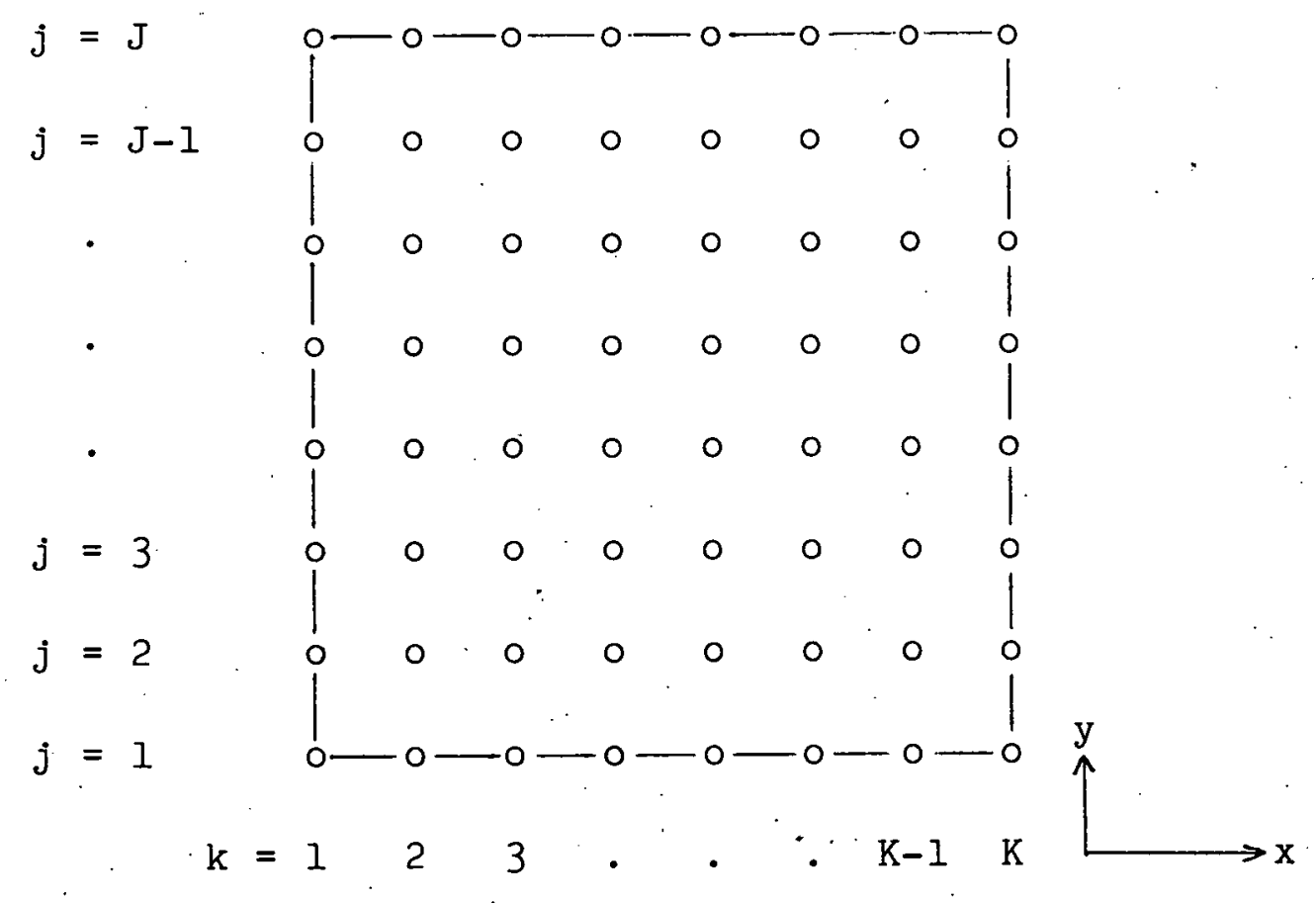


Again auxiliary storage is required and $\mathrm{K}$ tridiagonal. JxJ matrices must be inverted. The treatment of diffusion terms for this second half-step is.

$$
\begin{aligned}
& \left.D_{g} \nabla^{2} \phi_{g}\left(\bar{r}_{k, j}, t\right)\right|_{t^{n+1}} ^{t^{n+2}} \\
= & D_{g}\left[\frac{\phi_{9, k+1, j}^{n+1}-2 \phi_{g, k, j}^{n+1}+\phi_{g, k-1, j}^{n+1}}{h_{x}^{2}}+\frac{\phi_{g, k, j+1}^{n+2}-2 \phi_{g, k, j}^{n+2}+\phi_{g, k, j-1}^{n+2}}{h_{y}^{2}}\right]
\end{aligned}
$$

where diffusion in the $\mathrm{x}$-direction is now treated explicitly and diffusion in the y-direction is treated implicitly.

In matrix form, let the A-matrix of Eq. (1.4a),

$$
\frac{\partial \bar{\psi}}{\partial t}=\underline{A}
$$

be divided as

$$
\underline{A}=\underline{D}+\underline{R}
$$

where 


$$
\underline{D}=\underline{D}_{x}+\underline{D}_{y}
$$

and

$$
\underline{R}=\underline{R}_{1}+\underline{R}_{2}=\underline{R}_{3}+\underline{R}_{4}
$$

Let $\underline{D}$ be composed of all diffusion terms and the diagonal terms of the precursor equations. (It should be recalled that the delayed neutron precursor equations are incorporated into the A-matrix.) Let $\underline{D}_{x}$ include all terms associated with diffusion in the $x$-direction and let $\underline{D}_{y}$ include all terms associated with diffusion in the y-direction. Schematically, the matrices $\underline{D}, \underline{D}_{x}$, and $\underline{D}_{y}$ are
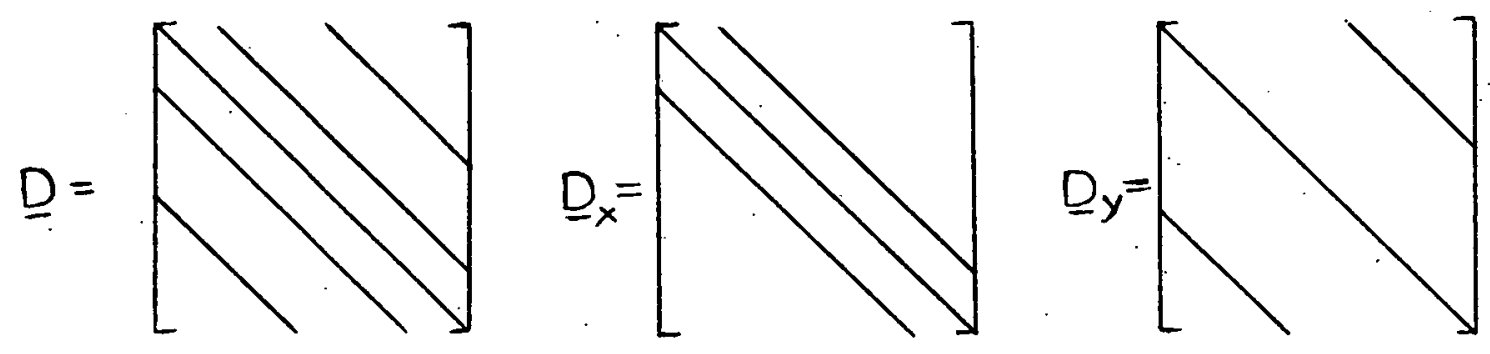

That is, in two dimensions, $\underline{D}$ is a five stripe matrix (see Section 1.4 and Appendix A.4); the matrices $\underline{D}_{x}$ and $\underline{D}_{y}$ are tridiagonal matrices. The tridiagonal form of $\underline{D}_{x}$ and 
$\underline{D}_{y}$ is why tridiagonal matrix inversions are required as each line of fluxes is solved simultaneously.

The ADI matrix splitting is given by

$$
\begin{aligned}
& \underline{A}_{1}=\underline{D}_{y}+\underline{R}_{1} \\
& \underline{A}_{2}=\underline{D}_{x}+\underline{R}_{2} \\
& \underline{A}_{3}=\underline{D}_{x}+\underline{R}_{3} \\
& \underline{A}_{4}=\underline{D}_{y}+\underline{R}_{4}
\end{aligned}
$$

This splitting corresponds to the two-dimensional treatment of diffusion terms in Eqs. (3.1) and (3.2). For three dimensions either a much more difficult three-step approach is required or a two-step approach for which $\underline{D}_{\mathbf{X}}$ and $\underline{D}_{y}$ are pentadiagonal is required. Either way, the ADI method is not easily extended beyond two dimensions.

\section{3.ib Explicit Methods}

Like ADI methods, alternating direction explicit (ADE) methods treat diffusion terms half explicitly and half implicitly. However, tridiagonal matrix inversions and auxiliary storage requirements are avoided by sweeping the spatial mesh point by point.

In order to illustrate the ADE method, again consider the reactor mesh grid of Figure 3.1. For the first ADE half-step fluxes are solved point by point along each row of the mesh grid moving from left to right, starting from grid row $j=1$ and finishing with grid row $j=\mathrm{J}$. 
For the second half-step the direction of solution is alternated; fluxes are solved point by point moving from right to left along each grid row starting from grid row. $j=J$ and finishing with row $j=1$.

Whereas alternating direction methods are classified according to the treatment of diffusion terms, it is useful to examine the $A D E$ treatment of diffusion during each half-step. In order to solve for the flux at time $t^{n+1}$ in terms of the flux at time $t^{n}$ the diffusion term at each mesh point is treated by central differences in the first half-step as

$$
\begin{aligned}
& \left.D_{g} \nabla^{2} \phi_{g}\left(\bar{r}_{k, j}, t\right)\right|_{t^{n}} ^{t^{n+1}} \\
& =D_{g}\left[\frac{\phi_{g_{k+1, j}}^{n}-\phi_{g k, j}^{n}-\phi_{g, k, j}^{n+1}+\phi_{g, k-1, j}^{n+1}}{h_{x}^{2}}+\frac{\phi_{g, k, j+1}^{n} \phi_{g_{, k, j}}^{n}-\phi_{g, k, j}^{n+1}+\phi_{g, k, j-1}^{n+1}}{h_{y}^{2}}\right] .
\end{aligned}
$$

Again the diffusion coefficient has been assumed to be constant for simplicity. The fluxes $\phi_{g_{k+1, j}}^{n}, \phi_{g_{, k, j}}^{n}$ and $\phi_{g, k, j+1}^{n}$ of the lower time level, $t^{n}$, are known from the previous time step calculation. The fluxes $\phi_{g k-1, j}^{n+1}$ and $\phi_{g_{k, j-1}^{n+1}}$ of the upper time level, $t^{n+1}$, are also known because the spatial mesh solution has already swept past 
these mesh grid points. Therefore only $\phi_{g, k, j}^{n+1}$ is unknown, and no tridiagonal matrix inversion is necessary. It is as if all fluxes were calculated explicitly. The downward and leftward sweep of the second half-step is explicit in the same sense.

In matrix form let the A-matrix be divided as in Eq. $(3 \cdot 3 a)$ :

$$
\underline{A}=\underline{D}+\underline{R}
$$

Again $D$ is composed of all diffusion terms and the diagonal terms of the precursor equation; the remaining elements of A are in $\underline{R}$. Again, $\underline{D}$ is a five stripe pentadiagonal matrix for two-dimensional problems. For ADE methods $\underline{D}$ is divided into $\underline{D}_{1}$ and $\underline{D}_{2}$ where $\underline{D}_{1}$ contains the two upper diagonal stripes and half the diagonal elements of $\underline{D} ; \underline{D}_{2}$ contains the two lower diagonal stripes and half the diagonal elements of $\underline{D}$. . With $\underline{R}$ divided as

$$
\underline{R}=\underline{R}_{1}+\underline{R}_{2}=\underline{R}_{3}+\underline{R}_{4}
$$

the ADE matrix splitting is then

$$
\begin{aligned}
& \underline{A}_{1}=\underline{D}_{1}+\underline{R}_{1} \\
& \underline{A}_{2}=\underline{D}_{2}+\underline{R}_{2} \\
& \underline{A}_{3}=\underline{D}_{2}+\underline{R}_{3} \\
& \underline{A}_{4}=\underline{D}_{1}+\underline{R}_{4}
\end{aligned}
$$


This two step approach is applicable to any number of dimensions, the only difference being the number of stripes in $\underline{D}_{1}$ and $\underline{D}_{2}$. For $N$ dimensions, $\underline{D}_{1}$ and $\underline{D}_{2}$ have $N+1$ stripes.

If $\underline{R}_{1}, \underline{R}_{2}, \underline{R}_{3}$ and $\underline{R}_{4}$ are chosen to be upper or lower triangular then the matrices to be inverted in the $\mathrm{ADE}$ method are always upper or lower triangular, or can be made so by rearranging the order of unknowns. The two half-steps may then be calculated simply by forward substitution ${ }^{9}$.

\section{1c The Exponential Transformation}

A change of variables has been found 19, 20 to improve dramatically implicit and explicit alternating direction methods. This change of variables, known as the exponential transformation, is

$$
\bar{\psi}=e^{\Omega t} \bar{\phi}
$$

where $\Omega$ is a diagonal matrix of spatially dependent frequencies. which are computed at each time step;

$\bar{\psi}$ is the multigroup flux vector;

$\bar{\phi}$ is the transformed flux vector.

Because the solution of the neutron diffusion equation, $\bar{\psi}$, is basically exponential in nature, the function $\bar{\phi}$ should be slowly varying in time provided $\Omega$ is properly chosen. If $\bar{\phi}$ rather than $\bar{\psi}$ is solved for, larger time step sizes may be possible. 
83

In order to obtain an equation for $\bar{\phi}$, the transformed flux vector is differentiated:

$$
\begin{aligned}
\frac{\partial \bar{\phi}}{\partial t} & =\frac{\partial}{\partial t}\left[e^{-\Omega t} \bar{\psi}\right] \\
& =e^{-\Omega t} \frac{\partial \bar{\psi}}{\partial t}-\Omega e^{-\Omega t} \bar{\psi} \\
& =e^{-\Omega t} \underline{\Delta} \bar{\psi}-\underline{\Omega} e^{-\Omega t} \bar{\psi}
\end{aligned}
$$

The transformed neutron diffusion equation is then

$$
\frac{\partial \bar{\phi}}{\partial t}=e^{-\underline{\Omega} t}(\underline{A}-\underline{\Omega}) e^{\underline{\Omega} t} \bar{\phi}
$$

The finite difference alternating direction method formulatron of this equation is

$$
\begin{aligned}
& \frac{\bar{\phi}^{n+1}-\bar{\phi}^{n}}{h}=e^{-\Omega t}\left(\underline{A}_{1}-\gamma \Omega\right) e^{\Omega t} \bar{\phi}^{n}+e^{-\Omega t}\left(\underline{A}_{2}-\alpha \Omega\right) e^{\Omega t} \bar{\phi}^{n+1} \\
& \frac{\bar{\phi}^{n+2}-\bar{\phi}^{n+1}}{h}=e^{-\Omega t}\left(\underline{A}_{3}-\gamma \Omega\right) e^{\Omega t} \phi^{n+1}+e^{-\Omega t}\left(\underline{A}_{4}-\alpha \Omega\right) e^{\Omega t} \bar{\phi}^{n+2}
\end{aligned}
$$

$(3.5 b)$ 
84

where $\alpha$ and $\gamma$ are any two numbers such that $\alpha+\gamma=1$.

These equations may be combined to eliminate $\bar{\phi}^{n+1}$ :

$$
\begin{aligned}
& \bar{\phi}^{n+2}=\left[\underline{I}-h e^{-\underline{\Omega} t}\left(\underline{A}_{4}-\alpha \underline{\Omega}\right) e^{\Omega} \underline{\underline{\Omega}}\right]^{-1}\left[\underline{\underline{I}}+h e^{-\underline{\Omega} t}\left(\underline{A}_{3}-\gamma \underline{\Omega}\right) e^{\underline{\Omega} t}\right] \\
& \times\left[I-h e^{-\Omega t}\left(A_{2}-\alpha \Omega\right) e^{\Omega t}\right]^{-1}\left[\underline{I}+h e^{-\Omega t}\left(A_{i} \gamma \Omega\right) e^{\Omega} t\right] \Phi^{n} \\
& =e^{-\Omega t}\left[\underline{I}-h\left(\underline{A}_{4}-\alpha \underline{\Omega}\right)\right]^{-1}\left[I+h\left(\underline{A}_{3}-\gamma \underline{\Omega}\right)\right] \\
& \times\left[\underline{I}-h\left(\underline{A}_{2}-\alpha \Omega\right)\right]^{-1}\left[\underline{I}+h\left(\underline{A}_{1}-\gamma \Omega\right)\right] e^{\Omega} \underline{\underline{ }} \bar{\phi}^{n}
\end{aligned}
$$

Evaluating the exponentials at $t=t^{n+1}$, midway through the time interval, then yields a finite difference alterrating direction method equation for $\bar{\phi}^{n+2}$ :

$$
\begin{aligned}
\bar{\phi}^{n+2}= & e^{-\Omega t^{n+1}}\left[\underline{I}-h\left(\underline{A}_{4}-\alpha \Omega\right)\right]^{-1}\left[\underline{I}+h\left(\underline{A}_{3}-\gamma \Omega\right)\right] \\
& \times\left[\underline{I}-h\left(\underline{A}_{2}-\alpha \Omega\right)\right]^{-1}\left[\underline{I}+h\left(\underline{A}_{1}-\gamma \Omega\right)\right] e^{\Omega} t^{n+1} \bar{\phi}^{n}
\end{aligned}
$$

For the multigroup flux vector, $\bar{\psi}$, to be found by the exponential transformation method, use is made of Eq. $(3.4):$

$$
\bar{\psi}^{n+2}=e^{\Omega t^{n+2}} \bar{\phi}^{n+2} ; \quad ; \quad \bar{\phi}^{n}=e^{-\Omega t^{n}} \cdot \bar{\psi}^{n}
$$


Using Eqs. (3.7) in Eq. (3.6) leads to

$$
\begin{aligned}
\bar{\psi}^{n+2}= & e^{\Omega t^{n+2}} e^{-\Omega t^{n+1}}\left[I-h\left(A_{4}-\alpha \Omega\right)\right]^{-1}\left[I+h\left(A_{3}-\gamma \Omega\right)\right] \\
& \times\left[I-h\left(\underline{A}_{2}-\alpha \Omega\right)\right]^{-1}\left[I+h\left(A_{1}-\gamma \Omega\right)\right] e^{\Omega t^{n+1}} e^{-\Omega t^{n}} \bar{\psi}^{n}
\end{aligned}
$$

or

$$
\begin{aligned}
\bar{\psi}^{n+2}= & e^{\Omega h}\left[I-h\left(\underline{A}_{4}-\alpha \Omega\right)\right]^{-1}\left[\underline{I}+h\left(\underline{A}_{3}-\gamma \underline{\Omega}\right)\right] \\
& \times\left[I-h\left(A_{2}-\alpha \Omega\right)\right]^{-1}\left[I+h\left(\underline{A}_{1}-\gamma \Omega\right)\right] e^{\Omega h} \bar{\psi}^{n}
\end{aligned}
$$

This is the governing equation of the alternating direction method with the exponential transform.

The way in which frequencies are chosen is important. Reed 19 has found that choosing each element of $\Omega$ as

$$
\Omega_{k j}^{n+2}=\frac{1}{2 h} \ln \frac{\psi_{G_{k, j}}^{n}}{\psi_{G k, j}^{n-2}}
$$

gives good results. As before, $n, k, j$, and $G$ refer to the time level, $\mathrm{x}$-mesh point, $\mathrm{y}$-mesh point and thermal neutron group. There seems to be no advantage to making the frequency energy group dependent, so only thermal group fluxes are used. At each spatial mesh point, both the frequency, $\Omega_{k, j}^{n}$, and the past thermal flux, $\psi_{G, k, j}^{n-2}$, require auxiliary storage. 
When the exponential transformation, or frequency transform method as it is also called, is applied as in the MITKIN 19 code, time step sizes may be increased as much as tenfold over those of the untransformed method. Therefore, the additional complexity of the equations and the auxiliary storage requirements are a worthwhile investment in computational effort.

\section{1d Truncation Errors}

The truncation error of any approximate method is found by comparing the approximate solution to the true solution of the governing equation. The true solution of the finite difference neutron diffusion equation,

$$
\frac{\partial \bar{\psi}}{\partial t}=A \bar{\psi}
$$

over the time interval

$$
t^{n}<t \leq t^{n+2}
$$

where

$$
2 h=t^{n+2}-t^{n}
$$

is

$$
\bar{\psi}_{\text {true }}^{n+2}=\exp (2 h \underline{-}) \bar{\psi}^{n}
$$


87

The exponential may be expanded by Taylor series yielding

$$
\Psi^{n+2}=\left[\underline{I}+2 h \underline{A}+2 h^{2} \underline{A}^{2}+\frac{4}{3} h^{3} \underline{A}^{3}+O\left(h^{4}\right)\right] \bar{\psi}^{n}
$$

To determine the truncation error associated with alternating direction methods, the true solution, Eq. (3.10), is compared to the alternating direction method solution,

$$
\begin{aligned}
\bar{\psi}^{n+2}= & e^{\Omega h}\left[\underline{I}-h\left(\underline{A}_{4}-\alpha \Omega\right)\right]^{-1}\left[I+h\left(\Delta_{3}-\gamma \Omega\right)\right] \\
& \times\left[\underline{I}-h\left(A_{2}-\alpha \Omega\right)\right]^{-1}\left[\underline{I}+h\left(A_{1}-\gamma \Omega\right)\right] e^{-} h \bar{\psi}^{n}
\end{aligned}
$$

Using the expansion

$$
[\underline{I}-h \underline{C}]^{-1}=\left[\underline{I}+h \underline{C}+h^{2} \underline{C}^{2}+h^{3} \underline{\underline{C}}^{3}+\cdots\right]
$$

(which is valid only if $\mathrm{h} \underline{\mathrm{C}}$ has a spectral radius less than unity) and expanding the $e^{\Omega h}$ terms as

$$
e^{\Omega h}=\underline{I}+h \underline{\Omega}+\frac{1}{2} h^{2} \underline{\Omega}^{2}+\frac{1}{6} h^{3} \Omega^{3}+\cdots
$$

converts alternating direction method solution, Eq. (3.8), to

$$
\begin{aligned}
\bar{\psi}^{n+2}= & \left\{\underline{I}+2 h \underline{A}+h^{2}\left[\left(\underline{A}+\underline{A}_{2}+\underline{A}_{4}\right) \underline{A}\right.\right. \\
& \left.\left.+\left(\underline{A}-\underline{A}_{2}-A_{4}\right) \Omega+(1-2 \alpha) \Omega(\underline{A}-\underline{\Omega})\right]+O\left(h^{3}\right)\right\} \bar{\psi}^{n}
\end{aligned}
$$

$(3.11)$. 
This is seen to agree with the true solution, Eq. (3.10), through terms of order $h$; that is, the truncation error is of order $h^{2}$ :

$$
\begin{aligned}
\text { T.E. } & =\bar{\psi}_{\text {true }}^{n+2}-\bar{\psi}^{n+2} \\
& =\left\{h^{2}\left[\left\{\underline{A}-\underline{A}_{2}-\underline{A}_{4}-(1-2 \alpha) \underline{\Omega}\right\}(\underline{A}-\underline{\Omega})\right]+O\left(h^{3}\right)\right\} \bar{\psi}^{n}
\end{aligned}
$$

An $\mathrm{h}^{3}$ order truncation error method can be achieved by choosing

$$
\begin{aligned}
& \underline{A}_{2}+\underline{A}_{4}=\underline{A} \\
& \alpha=\gamma=1 / 2
\end{aligned}
$$

It then follows that

$$
\underline{A}_{1}=\underline{A}_{4} ; \quad \underline{A}_{2}=\underline{A}_{3}
$$

and Eq. (3.8) becomes

$$
\begin{aligned}
\bar{\psi}^{n+2} & =\left\{\underline{I}+2 h \underline{A}+2 h^{2} \underline{A}^{2}+h^{3}\left[2\left(\underline{A}^{2}-\underline{A}_{2} \underline{A}_{1}\right) \underline{A}+2 \underline{A}_{2} \underline{A}_{1} \Omega\right.\right. \\
& +\Omega_{A_{1}} \underline{A}-\underline{A} \Omega-\underline{A}_{1} \Omega \underline{A}+\underline{A}_{1} \Omega^{2}-\Omega \underline{A}_{1} \Omega \\
& \left.\left.+\frac{1}{2} \Omega^{2} \underline{A}-\frac{1}{6} \Omega^{3}\right]+O\left(h^{4}\right)\right\} \bar{\psi}^{n}
\end{aligned}
$$

which agrees with Eq. (3.10) through terms of order $h^{2}$. A splitting for which $\underline{A}_{1}=\underline{A}_{4}$ and $\underline{A}_{2}=\underline{A}_{3}$ is known as a 
symmetric splitting. Splittings for which $\underline{A}_{2}+\underline{A}_{4} \neq \underline{A}$, as in Eq. (3.11), are known as nonsymmetric. The truncation error associated with symmetric splittings is of order $h^{3}$ :

$$
\begin{aligned}
\text { T.E. }= & \Psi_{\text {true }}^{n+2}-\bar{\psi}^{n+2} \\
= & \left\{h ^ { 3 } \left[2\left(\underline{A}_{2} \underline{A}_{1}-\frac{1}{3} \underline{A}^{2}\right) \underline{A}-2 \underline{A}_{2} \underline{A}_{1} \Omega-\Omega \underline{A}_{1} \underline{A}+\underline{\underline{A}} \underline{\underline{A}}\right.\right. \\
& \left.\left.+\underline{A}_{1} \underline{\Omega} \underline{A}-\underline{A}_{1} \underline{\Omega}^{2}+\underline{\Omega} \underline{A}_{1} \Omega-\frac{1}{2} \Omega^{2} \underline{A}+\frac{1}{6} \underline{\Omega}^{3}\right]+O\left(h^{4}\right)\right\} \bar{\psi}^{n}
\end{aligned}
$$

3.1e Comparison of Some Symmetric and Nonsymmetric Methods Four two-dimensional alternating direction schemes which use the frequency transform have been examined by Ferguson 21 to determine which is best. Each of these divides the A-matrix as

$$
\underline{A}=\underline{D}+\underline{U}+\underline{L}+\underline{T}
$$

Here $D$ contains all diffusion terms and the diagonal of the precursor equations as before; $\underline{U}, \underline{L}$, and $\underline{T}$ account for all remaining terms where $\underline{U}$ is strictly upper triangular. $\underline{L}$ is strictly lower triangular, and $\underline{T}$ is diagonal. Comparison with Eq. (3.3a) indicates that

$$
\underline{U}+\underline{L}+\underline{T}=\underline{R}
$$

The matrix $\underline{D}$ may be divided into $\underline{D}_{1}$ and $\underline{D}_{2}$ for explicit 
schemes or into $\underline{D}_{\mathrm{x}}$ and $\underline{D}_{\mathrm{y}}$ for implicit schemes as described earlier. The four methods examined by Ferguson are:

SADI (symmetric ADI) 20, 22, 23

$$
\begin{aligned}
& \alpha=\gamma=\frac{1}{2} \\
& \underline{A}_{1}=\underline{A}_{4}=\underline{D}_{y}+1 / 2 \underline{T}+\underline{U} \\
& \underline{A}_{2}=\underline{A}_{3}=\underline{D}_{x}+1 / 2 \underline{T}+\underline{L}
\end{aligned}
$$

NSADI (nonsymmetric ADI) 20, 23

$$
\begin{aligned}
\alpha & =0 ; \quad \gamma=0 \\
\underline{A}_{1} & =\underline{D}_{y}+U ; \quad \underline{A}_{3}=\underline{D}_{x}+\underline{U} \\
\underline{A}_{2} & =\underline{D}_{x}+\underline{T}+\underline{L} ; \quad \underline{A}_{4}=\underline{D}_{y}+\underline{T}+\underline{L}
\end{aligned}
$$

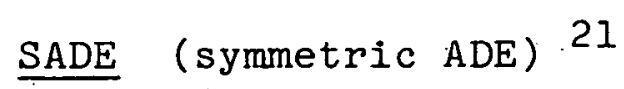

$$
\begin{aligned}
\alpha & =\gamma=1 / 2 \\
\underline{A}_{1} & =\underline{A}_{4}=\underline{D}_{1}+1 / 2 \underline{T}+\underline{U} \\
\underline{A}_{2} & =\underline{A}_{3}=\underline{D}_{2}+1 / 2 \underline{T}+\underline{L}
\end{aligned}
$$

NSADE (nonsymmetric ADE) 19, 21

$$
\begin{aligned}
& \alpha=I ; \quad \gamma=0 \\
& \underline{A}_{1}=\underline{D}_{1}+\underline{U} ; \quad \underline{A}_{3}=\underline{D}_{2}+\underline{U} \\
& A_{2}=\underline{D}_{2}+\underline{T}+\underline{L} ; \quad \underline{A}_{4}=\underline{D}_{1}+\underline{T}+\underline{L}
\end{aligned}
$$

Whereas the nondiffusion terms are treated in each of these methods: by either upper or lower triangular matrices, all four methods offer relative computational ease. They 
are also unconditionally stable ${ }^{19}$.

The multigroup symmetric methods are found to be less accurate than the nonsymmetric methods despite their higher order $h^{3}$ truncation error properties. This is thought to be because $20,21,23$ the SADE and SADI schemes sweep the energy mesh from the lowest energy group to the highest energy group in the second step of the two step approach. For thermal reactors the multigroup model is characterized by group structures with closely coupled down-scattering from each group to the next lower group. The groups are only coupled loosely by the upward flow of neutrons from fission because the fission spectrum is nonzero only for high energy groups. Inaccuracies result when the energy mesh is swept in the direction of loose coupling from the lowest energy group to the highest energy group as is done in the SADI and SADE methods.

In terms of truncation error analysis, the superiority of the nonsymmetric schemes is explained if it is assumed that the $h^{3}$ truncation error term of the symmetric schemes, Eq. (3.13), is larger than the $\mathrm{h}^{2}$ truncation error term, Eq. (3.12), of the nonsymmetric schemes.

It may be expected that a symmetric scheme in which the energy mesh is swept for both half-steps from highest to lowest energy group would be, superior to the nonsymmetric methods. Hansen 24 has tried such a scheme: 


$$
\begin{aligned}
& \underline{A}_{1}=\underline{A}_{4}=\underline{D}_{1}+1 / 2 \underline{R} \\
& \underline{A}_{2}=\underline{A}_{3}=\underline{D}_{2}+1 / 2 \underline{R}
\end{aligned}
$$

This method, hereafter referred to as the SIADE method, sweeps the energy mesh in the desired fashion. However, a time consuming $G \times G$ ( $G=$ the number of energy groups) matrix inversion is required at each spatial mesh point for both half steps because $\underline{A}_{1}, \underline{A}_{2}, \underline{A}_{3}$ and $\underline{A}_{4}$ are neither upper nor lower triangular. Evidently the $\mathrm{h}^{3}$ truncation error of this scheme is still at least as large as the $h^{2}$ error of the NSADE and NSADI methods because the SIADE method accuracy is comparable to that of the NSADE method. Therefore, the faster nonsymmetric schemes are preferable. Solutions obtained with the NSADE method are very similar to those obtained with the NSADI method. In the explicit method errors are observed to build up as the reactor mesh is swept from one corner to the diagonally opposite corner so that the flux exhibits a measurable tilt at the end of the first half-step ${ }^{21}$. These errors are cancelled in the second half-step as the mesh is swept in the opposite direction. Although the implicit methods do not exhibit these nonsymmetric tilts, the numerical solution is no better after the completion of both alternating direction steps ${ }^{21}$. 
Ferguson 2l. concludes that the NSADE method is the best of the above alternating direction schemes for four reasons:

1) the nonsymmetric splittings perform more satisfactorily in thermal reactor problems because the energy mesh is swept from fast groups to thermal groups;

2) the implicit methods are no more accurate than the explicit methods;

3) the explicit methods are computationally slightly faster because the tridiagonal matrix inversions and auxiliary storage of implicit methods are avoided;

4) the explicit methods are more readily extended to three dimensions.

Therefore, the NSADE method with the frequency transform (as programmed in the MITKIN ${ }^{19}$ code) has been chosen as the standard of comparison for alternating direction methods.

3.2 The Alternating Direction Checkerboard Method and a New Formulation of the Frequency Transform

3.2a The Checkerboard Matrix Splitting

Checkerboard methods treat diffusion terms fuily implicitly for half the spatial points in a reactor and fully explicitly for the other half. To understand how this is done, consider the reactor mesh grid illustrated in Figure 3.2 in which every other mesh point is marked by an $\mathrm{x}$ and the remaining mesh points are marked with an 0 . 


\section{FIGURE 3.2}

Checkerboard Reactor Mesh Grid

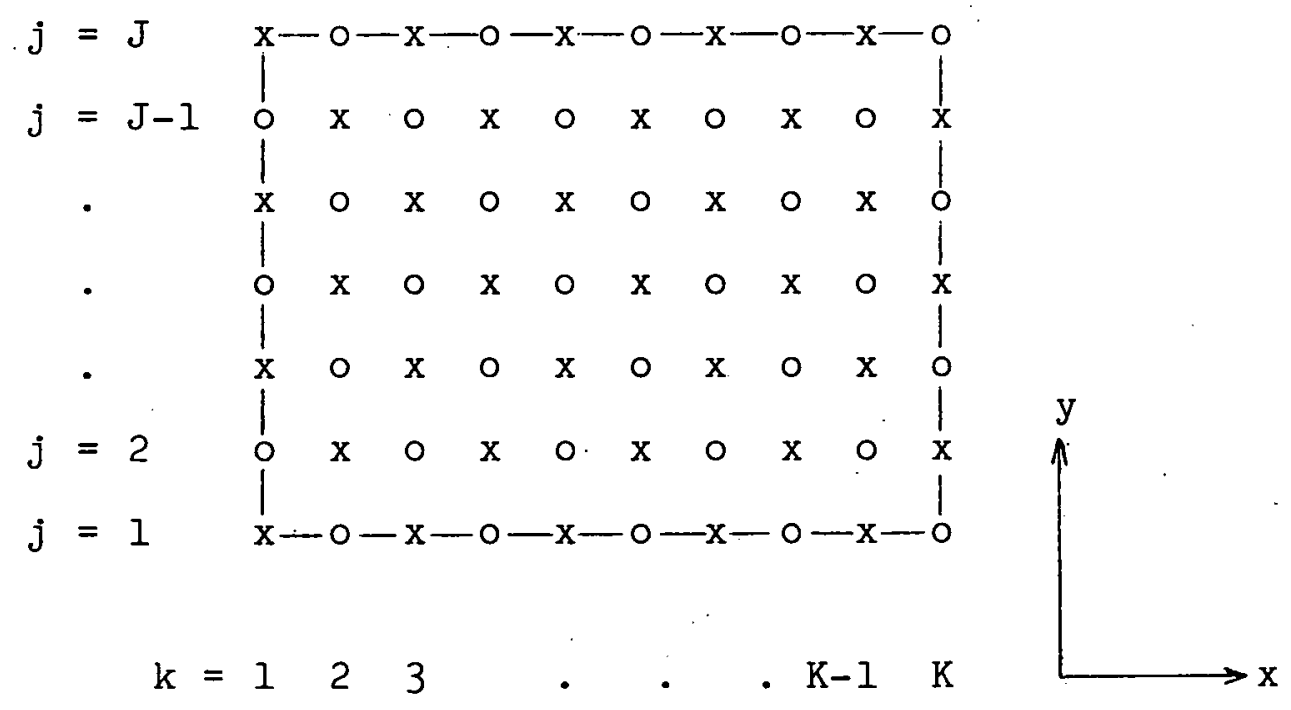


To solve for the flux at time $t^{n+l}$, diffusion at each $\mathrm{x}$-point in the first half-step is treated fully explicitly:

$\left.D_{g} \nabla^{2} \phi_{x g}\left(\bar{r}_{k_{i} j}, t\right)\right|_{t^{n}} ^{t^{n+1}}$

$=D_{g}\left[\frac{\phi_{o_{g, k+1, j}}^{n}-2 \phi_{x_{g, k, j}}^{n}+\phi_{o_{g, k-1, j}}^{n}}{h_{x}^{2}}+\frac{\phi_{o g, k, j+1}^{n}-2 \phi_{x, k, j}^{n}+\phi_{o g_{, k, j-1}}^{n}}{h_{y}^{2}}\right]$

The $x$-subscripts denote $x$-points and the o-subscripts denote. o-points. All the fluxes on the right hand side of Eq. (3.15) are known quantities. The first half-step is completed by determining the fluxes at time $t^{\mathrm{n}+\mathrm{l}}$ for the o-points. For this calculation, diffusion is treated fully implicitly:

$$
\begin{aligned}
& \left.D_{g} \nabla^{2} \phi_{o g}\left(\bar{r}_{k, j}, t\right)\right|_{t^{n}} ^{t^{n+1}} \ldots \\
& =D_{g}\left[\frac{\phi_{x, k+1, j}^{n+1}-2 \phi_{o g, k, j}^{n+1}+\phi_{x_{g, k-1, j}}^{n+1}}{h_{x}^{2}}+\frac{\phi_{x_{g, k, j+1}}^{n+1}-2 \phi_{o g, k, j}^{n+1}+\phi_{x g, k, j-1}^{n+1}}{h_{y}^{2}}\right]
\end{aligned}
$$


The fluxes $\phi_{x g, k+1, j}^{n+1}, \phi_{x g, k-1, j}^{n+1}, \phi_{x_{g, k, j+1}}^{n+1}$, and $\phi_{x g, k, j-1}^{n+1}$ of Eq. (3.16) are already known from the $x$-point calculation. Hence only $\phi_{\circ g, k, j}$ is unknown. It is as if all the fluxes were solved explicitly. Neither matrix inversions nor auxiliary storage is required.

For the second half-step the process is alternated; diffusion is treated explicitly for the o-points and then implicitly for the $\mathrm{x}$-points. The reactor mesh grid may be imagined as a checkerboard consisting of red and black squares where the orientation or direction of the checkerboard is changed at each half-step so that from half-step to half-step it becomes an alternating direction checkerboard. Note that the mesh points are not swept in any particular order. In effect, for each half-step all the $x$-points are solved simultaneously and then all the o-points are solved simultaneously. The nonsymmetrical buildup and cancellation of errors (Section 3.1d) observed in ADE methods as the spatial mesh grid is swept from one corner to another is not present in checkerboard methods.

The matrix form of checkerboard methods is found by dividing the A-matrix as before into

$$
\underline{A}=\underline{D}+\underline{R}
$$

All terms associated with diffusion are in $\underline{D}$. For consistency; assume that the diagonal terms associated with the precursors are also in $\underline{D}$ as before. All remaining terms 
of $\underline{A}$ are then in $\underline{R}$. In the checkerboard method the $\underline{D}$ matrix is split as

$$
\underline{D}=\underline{I}_{x} \underline{D}+\underline{I}_{0} \underline{D}
$$

where $I_{x}$ and $I_{0}$ are diagonal matrices of ones and zeros. Necessarily, $\underline{I}_{x}+\underline{I}_{0}=\underline{I}$, the identity matrix, and $\underline{I}_{X} \cdot \underline{I}_{0}=\underline{0}$, the null matrix. The placement of ones and zeros in the matrices $I_{x}$ and $I_{0}$ is such the diffusion is treated as described by Eqs. (3.15) and (3.16). Furthermore, let

$$
\underline{R}=\underline{R}_{1}+\underline{R}_{2}=\underline{R}_{3}+\underline{R}_{4}
$$

Then the checkerboard matrix splitting is

$$
\begin{array}{lll}
\underline{A}_{1}=\underline{I}_{x} \underline{D}+\underline{R}_{1} & ; & \underline{A}_{3}=\underline{I}_{0} \underline{D}+\underline{R}_{3} \\
\underline{A}_{2}=\underline{I}_{0} \underline{D}+\underline{R}_{2} & ; & \underline{A}_{4}=\underline{I}_{x} \underline{D}+\underline{R}_{4}
\end{array}
$$

There are many ways to choose $\underline{R}_{1}, \underline{R}_{2}, \underline{R}_{3}$ and $\underline{R}_{4}$; these alternate checkerboard methods will be described in section 3.2e. For now, consider the symmetric choice

$$
\begin{aligned}
& \underline{R}_{1}=\underline{R}_{4}=\underline{I}_{x} \underline{R} \\
& \underline{R}_{2}=\underline{R}_{3}=\underline{I}_{0} \underline{R}
\end{aligned}
$$

Here all terms at $x$ - and o-points are treated either fully explicitly or fully implicitly. This technique for partial differential equations is the idea of Gourlay 25 and was 
first proposed for reactor physics by werner ${ }^{17}$. As implemented in this thesis, Eq. (3.17) is called the alternating direction checkerboard (ADC) method.

\section{2b The Two-Level Frequency Transform}

The MITKIN frequency transform, Eq. (3.8), is incompatible with checkerboard methods. If frequencies for all points are computed by Eq. (3.9), then the frequencies at all $x$-points differ noticeably from the o-point frequencies when the checkerboard splitting is used. The transformation of variables, Eq. (3.4), then leads to one set of transformed fluxes for the $x$-points and another set for the o-points. Diffusion between these differently transformed variables can lead to errors.

A new formulation of the frequency transform equations is required for checkerboard methods. In this new formulation the exponential terms of Eq. (3.5) are not evaluated at a single time level midway through the time interval as before; instead, the exponential terms are evaluated at the same time levels as the coefficient transformed flux vectors, $\bar{\phi}^{n}, \bar{\phi}^{n+1}$ and $\bar{\phi}^{n+2}$. That is, the exponential terms are evaluated at $t=t^{n}, t=t^{n+1}$, and $t=t^{n+2}$ so that Eq. (3.5) becomes

$\frac{\bar{\phi}^{n+1}-\bar{\Phi}^{n}}{h}=e^{-\Omega t^{n}}\left(\underline{A}_{1}-\gamma \Omega\right) e^{\Omega t^{n}} \bar{\phi}^{n}+e^{-\Omega t^{n+1}}\left(\underline{A}_{2}-\alpha \Omega\right) e^{\Omega t^{n+1}} \bar{\phi}^{n+1}$ 


$$
\frac{\bar{\phi}^{n+2}-\bar{\Phi}^{n+1}}{h}=e^{-\Omega t^{n+1}}\left(A_{3}-\gamma \Omega\right) e^{\Omega t^{n+1}} \Phi^{n+1}+e^{-\Omega t^{n+2}}\left(A_{4}-\alpha \Omega\right) e^{\Omega t^{n+2}} \Phi^{n+2}
$$

where $\alpha+\gamma=1.0$

and as before, $\bar{\phi}$ is the transformed flux vector.

This will hereafter be called a two-level frequency transform because the frequencies are evaluated at two time levels for each half-step. The frequency transform of MITKIN may be called a single-level frequency transform. The two-level frequency transform equations may be expressed in terms of $\bar{\psi}$ by first combining Eqs. (3.18a) and $(3.18 b)$ :

$$
\begin{aligned}
\bar{\phi}^{n+2}= & e^{-\Omega t^{n+2}}\left[I-h\left(\underline{A}_{4}-\alpha \Omega\right)\right]^{-1} e^{\Omega t^{n+2}} e^{-\Omega t^{n+1}}\left[I+h\left(\underline{A}_{3}-\gamma \Omega\right)\right] e^{\Omega t^{n+1}} \\
& \times e^{-\underline{\Omega} t^{n+1}}\left[I-h\left(A_{2}-\alpha \Omega\right)\right]^{-1} e^{\Omega t^{n+1}} e^{-\Omega t^{n}}\left[I+h\left(A_{1}-\gamma \Omega\right)\right] e^{\Omega t^{n}} \bar{\phi}^{n}
\end{aligned}
$$

The vectors $\bar{\phi}$ and $\bar{\psi}$ are again related by

$\bar{\psi}^{n+2}=e^{\Omega t^{n+2}} \bar{\phi}^{n+2} \quad \because \quad \bar{\phi}^{n}=e^{-\Omega t^{n}} \bar{\psi}^{n}$

Inserting Eq. (3.7) into Eq. (3.19) leads to

$$
\begin{aligned}
\bar{\psi}^{n+2}= & {\left[I-h\left(\underline{A}_{4}-\alpha \underline{\Omega}\right)\right]^{-1} e^{\Omega h}\left[I+h\left(\underline{A}_{3}-\gamma \underline{\Omega}\right)\right] } \\
& \times\left[I-h\left(\underline{A}_{2}-\alpha \underline{\Omega}\right)\right]^{-1} e^{\Omega h}\left[I+h\left(\underline{A}_{1}-\gamma \Omega\right)\right] \Psi^{n}
\end{aligned}
$$


Since $\underline{A}_{1}, \underline{A}_{2}, \underline{A}_{3}$ and $\underline{A}_{4}$ are not specified, Eq. (3.20) is applicable to any alternating direction method.

Experimentation indicates that it is best to choose frequencies for the two-level frequency transform in the same way as for the single-level frequency transform, namely

$$
\Omega_{k, j}^{n+2}=\frac{1}{2 h} \ln \frac{\psi_{G, k, j}^{n}}{\psi_{G, k, j}^{n-2}}
$$

An acceptable alternative which is slightly faster makes use of the approximation

$$
\ln \frac{a}{b}=2 \frac{a-b}{a+b}
$$

in order to approximate Eq. (3.9) as

$$
\Omega_{k, j}^{n+2}=\frac{1}{h} \frac{\psi_{G, k, j}^{n}-\psi_{G, k, j}^{n-2}}{\psi_{G, k, j}^{n}+\psi_{G, k, j}^{n-2}}
$$

Also, it has been determined that a good choice of $\gamma$. and $\alpha$ is 


$$
\begin{aligned}
& \alpha=\gamma=1 / 2 \text { for symmetric methods } \\
& \alpha=1 ; \gamma=0 \text { for nonsymmetric methods }
\end{aligned}
$$

For checkerboard methods it is reasonable to choose $\alpha$ and $\gamma$. as the diagonal matrices $I_{x}$ and $I_{0}$. However, the choice $\alpha=\gamma=1 / 2$ has been found to be slightly better.

When the two-level frequency transform, Eq. (3.20), is applied to checkerboard methods, the problems of the single level transform, Eq. (3.8), are avoided. Diffusion at : explicit points is calculated with untransformed fluxes only; this is because the $e^{-2 h}$ terms in Eq. (3.20) are on the left hand sides of the $[\underline{I}+h(\underline{A},-\gamma \Omega)]$. and $\left[\underline{I}+h\left(\underline{A}_{3}-\gamma \Omega\right)\right]$ terms rather than on the right. For implicit points the transformation of Eq. (3.20) is such that all neighboring points are transformed consistently; thus diffusion at implicit points is also treated properly. Unlike the single-level frequency transform, the two-level frequency transform is compatible with all alternating direction methods.

The fact that the single-level frequency transform differs from the two-level frequency transform only by the placement of the $e^{\Omega h}$ terms suggests that other formulations of the frequency transform may be invented merely by shifting around the $e^{\Omega h}$ terms. The following two have been tried: 


$$
\begin{aligned}
\bar{\psi}^{n+2}= & e^{\Omega h}\left[\underline{I}-h\left(\underline{A}_{4}-\alpha \underline{\Omega}\right)\right]^{-1}\left[\underline{I}+h\left(\underline{A}_{3}-\gamma \underline{\Omega}\right)\right] \\
& \times e^{\Omega} \underline{h}\left[\underline{I}-h\left(\underline{A}_{2}-\alpha \underline{\Omega}\right)\right]^{-1}\left[\underline{I}+h\left(\underline{A}_{1}-\gamma \underline{\Omega}\right)\right] \bar{\psi}^{n}
\end{aligned}
$$

and

$$
\begin{aligned}
\bar{\psi}^{n+2}=e^{2} \underline{\Omega} h\left[\underline{I}-h\left(\underline{A}_{4}-\alpha \underline{\Omega}\right)\right]^{-1}\left[\underline{I}+h\left(\underline{A}_{3}-\gamma \underline{\Omega}\right)\right] \\
\times\left[\underline{I}-h\left(\underline{A}_{2}-\alpha \underline{\Omega}\right)\right]^{-1}\left[\underline{I}+h\left(\underline{A}_{1}-\gamma \underline{\Omega}\right)\right] \bar{\psi}^{n}
\end{aligned}
$$

Numerical testing indicates that these formulations are much less accurate than either the single-level or two-level frequency transforms.

\section{2c Two-Level Frequency Transform and Checkerboard Truncation Errors}

The truncation errors associated with the two-level frequency transform method differ somewhat from those of Section 3.1d which were associated with the single-level frequency transform. In order to determine the truncation errors, the expansion of Eq. (3.20)

$$
\begin{aligned}
\bar{\psi}^{n+2}=\left\{\underline{I}+2 h \underline{A}+h^{2}\left[\left(\underline{A}+\underline{A}_{2}+\underline{A}_{4}\right) \underline{A}+\Omega\left(\underline{A}-\underline{A}_{2}-\underline{A}_{4}\right)\right.\right. \\
\left.+(1-2 \alpha) \Omega(\underline{A}-\underline{\Omega})]+O\left(h^{3}\right)\right\} \bar{\psi}^{n}
\end{aligned}
$$

is compared to the true solution,

$$
\bar{\psi}^{n+2}=\left[\underline{I}+2 h \underline{A}+2 h^{2} \underline{A}^{2}+\frac{4}{3} h^{3} \underline{A}^{3}+O\left(h^{4}\right)\right] \bar{\psi}^{n}
$$

The two-level frequency transform truncation error is then 


$$
\begin{aligned}
T . E . & =\Psi_{\text {true }}^{n+2}-\Psi^{n+2} \\
& =\left\{h^{2}\left[\left(\underline{A}-\underline{A}_{2}-A_{4}\right) \underline{A}-\Omega\left(\underline{A}-\underline{A}_{2}-\underline{A}_{4}\right)-(1-2 \alpha) \Omega(A-\Omega)\right]+O\left(h^{3}\right)\right\} \bar{\psi}^{n}
\end{aligned}
$$

For symmetric alternating direction methods where

$$
\begin{aligned}
\alpha=\gamma & =1 / 2 \text { and } \underline{A}_{2}+\underline{A}_{4}=\underline{A}, \text { Eq. (3.20) becomes } \\
\Psi^{n+2}= & \left\{\underline{I}+2 h \underline{A}+2 h^{2} \underline{A}^{2}+h^{3}\left[2\left(\underline{A}^{2}-\underline{A}_{2} \underline{A}_{1}\right) \underline{A}+\frac{1}{2} \Omega^{2} \underline{A}+\underline{A}_{2} \Omega \underline{A}_{1}\right.\right. \\
& \left.\left.-\underline{A}_{2} \Omega \underline{A}_{2}-\underline{\Omega} \underline{A}_{1} \underline{A}-\frac{1}{6} \Omega^{3}\right]+O\left(h^{4}\right)\right\} \Psi^{n} .
\end{aligned}
$$

For this the truncation error is

$$
\begin{aligned}
\text { T.E. }= & \Psi_{\text {true }}^{n+2}-\bar{\psi}^{n+2} \\
= & \left\{h ^ { 3 } \left[2\left(\underline{A}_{2} \underline{A}_{1}-\frac{1}{3} \underline{A}^{2}\right) \underline{A}-\frac{1}{2} \Omega^{2} \underline{A}-\underline{A}_{2} \Omega \underline{A}_{1}+\underline{A}_{2} \Omega \underline{A}_{2}\right.\right. \\
& \left.\left.+\Omega \underline{A}_{1} \underline{A}+\frac{1}{6} \Omega^{3}\right]+O\left(h^{4}\right)\right\} \bar{\psi}^{n}
\end{aligned}
$$

Comparing these equations to the corresponding single-level frequency transform truncation error equations indicates that the order of the truncation error is the same for both frequency transform formulations. It is not clear from these equations which of the two formulations has the smaller truncation error.

Examination of the truncation error equations for the special case of the ADC method with $\Omega=0$ (no frequency transform) indicates that the ADC method has a smaller truncation error than other symmetric alternating direction 
methods. For all symmetric alternating direction methods with $\Omega=0$ the truncation error is (from either Eq. (3.13)) or Eq. (3.23)):

$$
T . E .=\left\{2 h^{3}\left[\underline{A}_{2} \underline{A}_{1} A-\frac{1}{3} \underline{A}^{3}\right]+O\left(h^{4}\right)\right\} \bar{\psi}^{n}
$$

In Section 3.le it was hypothesized that the truncation error term for the summetric SADI and SADE methods is relatively large. In particular it may be argued that the term $\underline{A}_{2} \underline{A}_{1} A \bar{\psi}^{n}$ is large. For the $A D C$ method, the $\underline{A}_{2} \underline{A}_{1} \underline{A}^{n}$ term becomes $\underline{I}_{0} \underline{A I}_{x} \underline{A A}^{\mathrm{n}}=\left(\underline{I}_{0} \underline{A I}_{\mathrm{x}}\right) \underline{A}^{2} \bar{\psi}^{\mathrm{n}}$. This may be smaller than $\underline{A}_{2} \underline{A}_{1} \underline{A} \bar{\psi}^{\text {n }}$ of the SADI and SADE schemes because generally,

$$
\left.A \bar{\psi}^{n} \approx \frac{\partial \bar{\psi}}{\partial t}\right|_{t=t^{n}}>\left.\frac{\partial^{2} \bar{\psi}}{\partial t^{2}}\right|_{t=t^{n}} \approx A^{2} \bar{\psi}^{n}
$$

or.

$$
A \bar{\psi}^{n}>\underline{A}^{2} \bar{\psi}^{n}
$$

Therefore, it may be conjectured that 


$$
\begin{aligned}
& \left(\underline{A}_{2} \underline{A}_{1}-\frac{1}{3} \underline{A}^{2}\right) \underline{A} \bar{\psi}^{n}>\left(\underline{I}_{0} \underline{A} \underline{I}_{x}-\frac{1}{3} \underline{A}\right) \underline{A}^{2} \bar{\psi}^{n} \\
& \text { SADE, SADI }
\end{aligned}
$$

It will be shown numerically in Section 3.4 that this

argument appears to be correct: the ADC method has a smaller truncation error than even the best explicit and implicit methods when $\Omega=0$. When the frequency transform is applied, however, a similar analysis of truncation errors is inconclusive.

\section{2d Computational Considerations of the ADC Method} When the multigroup neutron diffusion equation is solved by a digital computer certain computational considerations such as roundoff errors, computer storage, and program execution time become important. These considerations may be method dependent.

For example, several computational characteristics have already been described in section 3.1. In the ADI method, tridiagonal matrix inversions and auxiliary computer storage are required for each line of fluxes; the associated data management also increases program execution time. The $A D E$ method is characterized by roundoff errors which build up as the reactor spatial mesh grid is swept from one corner to the other; these errors are then cancelled in the next half-step as the mesh is swept back in the opposite direction. 
Computational considerations associated with the frequency transform include auxiliary computer storage and increased program execution time.

The ADC method is characterized by four-computational characteristics. Three of these limit the applicability of the method. Two of these apply to all checkerboard methods.

First, in the ADC method a dense GxG matrix must be inverted at each spatial mesh point for every two halfsteps. This is a consequence of Eq. (3.17b):

$$
\begin{aligned}
& \underline{R}_{1}=\underline{R}_{4}=\underline{I}_{x} \underline{R} \\
& \underline{R}_{2}=\underline{R}_{3}=\underline{I}_{0} \underline{R}
\end{aligned}
$$

Because $\underline{R}_{1}, \underline{R}_{2}, \underline{R}_{3}$ and $\underline{R}_{4}$ are not triangular matrices as was the case for the SADI, SADE, NSADI, and NSADE methods, trivial forward substitution inversions are not possible. As a result, the $A D C$ method is not practical for manygroup problems because of the time required for matrix inversions. However, for few-group problems these inversions can be carried out rapidiy if an efficient inversion routine such as a crout ${ }^{26}$ reduction is used. A comparison of program execution times is presented in Table 3.1 ; it is seen that the GxG matrix inversion requirement is not a great limitation for few-group problems. 
TABLE 3.1

Relative Computing Times

\begin{tabular}{|c|c|c|c|}
\hline \multirow[b]{2}{*}{$\begin{array}{l}\text { number of } \\
\text { energy groups }\end{array}$} & \multicolumn{3}{|c|}{$\begin{array}{l}\text { Computer time }{ }^{a} \text { required per } \\
\text { problem time step (seconds) }\end{array}$} \\
\hline & $G=1$ & $G=2$ & $G=4$ \\
\hline \multicolumn{4}{|l|}{ Code: } \\
\hline NSADE* & .010 & .206 & .701 \\
\hline $\mathrm{NSADE}^{\mathrm{c}}$ & .015 & .310 & 1.007 \\
\hline$A D C *$ & .011 & $.138^{\mathrm{d}}$ & $\begin{array}{r}.845^{\mathrm{e}} \\
1.330^{\mathrm{f}}\end{array}$ \\
\hline$A D C$ & .015 & $.191^{d}$ & $1.092^{\mathrm{e}}$ \\
\hline
\end{tabular}

* $\Omega=0$; frequencies not calculated

a) IBM $360 / 65$ computer used

b) the physics and geometry of the test problems are described in Appendix D; the 1, 2, and 4 group problems are associated with critical configurations 3,4 and 5 of Appendix D, respectively.

c) same as MITKIN method

d) $2 \times 2$ matrix inversions are handled analytically

e) GxG inversigns for $G>2$ are carried out by a Crout Reduction 26 .

f) GXG inversions were carried out by a Gauss Reduction. Note that this is $50 \%$ slower than the Crout Reduction. 
Secondly, fast group neutron velocities are poorly handled by the ADC method. For each half-step either the $x$-points or the o-points of the reactor mesh grid are treated fully explicitly by the ADC method. In any fully explicit treatment of group-to-group transfer the roundoff errors of the fast group are magnified by a factor of $\mathrm{v}_{1} / \mathrm{v}_{\mathrm{G}}$ in the thermal group. Because $\mathrm{v}_{1}$ (the fast group neutron velocity) can be many: orders of magnitude larger than $v_{G}$ (the thermal group neutron velocity), the thermal group roundoff error may become intolerably large. For such problems it is necessary to reduce the fast group. velocities arbitrarily. This may be done by setting

$$
\mathrm{v}_{\mathrm{g}}=\mathrm{v}_{\mathrm{G}} \quad \mathrm{g}=1,2, \ldots, \mathrm{G}
$$

Generally the effects of such an adjustment are small.* Third, perturbations are inadequately spatially propagated by checkerboard methods. According to diffusion theory, perturbations anywhere in a reactor are "felt" immediately everywhere else in the reactor. For a numerical model, therefore, a perturbation at any mesh point should cause some effect at all other mesh points within at least two half-steps. This is the case for ADI and ADE methods.

* In Chapter 2 it is shown that the asymptotic solution of the diffusion equation is unaffected by the value of the neutron velocity. 
This is not the case for checkerboard methods; a perturbation propagates only one spatial mesh per temporal half-step. For example, suppose a reactor operating in the steady state is modeled by the reactor mesh grid of Figure 3.2. If the flux at mesh point $(J, K)$ is suddenly increased, diffusion theory predicts that the flux at mesh point $(1,1)$ will immediately increase in response. For the checkerboard model, diffusion is treated in such a fashion that the flux at $(1,1)$ will not immediately increase but rather will remain at its steady state value until after either $J$ or $K$ half-steps, whilchever is larger, have been taken. For reactor problems modeled by an extremely fine spatial mesh grid ( $h_{x}$ or $h_{y} \ll l$ neutron mean free path) a perturbation of the flux at one mesh point swiftly and significantly affects flux values at mesh points many mesh spaces away. For these problems checkerboard methods may be poor. However, coarser mesh problems are more common. For these problems a perturbation at one mesh point immediately affects only fluxes at neighboring mesh points. Flux values at mesh points many mean free paths away are only insignificantly perturbed. For the problems examined in this thesis perturbation propagation effects were not found to be significant.

A fourth computational characteristic of the ADC method is the incompatibility of checkerboard methods with the single-level frequency transform (Section 3.2b). However, 
this cannot be classed as a limitation because the checkerboard methods are compatible with the two-level frequency transform.

The computational characteristics of the ADC method are summarized in Table 3.2. Some of the problems that have been mentioned may be avoided by using alternative methods, some of which are described in the next section. $3.2 \mathrm{e}$ Alternative Checkerboard Methods

A number of checkerboard methods alternative to the ADC method have been tested numerically. These alternative checkerboard methods are either minor modifications of the ADC method or blendings of the ADC and NSADE method.

With minor alterations, the ADC code can be modified to solve the neutron diffusion equation by the following matrix splitting:

$$
\begin{aligned}
& \underline{A}_{1}=\underline{A}_{4}=\underline{I}_{x} \underline{D}+\underline{I}_{0} \underline{R} \\
& \underline{A}_{2}=\underline{A}_{3}=\underline{I}_{0} \underline{D}+\underline{I}_{x} \underline{R}
\end{aligned}
$$

Because the flux at any mesh point is no longer treated fully explicitly in either half-step, fast group neutron velocities need not be adjusted in order to avoid roundoff errors. However, numerical testing indicates that the. truncation error of this method is larger than that of the ADC method. Thus smaller time step sizes are required for a given accuracy. 
TABLE 3.2

ADC Computational Characteristics

1) GXG matrix inversions are required at each spatial mesh point every two half-steps for the ADC method;

2) When fast group neutron velocities are several orders of magnitude larger than thermal group neutron velocities they must b.e arbitrarily reduced in order to avoid excessive roundoff errors for the ADC method;

3) Perturbations are propagated only one spatial mesh at a time per half-step for all checkerboard methods;

4) Checkerboard methods are incompatible with the single-level frequency transformation. 
A generalization of Eq. (3.24a) is the following matrix splitting:

$$
\begin{aligned}
& \underline{A}_{1}+\underline{I}_{x} \underline{D}+\underline{I}_{0}(\underline{R}-\underline{S})+\underline{S}_{1} \\
& \underline{A}_{2}=\underline{I}_{0} \underline{D}+\underline{I}_{x}(\underline{R}-\underline{S})+\underline{S}_{2} \\
& \underline{A}_{3}=\underline{I}_{0} \underline{D}+\underline{I}_{x}(\underline{R}-\underline{S})+\underline{S}_{3} \\
& \underline{A}_{4}=\underline{I}_{x} \underline{D}+\underline{I}_{0}(\underline{R}-\underline{S})+\underline{S}_{4}
\end{aligned}
$$

where $\underline{s}_{1}+\underline{s}_{2}=\underline{s}_{3}+\underline{s}_{4}=\underline{s}$

and $\underline{S}$ is a diagonal matrix consisting of various diagonal components of $\underline{R}$. This splitting reduces to that of $\mathrm{Eq}$. (3.24a) if $\underline{S}=\underline{0}$, the null matrix. For other choices of $\underline{s}$ the method of Eq. (3.24b) can be made better than that of Eq. (3.24a). The best choice seems to be one where $\underline{S}_{2}$ and $\underline{s}_{4}$ each equals the diagonal matrix of removal cross section terms and where $\underline{S}_{1}$ and $\underline{S}_{3}$ each contains the remaining diagonal elements of R. But regardless of the choice of $\underline{S}_{1}, \underline{S}_{2}, \underline{S}_{3}$; and $\underline{S}_{4}$, undesirably small time step sizes are still required and hence the methods of Eqs. (3.24a) and (3.24b) are poor. Another alternative to the ADC method and an obvious way to avoid GXG matrix. inversions is to choose the matrices $\underline{R}_{1}, \underline{R}_{2}, \underline{R}_{3}$ and $\underline{R}_{4}$ of Eq. (3.17a) to be the same as $\underline{R}_{1}, \underline{R}_{2}$, $\underline{R}_{3}$ and $\underline{R}_{4}$ of the NSADE method. These matrices are then all triangular and may be simply inverted. The checkerboard method using this choice can be called the NSADC method. 
The NSADC formulation is identical to that of the NSADE with the exception of the diffusion matrices:

$$
\begin{aligned}
& \underline{D}=\underline{D}_{1}+\underline{D}_{2} \text { for NSADE } \\
& \underline{D}=\underline{I}_{x} \underline{D}+\underline{I}_{0} \underline{D} \text { for NSADC }
\end{aligned}
$$

As a result of this similarity, the $O\left(h^{2}\right)$ truncation error term of both methods is identical; the truncation errors only differ for higher order terms. Furthermore, the flux at $\mathrm{x}$ - and $\mathrm{o}-$ mesh points is treated neither fully implicitly nor fully explicitly as in the ADC method. Therefore, fast group velocities need not be modified. Only the third and fourth computational characteristics (Table 3.2 ) of the ADC method apply to the NSADC method. Solutions from the NSADC method are compared to those of other methods in Section 3.4 .

A final alternative method makes use of the splitting

$$
\begin{aligned}
& \underline{A}_{1}=\underline{A}_{4}=\underline{D}_{1}+\underline{I}_{x} \underline{R} \\
& \underline{A}_{2}=\underline{A}_{3}=\underline{D}_{2}+\underline{I}_{0} \underline{R}
\end{aligned}
$$

where $\underline{D}_{1}, \underline{D}_{2}$ and $\underline{R}$ are defined as in Section 3.1d. This may be described as an ADE method with intragroup transfer handled in a checkerboard fashion. Hence this symmetric checkerboard ADE method will be referred to as the SCADE method. Of the limitations listed in Table 3.1, only the GXG matrix inversion requirement applies. Unlike the SADE method, the energy mesh is not undesirably swept in the 
direction of loose coupling. Furthermore, the number of GXG matrix inversions required for the SCADE method is only half the number required for the SIADE method of Eq. (3.14). Numerical results from this splitting method are also presented in Section 3.4 .

\subsection{Numerical Properties}

Approximate methods for solving the neutron diffusion equation should have the following numerical properties:

1) the temporal truncation error should be small;

2.) The steady state behavior of the approximate solution should equal that of the true solution;

3) the approximate solution should converge to that of the true solution as the time step size is decreased. of these properties, truncation errors have been discussed in previous sections. The steady state behavior of all alternating direction methods is analyzed in section 3.3a. According to the famous Lax Theorem 27 the convergence requirement is equivalent to requiring numerical consistency and stability. Consistency is proven for all alternating direction methods in section 3.3b. Stability is proven for a wide class of alternating direction methods in section 3.3c. These general proofs are also applicable to the theta methods described in Chapters 1 and 2 . 3.3a Steady State Behavior

All alternating direction methods have the same steady. state behavior as the true solution of the multigroup neu- 
115

tron diffusion equation. That is, $\bar{\psi}^{n+2}=\bar{\psi}^{n}$ for all n.

Proof:

In the steady state, $\frac{\partial \bar{\psi}}{\partial t}=0$. Hence $\underline{\bar{\psi}}=\underline{\mathrm{A}} \bar{\psi}^{n}=0$ and $\Omega=0$. Because $\Omega=0$,

$\bar{\psi}^{n+2}=\left[I-h A_{4}\right]^{-1}\left[I+h \underline{A}_{3}\right]\left[I-h A_{2}\right]^{-1}\left[I+h \underline{A}_{1}\right] \bar{\psi}^{n}$

for alternating direction methods regardless of which irequincy transform method, if any, is used.

Therefore,

$$
\begin{aligned}
\psi^{n+2} & =\left[I-h \underline{A}_{4}\right]^{-1}\left[I+h \underline{A}_{3}\right]\left[I-h \underline{A}_{2}\right]^{-1}\left[I+h \underline{A}_{1}\right] \bar{\psi}^{n} \\
& =\left[I-h \underline{A}_{4}\right]^{-1}\left[I+h\left(\underline{A}-\underline{A}_{4}\right)\right]\left[\underline{I}-h \underline{A}_{2}\right]^{-1}\left[I+h\left(\underline{A}-\underline{A}_{2}\right)\right] \bar{\psi}^{n} \\
& =\left[\underline{I}-h \underline{A}_{4}\right]^{-1}\left[I+h\left(\underline{A}-\underline{A}_{4}\right)\right]\left[\underline{I}-h \underline{A}_{2}\right]^{-1}\left[\underline{I}-h \underline{A}_{2}\right] \bar{\psi}^{n} \\
& =\left[\underline{I}-h \underline{A}_{4}\right]^{-1}\left[I+h\left(\underline{A}-\underline{A}_{4}\right)\right] \bar{\psi}^{n} \\
& =\left[\underline{I}-h \underline{A}_{4}\right]^{-1}\left[\underline{I}-h \underline{A}_{4}\right] \bar{\psi}^{n} \\
& =\tilde{\psi}^{n}
\end{aligned}
$$




\section{3b Consistency}

A finite difference method is consistent if the finite difference solution approaches the differential equation solution as the time step size, $h$, approaches zero.

The neutron diffusion equation may be written in differential operator form as in Eq. (1.3a) of Section 1.2:

$$
\frac{\partial \bar{\Phi}(r, t)}{\partial t}=\underline{a} \bar{\Phi}(r, t)
$$

The differential equation solution, $\Phi\left(\bar{r}, t^{n+2}\right)$, at time $t^{n+2}$ cannot be compared with the alternating direction method finite difference solution, $\bar{\psi}^{n+2}$, at time $t^{n+2}$. This is because the true solution vector, $\bar{\Phi}\left(\bar{r}, t^{n+2}\right)$, is continuous in space and has fewer elements than the vector $\bar{\psi}^{n+2}$. However, it is possible to construct a discretized true solution vector.

Suppose $\Psi^{n+2}$ is a $(G+I)^{*} N$ element vector in which $G$ is the number of energy groups, I is the number of precursor groups, and $\mathrm{N}$ is the number of spatial mesh points. Let the $(G+I)$ element vector $\bar{\Phi}(\bar{r}, t)$ be evaluated at each of the $\mathrm{N}$ spatial mesh points, $\overline{\mathrm{r}}_{\mathrm{n}}$. Then combine these $\mathrm{N}$ vectors into one as 
$\bar{\theta} \equiv\left[\begin{array}{c}\bar{\Phi}\left(\bar{r}_{1}, t\right) \\ \bar{\Phi}\left(\bar{r}_{2}, t\right) \\ \vdots \\ \bar{\Phi}\left(\bar{r}_{n}, t\right) \\ \vdots \\ \bar{\Phi}\left(\bar{r}_{N}, t\right)\end{array}\right]$.

Furthermore, assume that there is a number matrix, $\underline{M}$, such that

$$
\frac{\partial \bar{\theta}}{\partial t}=\underline{M} \bar{\theta}
$$

It is not necessary to specify how the diffusion operators of $Q$ are represented in the matrix $\underline{M}$. What is important is that Eq. (3.25) gives (by definition) the exact solution of the (differential) neutron diffusion equation and that $\bar{\theta}$ and $\underline{M}$ have the same dimensions as $\bar{\psi}$ and $\underline{A}$ of the finite difference equations.

It will now be shown that any finite difference method for which 


$$
\bar{\psi}^{n+2}=\underline{B} \bar{\psi}^{n}
$$

where $\underline{B}=\left[\underline{I}+2 h \underline{A}+O\left(h^{2}\right)\right]$

is consistent if $\underline{A}$ is a second order approximation to $\underline{M}$ and if the ratios

$$
r_{x}=\frac{h}{h_{x}^{2}} \quad ; \quad r_{y}=\frac{h}{h_{y}^{2}} \quad \text { etc. }
$$

are fixed.

Proof:

The finite difference matrix, $A$, is a second order approximation to $\underline{\mathrm{M}}$ :

$$
\underline{A}=\underline{M}+O\left(h_{x}^{2}\right)+O\left(h_{y}^{2}\right)
$$

This is because diffusion is treated by central difference approximations 9 in the finite difference matrix, $\underline{A}$. No such approximation is made in the matrix, $\underline{M}$, which yields the true differential equation solution at each mesh point. 
Also,

$$
\bar{\psi}^{n+2}=\left[\underline{I}+2 h A+O\left(h^{2}\right)\right] \bar{\psi}^{n}
$$

This has been demonstrated in previous sections for all alternating direction methods with and without either frequency transform.

Finally,

$$
\bar{\theta}^{n+2}=\left[\underline{I}+2 h \underline{M}+O\left(h^{2}\right)\right] \bar{\theta}^{n} .
$$

This is merely the Taylor Series expansion of the true solution vector, $\bar{\theta}^{n+2}$.

The consistency requirement is that the approximate solution, $\bar{\psi}^{n+2}$, approaches the true solution, $\bar{\theta}^{n+2}$, as $\mathrm{h} \rightarrow 0$. That is,

$$
\lim _{h \rightarrow 0} \bar{\psi}^{n+2}=\bar{\theta}^{n+2}
$$

A more formal consistency requirement is stated in terms of vector norms:

$$
\lim _{h \rightarrow 0}\left\|\frac{\bar{\psi}^{n+2}-\bar{\psi}^{n}}{2 h}\right\|=\lim _{h \rightarrow 0}\left\|\frac{\bar{\theta}^{n+2}-\bar{\theta}^{n}}{2 h}\right\|=\left.\frac{\partial \bar{\theta}}{\partial t}\right|_{t=t^{n}}
$$

(Vector and matrix norms are reviewed in Appendix B.) 
Assuming that the flux at time $t^{n}$ is known, $\bar{\psi}^{n}=\bar{\theta}^{n}$, the consistency requirement may be recast as

$$
\lim _{h \rightarrow 0}\left\|\frac{\bar{\psi}^{n+2}-\bar{\theta}^{n}}{2 h}\right\|=\lim _{h \rightarrow 0}\left\|\frac{\bar{\theta}^{n+2}-\bar{\theta}^{n}}{2 h}\right\|
$$

or alternatively,

$$
\lim _{h \rightarrow 0}\left\|\frac{\bar{\psi}^{n+2}-\bar{\theta}^{n+2}}{2 h}\right\|=0
$$

Now,

$$
\begin{aligned}
& \left\|\frac{\bar{\psi}^{n+2}-\bar{\theta}^{n+2}}{2 h}\right\| \\
& \quad=\left\|\frac{\left[\underline{I}+2 h \underline{\theta}+O\left(h^{2}\right)\right] \bar{\theta}^{n}-\left[\underline{I}+2 \dot{h} \underline{M}+O\left(h^{2}\right)\right] \bar{\theta}^{n} \|}{2 h}\right\| \\
& =\left\|\left[\underline{M}+O\left(h_{x}^{2}\right)+O\left(h_{y}^{2}\right)-\underline{M}+O(h)\right] \bar{\theta}^{n}\right\|
\end{aligned}
$$

Because the ratios $r_{x}=h / h_{x}^{2}$ and $r_{y}=h / h_{y}^{2}$ are fixed, $h_{x}^{2}$ and $h_{y}^{2}$ approach zero as $h$ approaches zero. Therefore, 
$\lim _{h \rightarrow 0}\left\|\frac{\bar{\psi}^{n+2}-\bar{\theta}^{n+2} \|}{2 h}\right\|$

$=\lim _{h \rightarrow 0}\left\|\left[M+O\left(h_{x}^{2}\right)+O\left(h_{y}^{2}\right)-\underline{M}+O(h)\right] \bar{\theta}^{n}\right\|$

$=0$

Q.E.D.

\section{3c Stability}

The ADI, ADE, and checkerboard methods are all unconditionally stable.. To prove this, some theorems are necessary.

Theorem 1: 28

If $\left(\underline{C}+\underline{c}^{T}\right)$ is real and positive definite, then $(\underline{I}-h \underline{C})(\underline{I}+h \underline{C})^{-1}$ is a bounded operator such that

$$
\left\|(I-h \underline{C})(I+h \underline{C})^{-1}\right\|_{2} \leqslant 1
$$

Proof:

Let $\underline{T}=(\underline{I}-h \underline{C})(\underline{I}+h \underline{C})^{-1}$ 
122

Let $\bar{\mu}$ be the real nonzero vector for which $\frac{(T \bar{u})^{\top}(T \bar{u})}{\bar{u}^{\top} \bar{u}}$ is maximized.

Let $\bar{v} \equiv(\underline{I}+h \underline{C})^{-1} \bar{u} \quad$; hence $\bar{u}=(\underline{I}+h \underline{C}) \bar{v}$. and $\bar{I} \bar{u}=(\underline{I}-h \subseteq)(\underline{I}+h \underline{C})^{-1} \bar{u}=(\underline{I}-h \underline{C}) \bar{v}$

Note that $\bar{\nabla}^{\top}\left(\underline{C}^{\top}+\underline{C}\right) \bar{v} \geqslant 0$ : because $\left(\underline{C}^{\top}+\underline{C}\right)$ is positive definite. Also, $\bar{v}^{\top} \bar{\nabla} \geqslant 0$ and $(\underline{C} \bar{v})^{\top}(\underline{C} \bar{v}) \geqslant 0$ because the product of a real vector and its transpose is always a positive number. Therefore,

$$
\begin{aligned}
&\left\|(\underline{I}-h \underline{C})(\underline{I}+h \underline{C})^{-1}\right\|_{2} \equiv\left\{\frac{(\underline{\underline{T}} \bar{u})^{\top}(\underline{\underline{T}} \bar{u})}{\bar{u}^{\top} \bar{u}}\right\}^{1 / 2} \\
&=\left\{\frac{[(\underline{I}-h \underline{C}) \bar{v}]^{\top}[(\underline{I}-h \underline{C}) \bar{v}]}{\left[(\underline{I}+h \underline{C}) \bar{v}^{\top}[(\underline{I}+h \underline{C}) \bar{v}]\right.}\right\}^{1 / 2} \\
&=\left\{\frac{\bar{v}^{\top} \bar{v}-h \bar{v}^{\top} \underline{C}^{\top} \bar{v}-h \bar{v}^{\top} \underline{C} \bar{v}+h^{2}(\underline{C} \bar{v})^{\top}(\underline{C} \bar{v})}{\bar{v}^{\top} \bar{v}+h \bar{v}^{\top} \underline{C}^{\top} \bar{v}+h \bar{v}^{\top} \underline{C} \bar{v}+h^{2}(\underline{C} \bar{v})^{\top}(\underline{C} \bar{v})}\right\}^{1 / 2} \\
&=\left\{\frac{\bar{v}^{\top} \bar{v}-h \bar{v}^{\top}\left(\underline{C}^{\top}+\underline{C}\right) \bar{v}+h^{2}(\underline{C} \bar{v})^{\top}(\underline{C} \bar{v})}{\bar{v}^{\top} \bar{v}+h \bar{v}^{\top}\left(\underline{C}^{\top}+\underline{C}\right) \bar{v}+h^{2}(\underline{C} \bar{v})^{\top}(\underline{C} \bar{v})}\right\}^{1 / 2} \\
& \leqslant 1
\end{aligned}
$$




\section{Theorem 2:}

Any system of equations

$$
\bar{\psi}^{n+2}=B \bar{\psi}^{n}
$$

or equivalently,

$$
\bar{\psi}^{2 m}=\underline{B}^{m} \bar{\psi}^{\circ}
$$

where

$$
B=\left[\underline{I}+h \underline{C}_{1}\right]^{-1}\left[\underline{I}-h \underline{C}_{2}\right]\left[\underline{I}+h \underline{C}_{2}\right]^{-1}\left[\underline{I}-h \underline{C}_{1}\right]
$$

is unconditionally stable if either of the following sets of conditions is met:

1). $\underline{\mathrm{C}}_{1}$ and $\underline{\mathrm{c}}_{2}$ are real and
a) $\left(\underline{C}_{1}+\underline{c}_{1}^{\mathrm{T}}\right)$ and $\left(\underline{\mathrm{C}}_{2}+\underline{\mathrm{c}}_{2}^{\mathrm{T}}\right)$ are positive definite;
b) $\left\|\left(\underline{I}+h \underline{C}_{1}\right)^{-1}\right\|_{2}$ and $\left\|\left(\underline{I}+h \underline{C}_{1}\right)\right\|$ ire bounded;

or

c) $\underline{c}_{1}$ and $\underline{c}_{2}$ may be factored as

$$
\underline{\mathrm{C}}_{1}=\underline{\mathrm{F}}_{1} \underline{\mathrm{H}} ; \quad \underline{\mathrm{C}}_{2}=\underline{\mathrm{F}}_{2} \underline{\mathrm{H}}
$$

where 
124

a) $\underline{F}_{1}, \underline{F}_{2}$, and $\underline{H}$ are real, symmetric, and positive definite. Hence, these matrices have real square roots :

$$
\underline{F}_{1}=\underline{F}_{1}^{T / 2} \underline{F}_{1}^{1 / 2} ; \underline{F}_{2}=\underline{F}_{2}^{T / 2} \underline{F}_{2}^{1 / 2} ; \underline{H}=\underline{H}^{T / 2} \underline{H}^{1 / 2}
$$

b) $\left\|\left(\underline{I}+h \underline{F}_{1} \underline{H}\right)^{-1}\right\|_{2},\left\|\left(\underline{I}+h \underline{F}_{1} \underline{H}\right)\right\|_{2},\left\|\underline{H}^{1 / 2}\right\|_{2}$ and $\left\|\underline{H}^{-1 / 2}\right\|_{2}$ are all bounded.

Proof:

$$
\begin{aligned}
\underline{B}= & \left(\underline{I}+h \underline{C}_{1}\right)^{-1}\left(\underline{I}-h \underline{C}_{2}\right)\left(\underline{I}+h \underline{C}_{2}\right)^{-1}\left(\underline{I}-h \underline{C}_{1}\right) \\
= & \left(\underline{I}+h \underline{C}_{1}\right)^{-1}\left(\underline{I}-h \underline{C}_{2}\right)\left(\underline{I}+h \underline{C}_{2}\right)^{-1} \\
& \times\left(\underline{I}-h \underline{C}_{1}\right)\left(\underline{I}+h \underline{C}_{1}\right)^{-1}\left(\underline{I}+h \underline{C}_{1}\right) \\
= & \left(\underline{I}+h \underline{C}_{1}\right)\left[\left(\underline{I}-h \underline{C}_{2}\right)\left(\underline{I}+h \underline{C}_{2}\right)^{-1}\right] \\
& \times\left[\left(\underline{I}-h \underline{C}_{1}\right)\left(\underline{I}+h \underline{C}_{1}\right)^{-1}\right]\left(\underline{I}+h \underline{C}_{1}\right) \\
= & \left(\underline{I}+h \underline{C}_{1}\right) \underline{E}_{2} \underline{E}_{1}\left(\underline{I}+h \underline{C}_{1}\right)
\end{aligned}
$$

where

$$
\begin{aligned}
& \underline{E}_{1} \equiv\left[\left(I-h \underline{C}_{1}\right)\left(I+h \underline{C}_{1}\right)^{-1}\right] \\
& \underline{E}_{2} \equiv\left[\left(I-h \underline{C}_{2}\right)\left(I+h \underline{C}_{2}\right)^{-1}\right]
\end{aligned}
$$

or

$$
\underline{E}_{k} \equiv\left[\left(I-h \underline{C}_{k}\right)\left(\underline{I}+h \underline{C}_{k}\right)^{-1}\right] . \quad k=1,2
$$


125

Thus

$$
\begin{aligned}
\underline{B}^{m} & =\left[\left(\underline{I}+h \underline{C}_{1}\right)^{-1} \underline{E}_{2} \underline{E}_{1}\left(\underline{I}+h \underline{C}_{1}\right)\right]^{m} \\
& =\left(\underline{I}+h \underline{C}_{1}\right)^{-1}\left[\underline{E}_{2} \underline{E}_{1}\right]^{m}\left(\underline{I}+h \underline{C}_{1}\right)
\end{aligned}
$$

Now if $\left(\underline{C}_{K}+\underline{C}_{K}^{T}\right)$ is positive definite then

$$
\left\|E_{k}\right\|_{2} \leq 1
$$

by Theorem 1 .

Thus

$$
\begin{aligned}
\left\|\left(E_{2} E_{1}\right)^{m}\right\|_{2} & \leq\left\|E_{2} E_{1}\right\|_{2}^{m} \\
& \leq\left\|E_{2}\right\|_{2}^{m} \cdot\left\|E_{1}\right\|_{2}^{m} \leq 1
\end{aligned}
$$

Therefore, from Eq. (3.26),

$$
\begin{aligned}
& \left\|\underline{B}^{m}\right\|_{2}=\left\|\left(\underline{I}+h \underline{C}_{1}\right)^{-1}\left[\underline{E}_{2} \underline{E}_{1}\right]^{m}\left(\underline{I}+h \underline{C}_{1}\right)\right\|_{2} \\
& \leq\left\|\left(I+h \underline{C}_{1}\right)^{-1}\right\|_{2}\left\|\left(\underline{E}_{2} \underline{E}_{1}\right)^{m}\right\|_{2}\left\|\left(\underline{I}+h \underline{C}_{1}\right)\right\|_{2} \\
& \leq\left\|\left(\underline{I}+h \underline{C}_{1}\right)^{-1}\right\|_{2}\left\|\left(\underline{I}+h \underline{C}_{1}\right)\right\|_{2} \\
& \leqslant K_{p} \text { a constant }
\end{aligned}
$$


If $\left(\underline{C}_{k}+\underline{C}_{k}^{\top}\right)$ is not positive definite but $\underline{C}_{k}=\underline{F}_{k} \underline{H}$ where $F_{k}$ and $\underline{H}$ are such that

$$
\underline{F}_{k}=\underline{F}_{k}^{T / 2} \underline{F}_{k} ; \quad \underline{H}=\underline{H}^{T / 2} \underline{H}
$$

then stability is proven as follows:

$$
\begin{aligned}
\underline{E}_{k} & =\left[\left(\underline{I}-h \underline{C}_{k}\right)\left(\underline{I}+h \underline{C}_{k}\right)^{-1}\right] \\
& =\left(\underline{I}-h \underline{F}_{k} \underline{H}\right)\left(\underline{I}+h \underline{F}_{k} \underline{H}\right)^{-1} \\
& =\left[\underline{H}^{-1 / 2}\left(\underline{I}-h \underline{H}^{1 / 2} \underline{F}_{k} \underline{H}^{T / 2}\right) \underline{H}^{1 / 2}\right]\left[\underline{H}^{-1 / 2}\left(\underline{I}+h \underline{H}^{1 / 2} \underline{F}_{k} \underline{H}^{T / 2}\right) \underline{H}^{1 / 2}\right]^{-1} \\
& =\underline{H}^{-1 / 2}\left(\underline{I}-h \underline{H}^{1 / 2} \underline{F}_{k} \underline{H}^{T / 2}\right)\left(\underline{I}+h \underline{H}^{1 / 2} \underline{F}_{k} \underline{H}^{T / 2}\right)^{-1} \underline{H}^{1 / 2}
\end{aligned}
$$

Let

then

$$
\underline{G}_{K} \equiv\left(\underline{I}-h \underline{H}^{1 / 2} \underline{E}_{K} \underline{H}^{T / 2}\right)\left(\underline{I}+h \underline{H}^{1 / 2} \underline{E}_{K} \underline{H}^{T / 2}\right)^{-1}
$$

and

$$
\underline{E}_{k}=\underline{H}^{-1 / 2} \underline{G}_{k} \underline{H}^{1 / 2}
$$

$$
\begin{aligned}
\left(\underline{E}_{2} \underline{E}_{1}\right)^{m} & =\left(\underline{H}^{-1 / 2} \underline{G}_{2} \underline{G}_{1} \underline{H}^{1 / 2}\right)^{m} \\
& =\underline{H}^{-1 / 2}\left(\underline{G}_{2} \underline{G}_{1}\right)^{m} \underline{H}^{1 / 2}
\end{aligned}
$$

In order to determine the norm of $\underline{G}_{k}$, let

$$
\begin{aligned}
P_{k} & \equiv \underline{H}^{1 / 2} \underline{E}_{k} \underline{H}^{T / 2} \\
& =\underline{H}^{1 / 2} \cdot E_{k}^{T / 2} \underline{F}_{k}^{1 / 2} \underline{H}^{T / 2}
\end{aligned}
$$




$$
\begin{aligned}
\underline{P}_{k} & =\left(\underline{F}_{k}^{1 / 2} \underline{H}^{T / 2}\right)^{\top}\left(\underline{F}_{K}^{1 / 2} \underline{H}^{T / 2}\right) \\
& =\underline{P}_{K}^{\top}
\end{aligned}
$$

Then for any real vector $\bar{u}$,

$$
\begin{aligned}
\bar{u}^{\top}\left(\underline{P}_{K}+\underline{P}_{k}^{\top}\right) \bar{u} & =2 \bar{u}^{\top} \underline{P}_{k} \bar{u} \\
& =2 \bar{u}^{\top}\left(\underline{E}_{k}^{1 / 2} \underline{H}^{T / 2}\right)^{\top}\left(\underline{E}_{k}^{1 / 2} \underline{H}^{T / 2}\right) \bar{u} \\
& =2\left(\underline{E}_{k}^{1 / 2} \underline{H}^{T / 2} \bar{u}\right)^{\top}\left(\underline{E}_{k}^{1 / 2} \underline{H}^{T / 2} \bar{u}\right) \\
& =\text { product of vector and its transpose } \\
\geqslant & 0
\end{aligned}
$$

Now, because $\bar{u}^{\top}\left(\underline{P}_{k}+\underline{P}_{k}^{\top}\right) \bar{u} \geqslant 0$

$\left(\underline{P}_{k}+\underline{P}_{k}^{\top}\right)$ must be positive definite. Thus

$$
\left\|\underline{G}_{k}\right\|_{2}=\left\|\left(I-h \underline{P}_{k}\right)\left(\underline{I}+h \underline{P}_{k}\right)^{-1}\right\|_{2} \leqslant 1
$$

by Theorem 1. Therefore,

$$
\left\|\left(\underline{G}_{2} \underline{G}_{1}\right)^{m}\right\|_{2} \leqslant\left\|\underline{G}_{2}\right\|_{2}^{m}\left\|\underline{G}_{1}\right\|_{2}^{m} \leqslant 1
$$

Finally, from Eqs. (3.26) and (3.27),

$$
\begin{aligned}
\left\|\underline{B}^{m}\right\|_{2} & =\left\|\left(I+h \underline{C}_{1}\right)^{-1}\left(\underline{E}_{2} \underline{E}_{1}\right)^{m}\left(\underline{I}+h \underline{C}_{1}\right)\right\|_{2} \\
& =\left\|\left(\underline{I}+h \underline{E}_{1} \underline{H}\right)^{-1} \underline{H}^{-1 / 2}\left(\underline{G}_{2} \underline{G}_{1}\right)^{m} \underline{H}^{1 / 2}\left(\underline{I}+h \underline{F}_{1} \underline{H}\right)\right\|_{2} \\
& \leq\left\|\left(I+h \underline{F}_{1} \underline{H}\right)^{-1}\right\|_{2}\left\|\underline{H}^{-1 / 2}\right\|_{2}\left\|\left(\underline{G}_{2} \underline{G}_{1}\right)^{m}\right\|_{2}\left\|\underline{H}^{1 / 2}\right\|_{2}\left(\underline{I}+h \underline{F}_{1} \underline{H}\right) \|_{2} \\
& \leq\left\|\left(\underline{I}+h \underline{F}_{1} \underline{H}\right)^{-1}\right\|_{2}\left\|\underline{H}^{-1 / 2}\right\|_{2}\left\|\underline{H}^{1 / 2}\right\|_{2}\left\|\left(\underline{I}+h \underline{F}_{1} \underline{H}\right)\right\|_{2} \\
& \leq K_{2}, \text { a constant }
\end{aligned}
$$


Because $\left\|\mathrm{B}^{\mathrm{m}}\right\|_{2}$ is bounded by a constant independent of $\mathrm{m}$, the system of equations is stable.

Q.E.D.

Theorem 3: (Strang Perturbation Theorem) 29

If the difference system $\bar{\psi}^{n+2}=\subseteq(h) \bar{\psi}^{n}$

is stable, and if $Q(h)$ is a bounded family of operators, then the difference system

$$
\bar{\psi}^{n+2}=[\subseteq(h)+h Q(h)] \bar{\psi}^{n}
$$

is stable.

\section{Proof:}

(The argument $(h)$ of $\underline{C}(h)$ and $\underline{Q}(h)$ is hereafter dropped to simplify notation.)

$$
\text { Since } \bar{\psi}^{n+2}=\underline{C} \bar{\psi}^{n} \text { is stable, it follows from }
$$

the definition of numerical stability (Appendix $B$ ) that

$$
\begin{aligned}
& \left\|\underline{C}^{n}\right\|<K_{1} \text {, a constant independent of } n \\
& \text { Since } Q(h) \text { is a bounded operator; } \\
& \|Q\|<K_{2} \text {, a constant }
\end{aligned}
$$

Furthermore, assume that there exists a constant $T$ such that

$$
\mathrm{nh}<\mathrm{T} \text {, where } \mathrm{T} \text { is a constant independent of } \mathrm{n}(3.28 \mathrm{c})
$$

Also, if $\left\|\underline{C}^{n}\right\|<1$, then let $K_{1}>1$. Hence, 


$$
K_{1}^{j} \geqslant K_{1} \quad j=1,2,3, \cdots, \infty
$$

Therefore,

$$
\begin{aligned}
\left\|(\underline{C}+h \underline{Q})^{n}\right\|= & \|(\underline{C}+h \underline{Q})(\underline{C}+h \underline{Q}) \cdots(\underline{C}+h \underline{Q})\| \\
= & \| \underline{C}^{n}+h \underline{Q} \underline{C}^{n-1}+h \underline{C} \underline{Q} \underline{C}^{n-2}+h \underline{C}^{2} \underline{Q} \underline{C}^{n-3}+\cdots+h \underline{C}^{n-1} \underline{Q} \\
& +h^{2} \underline{Q}^{2} \underline{C}^{n-2}+\cdots+h^{2} \underline{C}^{n-2} \underline{Q}^{2}+\cdots+h^{n} \underline{Q} \underline{Q}^{n} \| \\
\leq & \left\|\underline{C}^{n}\right\|+h\|\underline{Q}\| \underline{C^{n-1}}\|+h\| \underline{C}\|\underline{Q}\|\left\|\underline{C^{n-1}}\right\|+\cdots+h\left\|\underline{C}^{n-1}\right\|\|\underline{Q}\| \\
& +h^{2}\|\underline{Q}\| \underline{C}^{2}\left\|\underline{C^{n-2}}\right\|+\cdots+h^{2}\left\|\underline{C}^{n-2}\right\|\|\underline{Q}\|^{2}+\cdots+h^{n}\|\underline{Q}\| \\
< & K_{1}+h K_{1} K_{2}+h K_{1}^{2} K_{2}+\cdots+h K_{1} K_{2} \\
& +h^{2} K_{2}^{2} K_{1}+\cdots h^{2} K_{1} K_{2}^{2}+\cdots h^{n} K_{2}^{n}
\end{aligned}
$$

This finite series may be expressed as $\sum_{l=0}^{n}\left(\begin{array}{l}n \\ l\end{array}\right) K_{1}^{l+1}\left(h K_{2}\right)^{l}$ when use is made of Eq. (3.28d). Therefore,

$$
\begin{aligned}
& \left\|(\underline{C}+h \underline{Q})^{n}\right\|<K_{1} \sum_{l=0}^{n} \frac{n !}{(n-l) ! l !}\left(h K_{1} K_{2}\right)^{l} \\
& \text { Since } \\
& \quad n^{l} \geqslant \frac{n !}{(n-l) !}, \\
& K_{1} \sum_{l=0}^{n} \frac{n !}{(n-l) ! l !}\left(h K_{1} K_{2}\right)^{l} \leq K_{1} \sum_{l=0}^{n} \frac{\left(n h K_{1} K_{2}\right)^{l}}{l !}
\end{aligned}
$$


From Eq. (3.28c), $\quad n h<T$

$$
\begin{aligned}
& K_{1} \sum_{l=0}^{n} \frac{\left(n h K_{1} K_{2}\right)^{l}}{l !}<K_{1} \sum_{l=0}^{n} \frac{\left(K_{1} K_{2} T\right)^{l}}{l !} \\
& \text { Since } e^{K_{1} K_{2} T}=\sum_{l=0}^{\infty} \frac{\left(K_{1} K_{2} T\right)^{l}}{l !} \\
& K_{1} \sum_{l=0}^{n} \frac{\left(K_{1} K_{2} T\right)^{l}}{l !}<K_{1} e^{K_{1} K_{2} T}
\end{aligned}
$$

Therefore,

$$
\|(\underline{C}+h \underline{Q})^{n \|}<K_{1} e^{K_{1} K_{2} T}<K_{3}, \text { a constant }
$$

Because $\|\underline{C}(h)+h \underline{Q}(h)\|$ is bounded by a constant independent of $n$, the system of equations is stable. Q.E.D.

The stability of alternating direction methods may

be determined from the matrix properties of the principal part of the A-matrix. Because of the consistency restriction that the ratios

$$
r_{x}=\frac{h}{h_{x}^{2}} ; \quad r_{y}=\frac{h}{h_{y}^{2}}
$$

are fixed, 


$$
\lim _{h \rightarrow 0} h \underline{A}=\underline{D}
$$

and thus the D matrix of the splitting

$$
\underline{A}=\underline{D}+\underline{R}
$$

is the principal part of $\underline{A}$. (Actually the principal part does not include the diagonal terms of the precursor equation, but whether or not these terms are considered to be part of the matrix $\underline{D}$ does not alter the conclusions of the stability analysis.)

Let the principal part, $\underline{D}$, of the matrix $\underline{A}$ be divided as

$$
\underline{D}=-\underline{C}_{1}-\underline{C}_{2}
$$

where

$$
\begin{aligned}
& \underline{C}_{1}=-\underline{D}_{1} ; \underline{C}_{2}=-\underline{D}_{2} \text { for ADE methods } \\
& \underline{C}_{1}=-\underline{D}_{\mathrm{y}} ; \underline{C}_{2}=-\underline{D}_{\mathrm{x}} \text { for ADI methods } \\
& \underline{C}_{1}=-\underline{I}_{\mathrm{x}} \underline{D}_{2} \underline{\mathrm{C}}_{2}=-\underline{I}_{0} \underline{\mathrm{D}} \text { for checkerboard methods }
\end{aligned}
$$

Then the advancement matrix, $\underline{B}$, of the alternating direction method difference system,

$$
\bar{\psi}^{n+2}=\underline{B} \bar{\psi}^{n}
$$

may be factored as

$$
\underline{B}=\left\{\left[\underline{I}+h \underline{C}_{1}\right]^{-1}\left[\underline{I}-h \underline{C}_{2}\right]\left[\underline{I}+h \underline{C}_{2}\right]^{-1}\left[\underline{I}-h \underline{C}_{1}\right]+h \underline{Q}(h)\right\}
$$


where $\left(\underline{C}_{1}+\underline{C}_{2}\right)=\underline{C}$ is the principal part of $\underline{A}$ and where $\underline{Q}(h)$ is a bounded family operators. This is proven by Ferguson 21 for ADI and ADE methods with the single-level frequency transform advancement matrix,

$$
\begin{aligned}
B=e^{\Omega} h & {\left[I-h\left(\underline{A}_{4}-\alpha \underline{\Omega}\right)\right]^{-1}\left[I+h\left(\underline{A}_{3}-\gamma \underline{\Omega}\right)\right] } \\
\times & {\left[I-h\left(\underline{A}_{2}-\alpha \underline{\Omega}\right)\right]^{-1}\left[I+h\left(\underline{A}_{1}-\gamma \underline{\Omega}\right)\right] e^{\Omega h} }
\end{aligned}
$$

A similar proof is carried out in Appendix C for ADI, $\mathrm{ADE}$ and checkerboard methods with the two-level frequency transform advancement matrix,

$$
\begin{aligned}
\underline{B}= & {\left[I-h\left(\underline{A}_{4}-\alpha \Omega\right)\right]^{-1} e^{\Omega h}\left[I+h\left(\underline{A}_{3}-\gamma \underline{\Omega}\right)\right] } \\
& \times\left[I-h\left(\underline{A}_{2}-\alpha \Omega\right)\right]^{-1} e^{\Omega h}\left[I+h\left(\underline{A}_{1}-\gamma \Omega\right)\right]
\end{aligned}
$$

Therefore, by Theorem 3 the alternating direction method difference system

$$
\bar{\psi}^{n+2}=B \bar{\psi}^{n}
$$

is stable if the system

$$
\bar{\psi}^{n+2}=\left[\underline{I}+h \underline{C}_{1}\right]^{-1}\left[I-h \underline{C}_{2}\right]\left[\underline{I}+h \underline{C}_{2}\right]^{-1}\left[I-h \underline{C}_{1}\right] \bar{\psi}^{n}
$$

is stable. Hence, stability is determined from the matrix properties of the principal part of $\underline{A}$. 
The stability of the ADI and ADE methods follows from Theorem 2. The matrices $\left(\underline{D}_{1}+\underline{D}_{1}^{T}\right),\left(\underline{D}_{2}+\underline{D}_{2}^{T}\right),\left(\underline{D}_{x}+\underline{D}_{x}^{T}\right)$ and $\left(\underline{D}_{y}+\underline{D}_{y}^{T}\right)$ are all negative definite. Therefore, $\left(\underline{C}_{1}+\underline{C}_{1}^{T}\right)$ and $\left(\underline{C}_{2}+\underline{C}_{2}^{T}\right)$ are positive definite. In Theorem C.I (Appendix $C$ ) is is shown that the norm $\left\|\left(I+h \underline{C}_{1}\right)^{-1}\right\|_{2}$ is bounded and Theorem.C. 3 may be used to show that the norm $\left\|\left(I+h \underline{C}_{1}\right)\right\|_{2}$ is bounded. Hence, the first set of conditions in Theorem 2 is met and the ADI and ADE methods are numerically stable.

For checkerboard methods, $\left(\underline{C}_{1}+\underline{c}_{1}^{T}\right)$ and $\left(\underline{c}_{2}+\underline{c}_{2}^{T}\right)$ are not positive definite. However, $\underline{c}_{1}$ and $\underline{\mathrm{C}}_{2}$ may be factored as

$$
\underline{C}_{1}=-\underline{I}_{x} \underline{D}=\underline{F}_{1} \underline{H} \quad ; \quad \underline{C}_{2}=-\underline{I}_{0} \underline{D}=\underline{F}_{2} \underline{H}
$$

The matrices $\underline{F}_{1}$ and $\underline{F}_{2}$ are symmetric and positive definite:

$$
\underline{F}_{1}=\underline{I}_{x}=\underline{F}_{1}^{T / 2} \underline{F}_{1}^{I / 2} ; \underline{F}_{2}=\underline{I}_{0}=\underline{F}_{2}^{T / 2} \underline{F}_{2}^{l / 2}
$$

For N-dimensional problems, $\underline{H}=-\underline{D}$ is a $2 \mathrm{~N}+1$ stripe positive definite symmetric matrix. Therefore, $\underline{H}=\underline{H}^{T / 2} \underline{H}^{1 / 2}$ and the matrix $\underline{H}^{\mathrm{T} / 2}$ consists of a diagonal stripe and $\mathrm{N}$. upper diagonal stripes; the matrices $\underline{H}^{1 / 2}$ and $\underline{H}^{-1 / 2}$ consist of a diagonal stripe and $\mathrm{N}$ lower diagonal stripes. Because of this structure it can be shown by Theorem C.3 of Appendix $c$ that $\|(I+h E, H)\|_{2},\left\|\underline{H}^{1 / 2}\right\|_{2}$ and $\left\|\underline{H}^{-1 / 2}\right\|_{2}$ are bounded. Also, the boundedness of $\left\|\left(I+h E_{1} \underline{H}\right)^{-1}\right\|_{2}$ 
may be shown by Theorem C.2. Hence, the second set of conditions in Theorem 2 is met and the checkerboard methods are numerically stable.

The stability discussed in this section is known as unconditional stability in the sense that no restriction or condition has been placed on the constant ratios $r_{x}$ and $r_{y}$. This notion of stability should not be interpreted as an assurance of numerical accuracy. Indeed, errors may grow exponentially as is implied by Theorem 3 . Stability and consistency merely are indicators that as $h$ is decreased the solution of the difference equations will converge to that of the differential neutron diffusion equation.

It should also be noted that nothing has been said about the time dependence of $\underline{A}$. In the stability proof it is assumed that the principal part of $A$ is constant for all time, $t$. This is rarely true for reactor kinetics problems. However, numerical testing indicates that the time dependence of the A-matrix does not destroy convergence.

3.4 Numerical Results

The ADC method, the two-level frequency transform and the alternative checkerboard methods are numerically tested in this section. In addition, some variations of the ADC method are also examined. 
3.4a Numerical Assessment of the ADC Method and Several Variations of the ADC Method

There are many possible variations of the ADC method. One variation is to apply a two-level kinetics matrix technique; another variation is to change the pattern of solution of the ADC method; still another variation is to adjust artifically the group neutron velocities. These possibilities are now examined.

The alternating direction methods described so far use a single-level kinetics matrix technique. When the time dependence of the A-matrix is specified, Eqs. (1.9a) and (1.9b) may be written as

$$
\begin{aligned}
& \frac{\bar{\psi}^{n+1}-\bar{\psi}^{n}}{h}=\underline{A}_{1}\left(t^{k}\right) \bar{\psi}^{n}+\underline{A}_{2}\left(t^{k}\right) \bar{\psi}^{n+1} \\
& \frac{\bar{\psi}^{n+2}-\bar{\psi}^{n+1}}{h}=\underline{A}_{3}\left(t^{k}\right) \bar{\psi}^{n+1}+\underline{A}_{4}\left(t^{k}\right) \bar{\psi}^{n+2}
\end{aligned}
$$

This is a single-level kinetics matrix technique because the split matrices, $\underline{A}_{1}, \underline{A}_{2}, \underline{A}_{3}$ and $\underline{A}_{4}$ are all evaluated at the single time level, $t=t^{k}$. Alternatively, the matrices $\underline{A}_{1}, \underline{A}_{2}, \underline{A}_{3}$ and $\underline{A}_{4}$ may be evaluated at different levels in time as 


$$
\begin{aligned}
& \frac{\bar{\psi}^{n+1}-\bar{\psi}^{n}}{h}=\underline{A}_{1}\left(t^{n}\right) \bar{\psi}^{n}+\underline{A}_{2}\left(t^{n+1}\right) \bar{\psi}^{n+1} \\
& \frac{\bar{\psi}^{n+2}-\bar{\psi}^{n+1}}{h}=\underline{A}_{3}\left(t^{n+1}\right) \bar{\psi}^{n+1}+\underline{A}_{4}\left(t^{n+2}\right) \bar{\psi}^{n+2}
\end{aligned}
$$

This is a two-level kinetics matrix technique because the matrices in Eq. (3.30a) are evaluated at two different time levels and the matrices in Eq. (3.30b) are evaluated at two different time levels. Only minor modifications are required to use the two-level kinetics matrix technique in the ADC code, but it is rather difficult to program the NSADE and NSADI methods as two-level kinetics matrix technique methods.

The solution pattern of the ADC method is another variation which can be easily implemented in the ADC code. The ADC method described in section 3.2a uses the pattern XooX for each time step: in the first half-step the $x$-points are treated explicitly and then the o-points are treated implicitly; in the second half-step the o-points are treated explicitly and then the $x$-points are treated implicitly. The repeated pattern is

$$
/ \mathrm{xOOX} / \mathrm{xOOX} / \mathrm{xOOX} / \mathrm{xOOX} / \ldots
$$

An alternative pattern is 


$$
\text { / oxxo / oxxo / oxxo / oxxo / ... }
$$

The difference between these two patterns is simply that the $\mathrm{x}$ - and o-mesh points of the checkerboard mesh grid are interchanged.

Two other solution patterns are

$$
\text { / xoox / oxxo / xoox / oxxo / .. }
$$

and

$$
\text { / oxxo / xoox / oxxo / xoox / .. }
$$

These patterns are similar to each other but fundamentally different from the patterns of Eqs. (3.3la) and (3.31b). The stability analysis of Section $3.3 \mathrm{c}$ does not apply to the ADC method variation which uses the solution pattern of either Eq. (3.3Ic) or Eq. (3.3ld). Numerical testing indicates that checkerboard methods which use either of these two patterns are unacceptably inaccurate.

A final method variation is one in which the group neutron velocities, $\mathrm{v}_{\mathrm{g}}$, are artificially adjusted. The fully explicit treatment of some ADC mesh points is highly erroneous for problems in which the fast group neutron velocities are much larger than the thermal group neutron velocity. For these problems it is useful to adjust 
artifically the ADC fast group velocities.*

The ADC method and some of the above variations may be assessed by numerical testing.

In Table 3.3 the NSADE, NSADI, and ADC solutions of a negative step perturbation problem, Test Problem 3.1, are compared. Test Problem 3.1 is the same as Test Problem 2.4 in Chapter 2. The reactor modeled in this problem is described by Critical Configuration No. 3 in Appendix D. The two-dimensional NSADE, NSADI, and ADC codes treat this one-dimensional problem by setting the $y$-dimension mesh spacing equal to $10^{30} \mathrm{~cm}$. The transient of this problem is initiated by increasing the capture cross section by $1.36 \%$ in one half of the reactor. Tabulated fluxes are measured $1 / 4$ way across the reactor in the center of the perturbed half.

The ADC solutions tabulated in Table 3.3 are much more accurate than the NSADE and NSADI solutions. The NSADE and NSADI results are poor because the reactor buckling, $\nabla^{2} \phi(\bar{r}, E, t) / \phi(\bar{r}, E, t)$ in Test Problem 3.1 is large. For ADE methods, errors build up as the reactor mesh is swept from one corner to the other, and then are cancelled as the mesh is swept back the other way. (See Section 3.1d.) When $\nabla^{2} \phi(\bar{r}, E, t) / \phi(\bar{r}, E, t)$ is large,

* Note: artificially increasing the neutron velocities to infinity (as in the prompt jump approximation of $\mathrm{Ch}$. 2) is not an acceptable variation of the ADC method because of the fully explicit treatment of ADC fluxes for some mesh points. 
TABLE 3.3

Test Problem 3.I: Nonuniform Negative Step Perturbation Applied to the Homogeneous 1-Group Reactor of Critical Configuration No. 3 Time step size $=.001 \mathrm{sec}(h=.0005 \mathrm{sec})$

\begin{tabular}{|c|c|c|c|c|c|c|}
\hline \multicolumn{2}{|c|}{ Time $=$} & .01 & $.03^{\circ}$ & .06 & .1 & .3 \\
\hline \multicolumn{2}{|c|}{$\begin{array}{l}\text { True } \\
\text { Solution }\end{array}$} & .5023 & .4946 & .4941 & .4935 & .4906 \\
\hline NSADE & * & .6889 & .6758 & .6608 & .6437 & .5841 \\
\hline NSADE ${ }^{b}$ & 1 & .6611 & .529 .6 & .3954 & .5168 & .4657 \\
\hline NSADI & * & . 7099 & .7172 & .7346 & .7810 & 6.1241 \\
\hline NSADI & 1 & .7244 & 1.2396 & negative & fluxes & \\
\hline ADC & $*$ & .3344 & .5051 & .4935 & .4934 & .4906 \\
\hline ADC & 2 & .3879 & .5023 & .4936 & .4935 & .4906 \\
\hline$A D C^{C}$ & * & .4711 & .5111 & .4951 & .4935 & .4906 \\
\hline$A D C^{C}$ & 2 & .4872 & .5080 & .4950 & .4935 & .4906 \\
\hline & $\begin{array}{l}* \\
1 \\
2\end{array}$ & $\begin{array}{l}\text { no fr } \\
\text { singl } \\
\text { two-l }\end{array}$ & $\begin{array}{l}\text { ncy } t r \\
\text { vel fr } \\
\text { frequ }\end{array}$ & $\begin{array}{l}\text { rm used } \\
\text { cy transf } \\
\text { transform }\end{array}$ & $\begin{array}{l}\text { orm used } \\
\text { used }\end{array}$ & \\
\hline
\end{tabular}

a. The "True Solution" is the converged WIGLPJ code solution using the WIGL' method. (See Chapter 2) The same solution may also be generated by the NSADE; NSADI, and ADC codes if smaller time step sizes are used.

b. Same as MITKIN.

c. Two-level kinetics matrix used. (See Section 2.1f.) 
the errors become too large to cancel completely, and ADE methods are inaccurate. For ADI methods the reactor mesh grid is swept line by line rather than point by point. The data of Table 3.3 indicates that this sweeping also leads to errors and Test Problem 3.1 is treated inadequately by $A D I$ methods.

In checkerboard methods, the mesh is not swept in any direction; instead, all the $\mathrm{x}$-points are treated together and then all the o-points are treated together for the first half-step; for the second half-step the process is reversed. For problems where $\nabla^{2} \phi(\bar{r}, E, t) / \phi(\bar{r}, E, t)$ is large, checkerboard methods are usually better than ADE and ADI methods.

Contrary to the theta method results of Table 2.4 in Chapter 2, the data of Table 3.3 indicates that there is little advantage to applying the two-level kinetics matrix technique to the ADC method for this problem. But it will be shown later that the two-level kinetics matrix technique is a desirable variation of the ADC method for some other problems.

In Table 3.4 the ADC and NSADE solutions of a second test problem, Test Problem 3.2, are compared. Test Problem 3.2 is the same as test case number eight of Reed ${ }^{19}$. This is a nonsymmetric heterogeneous reactor problem with four energy groups and one precursor group. The physics and 
TABLE 3.4

Test Problem 3.2: Nonuniform Ramp Perturbation Applied to the Nonsymmetric Heterogeneous 4-Group Reactor of Critical Configuration No. 5 .

Time step size $=.001 \mathrm{sec}(h=.0005)$

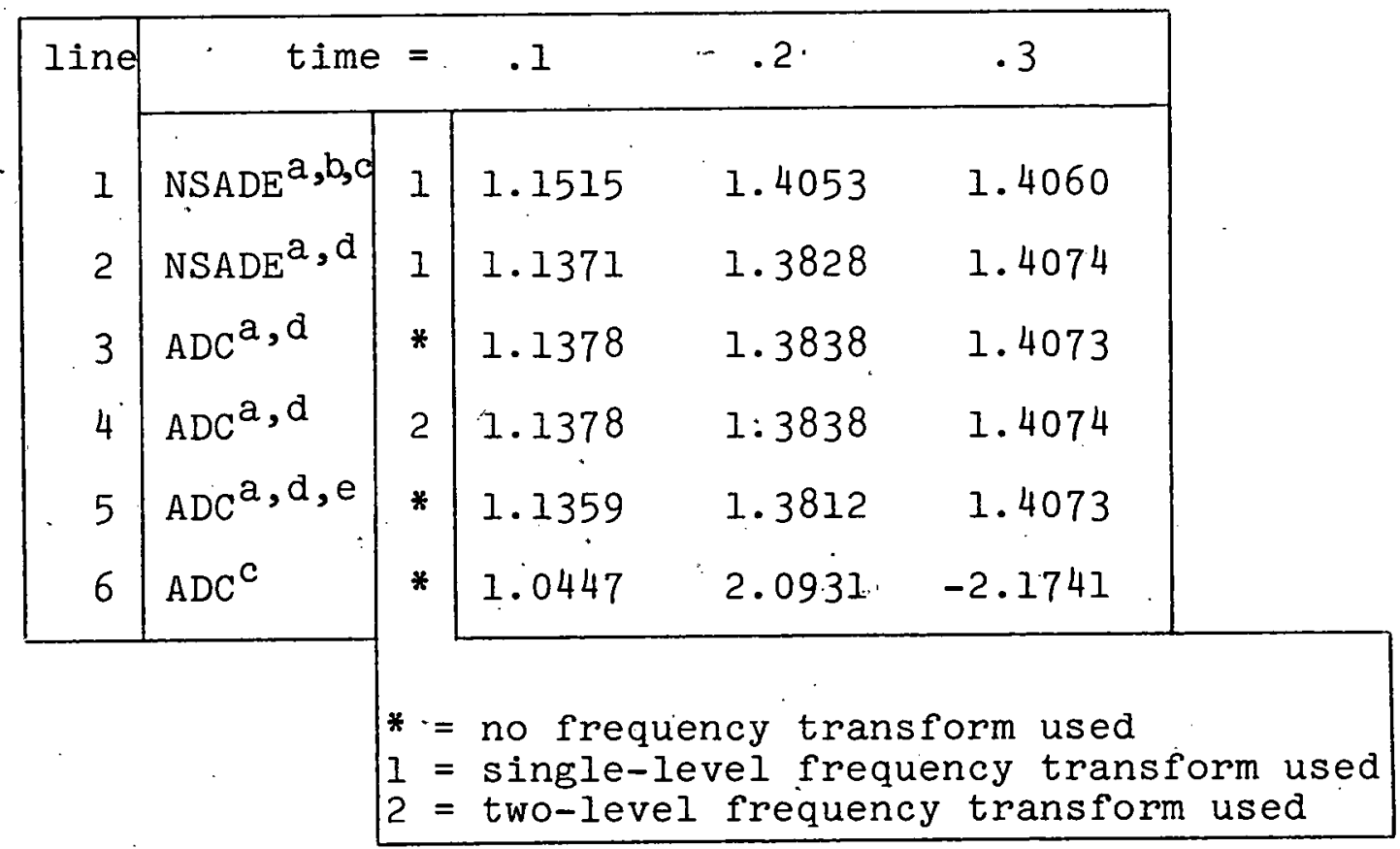

a. Solution converged to within . $2 \%$ accuracy.

b. Same as MITKIN.

c. True problem velocities used

d. All neutron velocities set equal to the thermal group neutron velocity.

e. $x$ - and o-mesh points order of solution changed. 
geometry of this problem are described by Critical

Configuration No. 5 in Appendix D. The transient analyzed is a positive ramp perturbation in which the macroscopic thermal cross section is decreased linearly in off-center reactor region \#(2,1). (See Appendix D.) The ramp perturbation of the thermal capture cross section is

$$
\begin{aligned}
& \Sigma_{c_{421}}(t)=\Sigma_{c_{421}}(0)-.015 t \quad \text { for } 0<t \leqslant .2 \mathrm{sec} \\
& \Sigma_{c_{421}}(t)=\Sigma_{c_{421}}(0)-.003=.009 \text { for } t>.2 \mathrm{sec}
\end{aligned}
$$

$$
\text { where } \begin{aligned}
\Sigma_{c_{421}}(t)= & \begin{array}{l}
\text { macroscopic thermal group }(G=4) \text { capture } \\
\text { cross section in spatial region } \#(2, I) \text { of } \\
\text { Critical Configuration No. } 5 \text { of Appendix } D .
\end{array} \\
\Sigma_{c_{421}}(0)= & .012 \mathrm{~cm}^{-1}=\text { initial critical value of } \\
& \Sigma_{c_{421}}(t) \text { for } t \leq 0 \text { sec. }
\end{aligned}
$$

The tabulated fluxes of this problem are the thermal fluxes (energy group $G=4$ ) measured at mesh point $(12,3$ ) which is located in the center of the perturbed region.

The fast group $(G=1)$ neutron velocity of Test Problem 3.2 is 5000 times larger than the thermal ( $G=4)$ group neutron velocity. This poses no difficulty for the NSADE code as shown in line 1 of Table 3.4. But when the ADC code is used, roundoff errors in the fast group are magnified by a factor of 5000 in the thermal group. (See Section 3.2d.) As shown by line 6 , the resulting ADC 
code errors become intolerably large and negative fluxes are computed for this problem*. For problems such as this, it is necessary to adjust artificially the fast group neutron velocities in the ADC code.

When all group neutron velocities, $\mathrm{v}_{\mathrm{g}}$, are set equal to the thermal group neutron velocity, $v_{G}$, the ADC method no longer exhibits excessive round off error problems. However, the reactor is now inaccurately modeled. Fortunately, the error introduced by approximating the multigroup neutron velocities as

$$
\mathrm{v}_{\mathrm{g}}=\mathrm{v}_{\mathrm{G}} \quad \mathrm{g}=1,2, \ldots, \mathrm{G}
$$

is small. Comparison of lines 1 and 2 of Table 3.4 indicates that the problem. solution is only changed by about $1 \%$ for this transient when the $v_{g}=v_{G}$ approximation is used. Hence, the ADC method is competitive with the NSADE method even for this problem in which the $v_{g}=v_{G}$ approximation must be used.

* The computation of negative fluxes does not contradict the stability of the ADC method. Let the true solution of Test Problem 3.2 at each time step be denoted by $\Phi_{1}^{n}$ and let the solution of line 6 be denoted by $\phi_{6}^{n}$. Then the absolute error at each time step, $n$, is

$$
\epsilon_{n}=\left|\phi_{1}^{n}-\phi_{6}^{n}\right|
$$

Numerical testing indicates that the growth of $\epsilon_{n}$ is bounded as

$$
\epsilon_{n}<c_{1} \exp \left(c_{2} n\right)
$$

where $c_{1}$ and $c_{2}$ are constants and the true solution, $\phi_{1}^{n}$, is assumed to be the solution of line 1 . This exponential error growth may be inferred from Theorem 3 of section 3:3c. 
In lines 3,4 , and 6 of Table 3.4 the ADC solution pattern is

$$
\text { / oxxo / oxxo / oxxo / oxxo / . . }
$$

but in line 5 the solution pattern is

$$
/ \operatorname{xoOx} / \mathrm{xOOx} / \mathrm{xOOx} / \mathrm{xOOX} / \ldots
$$

The methods of lines 3 and 5 in Table 3.4 differ only by the solution pattern order. The similarity of these solutions indicates that it makes little difference whether the $x$-mesh points are treated first or the o-mesh points are treated first by checkerboard methods. Alternatively stated, the $\mathrm{x}$ - and o-mesh points of the checkerboard mesh grid (Figure 3.2) may be interchanged without significantly affecting the accuracy of the checkerboard method.

The results of this section are consistent with some general observations:

1) The usefulness of the two-level kinetics matrix variation of the $A D C$ method is problem dependent;

2) The $x$ - and o-mesh points of the checkerboard mesh grid may be interchanged without significantly affecting the checkerboard method accuracy;

3) The ADC method is competitive with the NSADE method even for problems in which the neutron velocities must be artificially adjusted to reduce round off errors; 
4) The ADC method is better than other alternating direction methods for some problems.

$3.4 \mathrm{~b}$ Comparison of Frequency Transforms

In Table 3.5, Test Problem 3.3 is solved by four alternating direction methods. Test Problem 3.3 is the same as test case number one of Reed ${ }^{19}$. In this problem a two energy group, one precursor group, homogeneous reactor is modeled. The physics and geometry of this reactor are described by Critical Configuration No. 4 in Appendix D. The transient analyzed is a positive step perturbation in which the macroscopic capture cross section is abruptly decreased by $3.69 \times 10^{-5} \mathrm{~cm}^{-1}$ at time $t=0$ seconds. This corresponds to a prompt supercritical transient with about a $\$ 5$ reactivity insertion. The true solution of this problem for time $t=.4$ seconds has been determined analytically by Reed ${ }^{19}$ : the centerpoint thermal ( $G=2$ ) flux is $\varnothing=1.303$. Various time step sizes are used in Table 3.5 to calculate the tabulated centerpoint thermal fluxes at time $t=.4$ seconds.

Table 3.5 may be used to assess the single-level and two-level frequency transforms. The single-level frequency transform is not applied to the ADC and NSADC (see Section 3.2e) methods because it is incompatible* with checker-

* When the single-level frequency transform is applied to checkerboard methods, time step sizes several orders of magnitude smaller than those listed in Table 3.5 must be used in order to achieve acceptable accuracy. 
TABLE 3.5

Test Problem 3.3: Supercritical step Perturbation Applied to 2-Group Homogeneous Reactor of Critical Configuration No. 4

Centerpoint thermal fluxes tabulated at time $t=.4$ sec.

True Solution $=1.303$

\begin{tabular}{|c|c|c|c|c|c|}
\hline \multicolumn{2}{|c|}{$\begin{array}{c}\text { Time step } \\
\text { size (sec) } \\
\Delta=2 h=\end{array}$} & \multirow{2}{*}{$\frac{0.0008}{1.3028}$} & \multirow{2}{*}{$\frac{0.002}{1.3024}$} & \multirow{2}{*}{$\frac{0.004}{1.3006}$} & \multirow{2}{*}{$\frac{0.008}{1.2876}$} \\
\hline$A D C$ & $*$ & & & & \\
\hline ADC & 2 & & 1.3094 & 1.3300 & 1.4314 \\
\hline NSADE & * & 1.0291 & 0.8334 & 0.6840 & 0.5644 \\
\hline $\mathrm{NSADE}^{\mathrm{a}}$ & 1 & 1.3037 & 1.3070 & 1.3187 & 1.3718 \\
\hline NSADE & 2 & 1.3043 & 1.3087 & 1.3223 & 1.3804 \\
\hline NSADC & * & & 1.0787 & 0.9096 & 0.7291 \\
\hline NSADC & 2 & & 1.3035 & 1.3074 & 1.3264 \\
\hline SCADE & * & & 0.4588 & 0.4105 & 0.3972 \\
\hline SCADE & 1 & & 1.4098 & 1.2349 & 0.7992 \\
\hline SCADE & 2 & & 1.3671 & 1.2192 & 0.9026 \\
\hline & $\begin{array}{l}* \\
1 \\
2\end{array}$ & $\begin{array}{l}\text { no frequ } \\
\text { single- } \\
\text { two-lev }\end{array}$ & $\begin{array}{l}\text { transf } \\
\text { freque } \\
\text { requency }\end{array}$ & $\begin{array}{l}m \text { used } \\
\text { y trans } \\
\text { ransfor }\end{array}$ & $\begin{array}{l}\text { us ed } \\
\text { ed }\end{array}$ \\
\hline
\end{tabular}

a. Same as MITKIN. 
board methods (see Section 3.2b). Both frequency transforms are applicable to the NSADE and SCADE (see section 3.2e) methods. For Test Problem 3.3 the NSADE method is most accurate with the single-level frequency transform and the SCADE method is most accurate with the two-level frequency transform, but this is not always the case (see Table 3.6). In general, the single-level and two-level frequency transforms have similar accuracy for ADE methods but only the two-level frequency transform works well for checkerboard methods.

The use of a frequency transform increases the accuracy of all the methods listed in Table 3.5 except the accuracy of the ADC method. Why the use of a frequency transform will generally improve the accuracy of alternating direction methods exclusive of the ADC method is not readily apparent from the truncation error analyses of sections 3.Id and 3.2c. However, a conjecture explaining this phenomenon is possible.

It may be conjectured that methods which are in some sense either fully implicit or fully explicit are not greatly improved by the application of a frequency transform. This conjecture is consistent with the results of Tables 3.3-3.6. The ADC method, which treats each mesh point either fully implicitly or fully explicitly, is generally not improved when a frequency transform is 
applied. This conjecture is also consistent with the observations of others; 30 theta methods, which may be thought of as an averaging of fully implicit and explicit techniques, are not greatly improved by the application of a frequency transform. It has been found that the accuracy of fully implicit methods is only modestly increased by use of a frequency transform. Only splitting techniques which are in no sense fully implicit or explicit seem to be dramatically and consistently improved by the application of a frequency transform.

\section{4c Comparison of Alternative Methods}

In Section $3.2 \mathrm{e}$ three new methods alternative to the ADC method were described.

The first of these methods is described by Eqs. (3.24). This method is highly inaccurate unless unreasonably small time step sizes are used.

A better method is the symmetric SCADE method of. Section $3.2 \mathrm{e}$. However, this method is less accurate than the ADC method for most problems. (See Table 3.5.)

A final alternative method is the NSADC method. As shown in Table 3.5, the NSADC method with the two-level frequency transform is competitive with the ADC method and is more accurate than the NSADE (MITKIN) method. The NSADC method is the best of the methods described in Section $3.2 \mathrm{e}$ and is generally at least as good as the ADC and NSADE methods. 


\section{4d A Final Example}

In Table 3.6 a final set of data is presented in order to assess the $A D C$ method, the two-level frequency transform, and the best of the alternative checkerboard methods described in section $3.2 e$. The reactor problem of Table 3.6 is Test Problem 3.2; the time step size used is ten times larger than that which was used in Table 3.4 .

Test Problem 3.2 is examined because it is a benchmark problem of the MIT Department of Nuclear Engineering. It is a mathematically interesting problem, but it is also physically unrealistic. Initial condition flux values at neighboring mesh points vary by as much as an order of magnitude in some reactor regions. (See Critical Configuration No. 5 of Appendix D.) A consequence of these variations is large round off errors.

The ADC method is especially sensitive to round off errors because at each half-step either the $x$-mesh points or the o-mesh points are treated fully explicitly. These round off errors are partially reduced by setting all group neutron velocities equal to the thermal group neutron velocity. A further reduction of round off errors is made possible by use of the two-level kinetics matrix technique.

It may be argued that the two-level kinetics matrix splitting reduces ADC round off errors by avoiding the 
Test Problem 3.2: Nonuniform Ramp

Perturbation Applied to Nonsymmetric

Heterogeneous 4-Group Reactor of

Critical Configuration No. 5.

All neutron velocities are set equal

to the thermal group $(G=4)$ neutron

velocity.

Time step size $=.01 \mathrm{sec}(h=.005)$

\begin{tabular}{|c|c|c|c|c|c|c|}
\hline \multicolumn{2}{|l|}{ Time } & $=.1$ & .2 & .3 & .4 & .5 \\
\hline \multicolumn{2}{|c|}{$\begin{array}{l}\text { Converged } \\
\text { solutiona }\end{array}$} & 1.0371 & 1.3828 & 1.4074 & 1.4090 & 1.4103 \\
\hline NSADE & $*$ & 1.0961 & 1.2683 & 1.2877 & 1.3018 & 1.3142 \\
\hline$N S A D E^{b}$ & 1 & 1.1167 & 1.3692 & 1.4639 & 1.3754 & 1.2551 \\
\hline NSADE & 2 & 1.1080 & 1.3459 & 1.4557 & 1.4886 & 1.4360 \\
\hline ADC & $*$ & 1.2011 & 1.4791 & 1.3591 & 1.6856 & 1.4534 \\
\hline$A D C^{C}$ & $*$ & 1.1069 & 1.3440 & 1.4542 & 1.4579 & 1.4193 \\
\hline$A D C^{C}$ & 2 & 1.1070 & 1.3460 & 1.4609 & 1.4618 & 1.4189 \\
\hline NSADC & $*$ & 1.0973 & 1.2716 & 1.2909 & 1.3075 & 1.3216 \\
\hline NSADC & 2 & 1.1479 & 1.4545 & 1.5138 & 1.4709 & 1.4034 \\
\hline & $\begin{array}{l}* \\
1 \\
2\end{array}$ & $\begin{array}{l}=\text { no fre } \\
=\text { single } \\
=\text { two-le }\end{array}$ & $\begin{array}{l}\text { ncy tra } \\
\text { vel fre } \\
\text { freque }\end{array}$ & $\begin{array}{l}\text { form } \\
\text { ency tr } \\
y \text { trans }\end{array}$ & $\begin{array}{l}\text { orm use } \\
\text { used }\end{array}$ & \\
\hline
\end{tabular}

a. Converged solution is determined by MITKIN method with $\mathrm{h}=0.0005$.

b. MITKIN method.

c. Two-level kinetics matrix used. 
fully explicit ADC calculations. The two-level ADC equation for the first half-step is similar to Eq. (3.30a):

$$
\frac{\Psi^{n+1}-\bar{\Psi}^{n}}{h}=\underline{I}_{x} \underline{A}\left(t^{n}\right) \Psi^{n}+\underline{I}_{0} \underline{A}\left(t^{n+1}\right) \bar{\Psi}^{n+1}
$$

The equation for the $x$-mesh points is fully explicit:

$$
\frac{\bar{\psi}_{x}^{n+1}-\bar{\psi}_{x}^{n}}{h}=\underline{A}\left(t^{n}\right) \bar{\psi}_{x}^{n}
$$

Note that the equation for the $x$-mesh points in the previous half-step $\left(t^{n-1}<t \leqslant t^{n}\right)$ is fully implicit:

$$
\frac{\bar{\psi}_{x}^{n}-\bar{\psi}_{x}^{n-1}}{h}=A\left(t^{n}\right) \bar{\psi}_{x}^{n}
$$

When the two-level kinetics matrix technique is used the matrices in Eqs. (3.32a) and (3.32b) are identical.* Therefore, Eqs. (3.32a) and (3.32b) may be combined as

$$
\bar{\psi}_{x}^{n+1}=2 \bar{\psi}_{x}^{n}-\bar{\psi}_{x}^{n-1}
$$

* When the single-level kinetics matrix technique is used the matrix in Eq. (3.32b) is $A(t m)$ for which $t^{n-2}<t^{m} \leq t^{n}$ and the matrix in Eq. ( $3.32 \mathrm{a})$ is $A\left(t^{k}\right)$ for which $t^{n}<t^{k} \leqslant t^{n+2}$. 
This form is insensitive to round off errors. Because the two-level ADC method is equivalent* to a solution technique relatively insensitive to round off errors, it is conjectured that the two-level ADC method is less sensitive to round off errors than is the partly explicit single-level ADC method.

This conjecture is supported by the data of Table 3.6 . The (single-level) ADC solution is the least accurate solution tabulated, but the two-level ADC solution is the most accurate solution tabulated. In general, the two-level kinetics matrix technique increases ADC accuracy only for problems in which large round off errors are troublesome. For Test Problems 3.1 and 3.3, large round off errors do not limit the ADC method and hence the two-level kinetics matrix technique does not improve the ADC solution accuracy of these problems.

The data of Table 3.6 is consistent with observations which were mentioned earlier:

1) The two-level kinetics matrix variation of the ADC

* The two-level. ADC method may be programmed in two ways: explicit calculations may be programmed as in Eq. (3.32a) or by the equivalent form, Eq. (3.32c). The two-level ADC method using Eq. (3.32c) requires half as much computing time but twice as much computer storage as the two-level ADC method which uses Eq. (3.32a). The method of Eq. ( $3.32 a$ ) was used to generate the data of Table 3.6 because this method requires only trivial modification of the (single-level) ADC code. Presumably, the method of Eq. $(3.32 c)$ would give identical results. 
method is useful for some problems;

2) The single-level and two-level frequency transforms have similar accuracy for ADE methods, but only the two-level frequency transform works well for checkerboard methods;

3) The accuracy of the ADC method is not greatly improved by the use of a frequency transform as is the accuracy of other alternating direction methods;

4) Two checkerboard methods, the NSADC and the ADC methods, are competitive with the NSADE method.

3.5 Conclusions

Alternating direction methods may be divided into three categories differentiated by the treatment of diffusion terms: implicit, explicit, and checkerboard methods. The best of the implicit and explicit methods seems to be the NSADE method. The best checkerboard methods seem to be the ADC and NSADC methods. In the NSADE method the reactor mesh grid is swept from one corner to the other which may lead to poor accuracy in some reactor problems because errors are propagated across the mesh grid. (See Table 3.3.) In checkerboard methods this source of error is avoided, but perturbations are not propagated; this may also lead to inaccuracies in some reactor problems. (See Section 3.2d.)

The accuracy of most alternating direction methods is dramatically improved by the use of an exponential 
transformation. This transformation may take several forms, two of which are quite successful: the single-

level frequency transform of Reed 19 and the two-level frequency transform of this thesis. Both of these transforms are of comparable value when applied to the NSADE method, but only the two-level frequency transform is of value to checkerboard methods.

The ADC method does not require the use of a frequency transform in order to achieve good accuracy for large time step sizes. Therefore, the additional programming effort, program execution time, and computer storage requirements associated with the exponential transformation may be avoided. However, the ADC method is sensitive to roundoff errors and.compensating adjustments (such as artificially reducing the fast group neutron velocities) must be made for some problems. Also, a time consuming GxG full matrix inversion is required for each mesh point at each time step when the ADC method is used. The NSADC and NSADE methods do not have these disadvantages, but then a frequency transform is required by these methods in order to achieve good accuracy for large time step sizes.

In section 3.4 it was shown that checkerboard methods are better than $A D E$ and $A D I$ methods for some problems. For other problems the NSADE, NSADC, and ADC methods are competitive. Apparently the NSADC and ADC checkerboard 
methods are at least as good as the NSADE and other alternating direction methods. 


\section{REFERENCES}

1. G. Strang and G.J. Fix, An Analysis of the Finite

Element Method, Prentice-Hall, Inc., Englewood Cliffs, N.J. (I973).

2. C.M. Kang and K.F. Hansen, "Finite Element Methods for Reactor Analysis," Nucl. Sci. Eng., 51, 456 (1973).

3. C.G. Chezem and W.H. Kohler, Eds., "Coupled Reactor Kinetics," Proceedings of the National Topical Meeting of the American Nuclear Society, Texas, January 1967.

4. J.M. Sicilian, "Response Matrices in Space-Time Reactor Dynamics," Nucl. Sci. Eng., 56, 291 (1975).

5. S. Kaplan, "The Property of Finality and the Analysis of Problems in Reactor Space-Time Kinetics by Various Modal Expansions," Nucl. Sci. Eng. 9, 357 (1961).

6. S. Kaplan, O.J. Marlowe, and J. Bewick, "Application of Synthesis Techniques to Problems Involving Time Dependence," Nucl. Sci. Eng. 18, 163 (1964).

7. A.F. Henry, "Nuclear Reactor Physics III," 22.213 Course Notes, Massachusetts Institute of Technology (1974), unpublished.

8. K. Ott, "Quasistatic Treatment of Spatial Phenomena in Reactor Dynamics," Nucl. Sci. Eng., 26, 563 (1966).

9. M. Clark, Jr., and K.F. Hansen, Numerical Methods of Reactor Analysis, Academic Press, New York (1964).

10. W.R. Cadwell, A.F. Henry, and A.J. Vigilotti, "WIGLEA Program for the Solution of the Two-Group, SpaceTime Diffusion Equations in Slab Geometry," WAPD-TM-416, Bettis Atomic Power Laboratory (1964).

11. J.B. Yasinsky, M. Natelson, and L.A. Hageman, "TWIGLA Program to Solve the Two-Dimensional, Two-Group, Space-Time Neutron Diffusion Equations with Temperature Feedback," WAPD-TM-743, Bettis Atomic Power Laboratory (1968).

12. W.H. Reed and K.F. Hansen, "Alternating Direction Methods for Reactor Kinetics Equations," Nucl. Sci. Eng., 4I, 431 (1970). 
13. D.L. Hetrick, Dynamics of Nuclear Reactors, University of Chicago Press (1971).

14. S. Glasstone and A. Sesonske, Nuclear Reactor Engineering, Van Nostrand Reinhold Co., New. York (1955).

15. A.F. Henry, "Nuclear Reactor Kinetics,". 22.22 Course Notes, Massachusetts. Institute of Technology (1974), unpublished.

16. F.B. Hildebrand, Methods of Applied Mathematics, Second Edition, Prentice-Hall, Inc., Englewood Cliffs, N.J. (1965).

17. W. Werner, "Mathematical Methods Applicable to Space Time Kinetics," Proc. of a Specialist Meeting on "Reactivity Effects in Large Power Reactors" at Ispra, Italy, October 28-31, 19.70. Commission of the European Communities, EUR $4731 \mathrm{f}-\mathrm{e}$, Luxembourg (1972).

18. D.W. Peaceman and H.H. Rachford, Jr., "The Numerical Solution of Parabolic and Differential Equations," J. Soe. Ind. Appl. Math., $\underline{3}, 28$ (1955).

19. W.H. Reed, "Finite Difference Techniques for the Solution of the Reactor Kinetics Equations," Sc.D. Thesis, Department of Nuclear Engineering, Massachusetts Institute of Technology, MITNE-100 (1969).

20. A.L. Wight, K.F. Hansen, and D.R. Ferguson, "Application of Alternating-Direction Implicit Methods to Space-Dependent Kinetics Equations," Nucl. Sci. Eng., 44, 239 (1971).

21. D.R. Ferguson and K.F. Hansen, "Solution of the SpaceDependent Reactor Kinetics Equations in Three Dimensions," Sc.D. Thesis, Department of Nuclear Engineering, Massachusetts Institute of Technology, MITNE-132 (1971).

22. L.A. Hageman and J.B. Yasinsky, "Comparison of Alternating-Direction Time-Differencing Methods With Other Implicit Methods for the Solution of the Neutron Group-Diffusion Equations," Nucl. Sci. Eng., 38, 8 (1969).

23. A.L. Wight, "The Application of Alternating-Direction Implicit Methods to the Space-Dependent Kinetics Equations," Ph.D. Thesis, Department of Nuclear Engineering, Massachusetts Institute of Technology, MITNE-106 (1969). 
24. K.F. Hansen and A.F. Henry, Progress Report No. 26, AEC Research Contract AT(11-1)-2262 (1974). (unpublished).

25. A.R. Gourlay, "Hopscotch: A Fast Second-Order Partial Differential Equation Solver," J. Inst. Maths. Applics., 6, 375 (1970).

26. F.B. Hildebrand, Introduction to Numerical Analysis, 2nd Edition, McGraw-Hill, New York (1974).

27. R.D. Richtmyer and K.W. Morton, Difference Methods for Initial-Value Problems, John Wiley and Sons; New York (1967).

28. R.B. Kellogg, "An Alternating Direction Method for Operator Equations," J. Soc. Ind. Appl. Math., 12, 848 (1964).

29. K.F. Hansen, 22.43 Class Notes, Massachusetts Institute of Technology (1974), unpublished.

30. J.W. Stewart, Savannah River Laboratory, personal communication.

31. R.S. Varga, Matrix Iterative Analysis, Prentice-Hall, Inc., Englewood Cliffs, New Jersey (1962). 
Appendix A

RELEVANT EQUATIONS

\section{A.1 The Neutron Diffusion Equation}

The time, space, and energy dependent neutron diffusion equation is ${ }^{15}$ :

$$
\begin{aligned}
\frac{1}{v(E)} \frac{\partial \phi(\bar{r}, E, t)}{\partial t} & =\bar{\nabla} \cdot D(\bar{r}, E, t) \nabla \phi(\bar{r}, E, t)-\Sigma_{t}(\bar{r}, E, t) \phi(\bar{r}, E, t) \\
& +\int_{0}^{\infty}\left[(1-\beta) x(E) i \Sigma_{f}\left(\bar{r}, E^{\prime}, t\right)\right. \\
& \left.+\Sigma_{s}\left(\bar{r}, E^{\prime} \rightarrow E, t\right)\right] \phi\left(\bar{r}, E^{\prime}, t\right) d E^{\prime} \\
& +\sum_{i=1}^{I} f_{i}(E) \lambda_{i} C_{i}(\bar{r}, t)+q(\bar{r}, E, t)
\end{aligned}
$$

where the delayed neutron precursor concentrations are found by

$$
\begin{aligned}
& \frac{\partial C_{i}(\bar{r}, t)}{\partial t}=\int_{0}^{\infty} B_{i} \nu \Sigma_{f}\left(\bar{r}, E^{\prime}, t\right) \phi\left(\bar{r}, E^{\prime}, t\right) d E^{\prime}-\lambda_{i} C_{i}(\bar{r}, t) \\
& i=1,2, \cdots, I \\
& i=\text { index of precursor groups, } i=1,2, \ldots, I \\
& \bar{r}=\begin{array}{c}
\text { spatial vector describing neutron location } \\
(\mathrm{cm})
\end{array}
\end{aligned}
$$




$$
\begin{aligned}
& E \text { = energy (ev) } \\
& \mathrm{t}=\mathrm{time} \\
& \phi(\bar{r}, E, t)=\text { neutron scaler flux (neutrons } / \mathrm{cm}^{2} / \mathrm{sec} \text { ) } \\
& C_{i}(\bar{r}, t)=i \text { th precursor concentration (atoms } / \mathrm{cm}^{3} \text { ) } \\
& \mathrm{v}(\mathrm{E})=\text { neutron velocity }(\mathrm{cm} / \mathrm{sec}) \\
& D(\bar{r}, E, t)=\text { diffusion coefficient }(\mathrm{cm}) \\
& \lambda_{i}=\text { decay constant of ith precursor group } \\
& \left(\sec ^{-1}\right) \\
& \Sigma_{t}(\bar{r}, E, t)=\text { total interaction cross section }\left(\mathrm{cm}^{-1}\right) \\
& X(E)=\text { prompt neutron spectrum } \\
& \nu=\text { average number of neutrons produced per } \\
& \text { fission } \\
& \begin{aligned}
\Sigma_{f}(\bar{r}, E, t)= & \text { fission cross section }\left(\mathrm{cm}^{-} 1\right) ; \text { this also } \\
& \text { includes }(n, 2 n),(n, 3 n), \text { etc. }
\end{aligned} \\
& B_{i}=\underset{\text { delayed neutron fraction of }}{\text { group precursor }} \\
& \beta=\sum_{i=1}^{I} \beta_{i} \\
& \Sigma_{S}\left(\bar{r}, E^{\prime} \rightarrow E, t\right)=\begin{array}{l}
\text { differential scattering cross section from } \\
\text { energy } E^{\prime} \text { to energy } E\left(\mathrm{~cm}^{-1}\right)
\end{array} \\
& f_{i}(E)=\text { delayed neutron spectrum }
\end{aligned}
$$

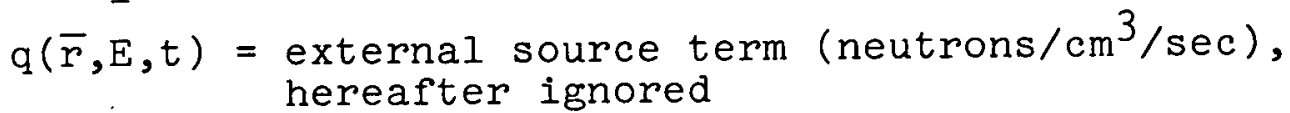

\section{A.2 The Point Kinetics Equations}

In order to derive the point kinetics equations the following change of variables is useful:

$$
\phi(\bar{r}, E, t) \equiv \phi(t) S(\bar{r}, E, t)
$$


$\phi(t)$ is the point kinetics (space and energy depen-. dent) flux. The shape function, $S(\bar{r}, E, t)$ is normalized by an energy and volume integral:

$$
\int_{V} \int_{E} \frac{1}{V(E)} S(\bar{r}, E, t) d E d V \equiv 1
$$

The next step of the point kinetics derivation is to integrate Eq. (A.Ia) over volume and energy and to integrate Eq. (A.lb) over volume, using the above change of variables in both cases.

$$
\begin{aligned}
& \iint_{V} \frac{1}{V(E)} S(\bar{r}, E, t) d E d V \frac{\partial \phi(t)}{\partial t} \\
& \quad \int_{V} \int_{E}\left[\bar{\nabla} \cdot D(\bar{r}, E, t) \nabla S(\bar{r}, E, t)-\Sigma_{t}(\bar{r}, E, t) S(\bar{r}, E, t)\right] d E d V \phi(t) \\
& +\int_{V} \int_{E} \int_{E^{\prime}}\left[(1-\beta) X(E) \nu \Sigma_{f}\left(\bar{r}, E^{\prime}, t\right)+\Sigma_{S}\left(\bar{r}, E^{\prime} \rightarrow E, t\right)\right] S\left(\bar{r}, E^{\prime}, t\right) d E^{\prime} d E d V \phi(t) \\
& \quad+\int_{V} \int_{E} \sum_{i=1}^{I} f_{i}(E) \lambda_{i} C_{i}(\bar{r}, t) d E d V \\
& \frac{\partial}{\partial t} \int_{V} C_{i}(\bar{r}, t) d V=\int_{V} \int_{E^{\prime}} \beta_{i} \nu \Sigma_{f}\left(\bar{r}, E^{\prime}, t\right) S\left(\bar{r}, E^{\prime}, t\right) d E^{\prime} d V \phi(t) \\
& \quad-\lambda_{i} \int_{V} C_{i}(\bar{r}, t) d V \quad \text { (A. 4a) }
\end{aligned}
$$


Note that

$$
\begin{aligned}
& \int_{E} X(E) d E \equiv 1 \\
& \int_{E} f_{i}(E) d E \equiv 1 \quad \text { (A.5a) }
\end{aligned}
$$

and

$$
\int_{E} \Sigma_{S}\left(\bar{r}, E^{\prime} \rightarrow E, t\right) d E=\Sigma_{t}\left(\bar{r}, E^{\prime}, t\right)-\Sigma_{a}\left(\bar{r}, E^{\prime}, t\right)
$$

where $\Sigma_{a}\left(\bar{r}, E^{\prime}, t\right)$ is the absorption cross section. Eqs. (A.5) may be used in Eq. (A.4a) to give

$$
\begin{aligned}
\frac{\partial \phi(t)=}{\partial t} \int_{V} \int_{E}\left[\bar{\nabla} \cdot D(\bar{r}, E, t) \nabla S(\bar{r}, E, t)-\Sigma_{a}(\bar{r}, E, t) S(\bar{r}, E, t)\right. \\
\left.+(1-\beta) \nu \Sigma_{f}(\bar{r}, E, t) S(\bar{r}, E, t)\right] d E d V \phi(t) \\
+\sum_{i=1}^{I} \lambda_{i} \int_{V} C_{i}(\bar{r}, t) d V
\end{aligned}
$$

Now the point kinetics parameters are defined:

$\rho(t) \equiv \frac{\int_{V} \int_{E}\left[\bar{\nabla} \cdot D(\bar{r}, E, t) \nabla S(\bar{r}, E, t)-\Sigma_{a}(\bar{r}, E, t) S(\bar{r}, E, t)+\nu \Sigma_{f}(\bar{r}, E, t) S(\bar{r}, E, t)\right] d E d V}{\int_{V} \int_{E} \nu \Sigma_{f}(\bar{r}, E, t) S(\bar{r}, E, t) d E d V}$ 


$$
\Lambda(t) \equiv \frac{1}{\int_{V} \int_{E} \nu \Sigma_{f}(\bar{r}, E, t) S(\bar{r}, E, t) d E d V}
$$

$$
C_{i}(t) \equiv \int_{\nabla} C_{i}(\bar{r}, t) d V
$$

$$
i=1,2, \cdots, I
$$

Inserting these definitions into Eqs. (A.6) and (A.4b) leads to the point kinetics equations:

$$
\begin{aligned}
& \frac{\partial \phi(t)}{\partial t}=\frac{\rho(t)-\beta}{\Lambda(t)} \phi(t)+\sum_{i=1}^{I} \lambda_{i} C_{i}(t) \\
& \frac{\partial C_{i}(t)}{\partial t}=\frac{\beta_{i}}{\Lambda(t)} \phi(t)-\lambda_{i} C_{i}(t) \quad \\
& i=1,2, \cdots, I
\end{aligned}
$$

Note that as $\frac{1}{V(E)} \rightarrow 0, S(\bar{r}, E, t) \rightarrow \infty$ by Eq. (A.3). Therefore, $\Lambda(t) \rightarrow 0$ by Eq. (A.7b) and $C_{i}(t)$, which is proportional to the normalization of $S(\bar{r}, E, t)$ by Eq. (A. $4 b)$, becomes large. Then all terms divided by $\Lambda(t)$ or containing $C_{i}(t)$ in the point kinetics equations, Eqs. (A.8), are large compared to $\frac{\partial \phi(t)}{\partial t}$. Thus if $\frac{1}{V(E)} \rightarrow 0$, then 
Eqs. (A.8) become the prompt jump equations:

$$
\begin{gathered}
0=\frac{\rho(t)-\beta}{\Lambda(t)} \phi(t)+\sum_{i=1}^{I} \lambda_{i} C_{i}(t) \\
\frac{\partial C_{i}(t)}{\partial t}=\frac{\beta_{i}}{\Lambda(t)} \phi(t)-\lambda_{i} C_{i}(t) \quad i=1,2, \cdots, I
\end{gathered}
$$

Also, note that if more than one material composition is considered then $\beta_{i}$ of Eqs. (A.8) may be time dependent and slightly different from $\beta_{i}$ of Eq. (A.I) ${ }^{15}$.

Whereas no approximations have been made in deriving Eqs. (A.8), the point kinetics equations are as accurate as the diffusion equation. However, the point kinetics parameters cannot be determined unless $S(\bar{r}, E, t)$ is known. The shape function must be either approximated or calculated from the time, energy, and space dependent neutron diffusion equation. This latter approach is self defeating since the purpose of the point kinetics equations is to avoid this calculation. Hence $S(\bar{r}, E, t)$ is usually approximated by the steady state solution of Eqs. (A.1). (When this is done the initial adjoint flux shape is generally used as a weight function in deriving the point kinetics parameters. Errors in the necessarily approximate values of $\rho(t)$ and $\Lambda(t)$ are thereby reduced.) When a constant 
shape function is used to determine the point kinetics parameters, the point kinetics equations are easily solved. However, the point kinetics solution is no longer as accurate as the diffusion equation solution except for homogeneous reactor problems in which case $S(\vec{r}, E, t)$ is, in fact, time independent.

\section{A.3 The Multigroup Neutron Diffusion Equations}

The multigroup form of the neutron diffusion equation, Eq. (A.I), is obtained by partitioning the continuous energy range into energy groups. In order to define $G$ energy groups in the range of energies between $E_{G}=0$ and $E_{0}=E_{\max }, G$ energy group boundaries, $E_{g}$, are chosen such that

$$
E_{\max }=E_{0}>E_{1}>E_{2}>\ldots>E_{g}>\ldots>E_{G-1}>E_{G}=0
$$

Then any energy, E, such that

$$
E_{g}<E<E_{g-1}
$$

is said to be in energy group $g$. Once the energy groups are defined the multigroup neutron diffusion equations are derived by integrating Eq. (A.I) over each energy group and then defining multigroup parameters: 
166

$$
\begin{aligned}
& \frac{\partial}{\partial t} \int_{E_{g}}^{E_{g-1}} \frac{1}{v(E)} \phi(\bar{r}, E, t) \\
& \quad=\int_{E_{g}}^{E_{g-1}}\left[\bar{\nabla} \cdot D(\bar{r}, E, t) \nabla \phi(\bar{r}, E, t)-\Sigma_{t}(\bar{r}, E, t) \phi(\bar{r}, E, t)\right] d E \\
& +\int_{E_{g}}^{E_{g-1}} \sum_{g^{\prime}=1}^{G} \int_{E_{g^{\prime}}}^{E_{g^{\prime}-1}}\left[(1-\beta) X(E) \nu \Sigma_{f}\left(\bar{r}, E^{\prime}, t\right)+\Sigma_{s}\left(\bar{r}, E^{\prime} \rightarrow E, t\right)\right] \phi\left(\bar{r}, E^{\prime}, t\right) d E^{\prime} d E \\
& +\sum_{i=1}^{I} \lambda_{i} C_{i}(\bar{r}, t) \int_{E_{g}}^{E_{g-1}} f_{i}(E) d E \quad . \\
& \quad \quad(A .10)
\end{aligned}
$$

The multigroup flux is defined as

$$
\phi_{g}(\bar{r}, t) \equiv \int_{E_{g}}^{E_{g-1}} \phi(\vec{r}, E, t) d E
$$

It is supposed that a multigroup diffusion coefficient, $D_{g}(\bar{r}, t)$, may be found such that

$$
D_{g}(\bar{r}, t) \nabla \phi_{g}(\bar{r}, t)=\int_{E_{g}}^{E_{g-1}} D(\bar{r}, E, t) \dot{\nabla} \phi(\bar{r}, E, t) d E
$$

In practice, $D_{g}(\bar{r}, t)$ may be found by assuming a flux of the form 


$$
\phi(\bar{r}, E, t)=R(\bar{r}, t) \xi(E)
$$

Then,

$$
D_{g}(\bar{r}, t) \equiv \frac{\int_{E_{g}}^{E_{g-1}} D(\bar{r}, E, t) \xi(E) d E}{\int_{E_{g}}^{E_{g-1}} \xi(E) d E}
$$

The remaining multigroup constants are defined as:

$$
\begin{aligned}
& \Sigma_{t g}(\bar{r}, t) \equiv \int_{E_{g}}^{E_{g-1}} \Sigma_{t}(\bar{r}, E, t) \phi(\bar{r}, E, t) d E / \phi_{g}(\bar{r}, t) \\
& \Sigma_{s g g^{\prime}}(\bar{r}, t) \equiv \int_{E_{g}}^{E_{g-1}} \int_{E_{g^{\prime}}}^{E_{g^{\prime}-1}} \Sigma_{s}\left(\bar{r}, E^{\prime} \rightarrow E, t\right) \phi\left(r, E^{\prime}, t\right) d E^{\prime} d E / \phi_{g}(\bar{r}, t) \\
& \nu \Sigma_{f_{g}}(\bar{r}, t) \equiv \int_{E_{g}}^{E_{g-1}} \nu \Sigma_{f}(\bar{r}, E, t) \phi(\bar{r}, E, t) d E / \phi_{g}(\bar{r}, t) \\
& X_{g} \equiv \int_{E_{g}}^{E_{g-1}} X(E) d E \\
& f_{g i} \equiv \int_{E_{g}}^{E_{g-1}} f_{i}(E) d E \quad i=1,2, \cdots, I \\
& \frac{1}{V_{g}} \equiv \int_{E_{g}}^{E_{g-1}} \frac{1}{v(E)} \phi(\bar{r}, E, t) d E / \phi_{g}(\bar{r}, t)
\end{aligned}
$$


The above multigroup constants are considered to be known quantities. Using these definitions in Eqs. (A.10) and (A.Ib) leads to the multigroup neutron diffusion equations:

$$
\begin{aligned}
& \frac{1}{v_{g}} \frac{\partial \phi_{g}(\bar{r}, t)}{\partial t}=\bar{\nabla} \cdot D_{g}(\bar{r}, t) \nabla \phi_{g}(\bar{r}, t)-\sum_{t g}(\bar{r}, t) \phi_{g}(\bar{r}, t) \\
& +\sum_{g^{\prime}=1}^{G}\left[(1-\beta) X_{g^{\nu}} \Sigma_{f_{g^{\prime}}}(\bar{r}, t)+\Sigma_{s g^{\prime}}(\bar{r}, t)\right] \phi_{g^{\prime}}(\bar{r}, t) \\
& +\sum_{i=1}^{I} f_{g i} \lambda_{i} C_{i}(\bar{r}, t) \\
& 9=1,2, \cdots, G \\
& \begin{array}{r}
\frac{\partial C_{\lambda}(\bar{r}, t)}{\partial t}=\sum_{g=1}^{G} \beta_{i} \nu \Sigma_{f g}(\bar{r}, t) \phi_{g}(\bar{r}, t)-\lambda_{i} C_{i}(\bar{r}, t) \\
i=1,2, \cdots, I
\end{array}
\end{aligned}
$$

A.3a The One-Group, One-Dimension Neutron Diffusion Equation For one energy group,

$$
X_{g}=1 \quad ; \quad f_{g i}=1 \quad i=1,2, \cdots, I
$$

and

$$
\sum_{s_{g g^{\prime}}}(\bar{r}, t)=\sum_{t g}(\bar{r}, t)-\sum_{a_{g}}(\bar{r}, t) \underbrace{}_{g=g^{\prime}=1}
$$

where $\Sigma_{a g}(\bar{r}, t)$ is the absorption cross section. Therefore, the one group neutron diffusion equation is 


$$
\begin{aligned}
\frac{1}{v} \frac{\partial \phi}{\partial t}(\bar{r}, t) & =\bar{\nabla} \cdot D(\bar{r}, t) \nabla \phi(\bar{r}, t)-\sum_{a}(\bar{r}, t) \phi(\bar{r}, t) \\
+ & (1-\beta) \frac{\nu \Sigma_{f}}{K_{e f f}}(\bar{r}, t) \phi(\bar{r}, t)+\sum_{i=1}^{I} \lambda_{i} C_{i}(\bar{r}, t)
\end{aligned}
$$

Where $c_{i}(\bar{r}, t)$ is found by

$$
\begin{array}{r}
\frac{\partial C_{i}}{\partial t}(\bar{r}, t)=\beta_{i} \frac{\nu \Sigma_{f}}{k_{\text {eff }}}(\bar{r}, t) \phi(\bar{r}, t)-\lambda_{i} C_{i}(\bar{r}, t) \\
i=1,2, \cdots, I
\end{array}
$$

The $g$ subscript has been omitted because there is only one energy group. The multiplication factor, keff, has been added to adjust for criticality of the steady state equation if desired.

For one dimension, $\bar{\nabla} \cdot D(\bar{r}, t) \nabla \phi(\bar{r}, t)$ is replaced by $\frac{\partial}{\partial x} D(x, t) \frac{\partial}{\partial x} \phi(x, t)$ and $x$ replaces $\bar{r}$ in Eqs. (A.12). This yields the one-group, one-dimension neutron diffusion equation:

$$
\begin{aligned}
\frac{1}{v} \frac{\partial}{\partial t} \phi(x, t) & =\frac{\partial}{\partial x} D(x, t) \frac{\partial}{\partial x} \phi(x, t)-\Sigma_{a}(x, t) \phi(x, t) \\
& +(1-\beta) \frac{\nu \Sigma_{f}}{k_{e f f}}(x, t) \phi(x, t)+\sum_{i=1}^{I} \lambda_{i} C_{i}(x, t)
\end{aligned}
$$




$$
\begin{array}{r}
\frac{\partial C_{i}(x, t)}{\partial t}=\beta_{i} \frac{\nu \Sigma_{f}(x, t)}{k_{\text {eff }}} \phi(x, t)-\lambda_{i} C_{i}(x, t) \\
i=1,2, \cdots, I
\end{array}
$$

\section{A. 3b An Alternative Form of the Multigroup Neutron Diffusion Equations}

Eqs. (A.II) may be compacted by making the following definitions :

$\Sigma_{g g^{\prime}}(\bar{r}, t) \equiv(1-B) X_{g} \nu \Sigma_{f g^{\prime}(\bar{r}, t)+\Sigma_{s g g^{\prime}}(\bar{r}, t) \quad g_{g^{\prime}} \neq g}$

$\Sigma_{g g}(\bar{r}, t) \equiv(1-\beta) X_{g} \nu \Sigma_{f g}(\bar{r}, t)-\Sigma_{t g}(\bar{r}, t)$

$p_{i g}(\bar{r}, t) \equiv \beta_{i} \nu \Sigma_{f_{g}}(\bar{r}, t)$

These definitions lead to the following alternate form of the multigroup neutron diffusion equations:

$$
\begin{aligned}
& \frac{1}{V_{g}} \frac{\partial \phi_{g}(\bar{r}, t)=}{\partial t}=\bar{\nabla} \cdot D_{g}(\bar{r}, t) \nabla \phi_{g}(\bar{r}, t)+\sum_{g^{\prime}=1}^{G} \sum_{g g^{\prime}}(\bar{r}, t) \phi_{g^{\prime}}(\bar{r}, t) \\
&+\sum_{i=1}^{I} f_{g^{i}} C_{i}(\bar{r}, t) \quad
\end{aligned}
$$

$$
\begin{array}{r}
\frac{\partial C_{i}(\bar{r}, t)}{\partial t}=\sum_{g=1}^{G} P_{i g}(\bar{r}, t) \phi_{g}(\bar{r}, t)-\lambda_{i} C_{i}(\bar{r}, t) \\
i=1,2, \cdots, I
\end{array}
$$


A. 4 The A-Matrix and Flux Vector

The fully discretized finite difference form of the multigroup neutron diffusion equations, Eqs. (A.14), may be written in matrix form as in Eq. (1.6) of Chapter 1:

$$
\frac{\bar{\psi}^{n+1}-\bar{\psi}^{n}}{h}=\underline{A}_{1} \bar{\psi}^{n}+\underline{A}_{2} \bar{\psi}^{n+1}
$$

where $\underline{A}_{1}+\underline{A}_{2}=\underline{A}$. Several forms of $\underline{A}_{\text {and }} \bar{\psi}^{\mathrm{n}}$ are possible depending upon the ordering of unknowns. In this section, the A-matrix and flux vector, $\psi^{n}$, used in the MITKIN and ADC codes will be described, but first it is useful to mention the treatment of diffusion terms and boundary conditions.

In two-dimensional rectangular geometry the diffusion term of Eq. (A.14a) may be represented by a five point central difference approximation. For three-dimensions a seven point formula is used and for one-dimension a three point formula is used. As an example, consider the two dimensional form,

$$
\begin{aligned}
& \left.\bar{\nabla} \cdot D_{g}(\bar{r}, t) \nabla \phi_{g}(\bar{r}, t)\right|_{\bar{r}=\bar{r}_{k j}} \\
& =D_{g}\left[\frac{\phi_{g, k-1, j}^{n}-2 \phi_{g, k, j}^{n}+\phi_{g, k+1, j}^{n}}{h_{x}^{2}}+\frac{\phi_{g, k, j-1}^{n}-2 \phi_{g, k, j}^{n}+\phi_{g, k, j+1}^{n}}{h_{4}^{2}}\right]_{(A .15)}
\end{aligned}
$$


where

$$
\begin{aligned}
& \mathrm{n}=\text { time step number } \\
& \mathrm{k}=\mathrm{x} \text {-direction mesh index } \\
& j=y \text {-direction mesh index } \\
& h_{x}=x \text {-direction mesh spacing } \\
& h_{y}=y \text {-direction mesh spacing } \\
& \bar{r}_{k j}=\text { spatial location of mesh point }(k, j) \\
& D_{g}=\text { gth energy group diffusion coefficient, } \\
& \text { assumed to be spatially constant for } \\
& \text { simplicity. For a spatially dependent } \\
& \text { diffusion coefficient, Eq. (A.15) becomes }
\end{aligned}
$$

Both the MITKIN and ADC codes use a five point formula like that of Eq: (A.15). The consequence of this is that the A-matrix is penta-block diagonal. (The WIGLPJ code uses the corresponding one-dimensional three point formula with a resulting tri-block diagonal A-matrix.)

As for the treatment of boundary conditions, all boundary'fluxes are set to zero; that is, homogeneous Dirichlet boundary conditions are used.

It is now possible to illustrate the A-matrix and flux vector, $\Psi^{n}$, of the MITKIN and ADC codes: 
173

$$
A \bar{\psi}^{n}=
$$

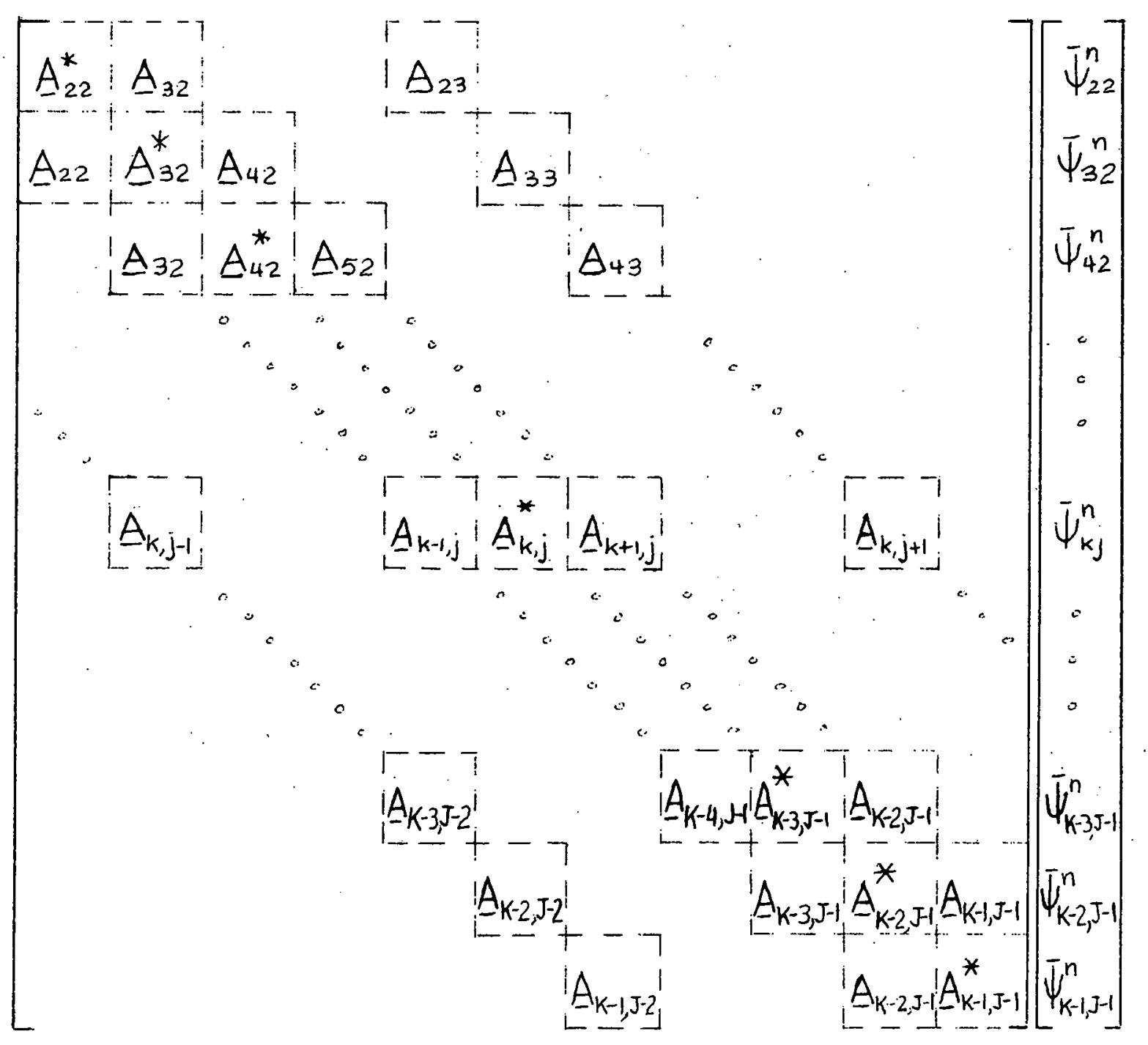


Each subvector, $\bar{\psi}_{k j}^{n}$, of $\bar{\psi}^{n}$ has G+I elements and represents both fluxes and precursors for all neutron and precursor groups at a given spatial mesh point, $(k, j)$.

$$
\bar{\psi}_{k j}^{n}=\left[\begin{array}{l}
\phi_{I k j}^{n} \\
\vdots \\
\phi_{g k j}^{n} \\
\vdots \\
\phi_{G k j}^{n} \\
c_{I k j}^{n} \\
\vdots \\
c_{I k j}^{n}
\end{array}\right]
$$

Each submatrix $\underline{A}_{k, j-1}, \underline{A}_{k-1, j}, \underline{A}_{k+1, j}$, and $\underline{A}_{k, j-1}$ is a $G+I$ diagonal matrix. The first $G$ diagonal elements are the diffusion terms, $\frac{v_{g} D_{g}}{h_{x}^{2}}$ or $\frac{v_{g} D_{g}}{h_{y}^{2}}$, associated with the mesh point of the coefficient flux vector; the remaining I elements are zeros. Only the block diagonal submatrices $A_{-k j}^{*}$ are dense. 


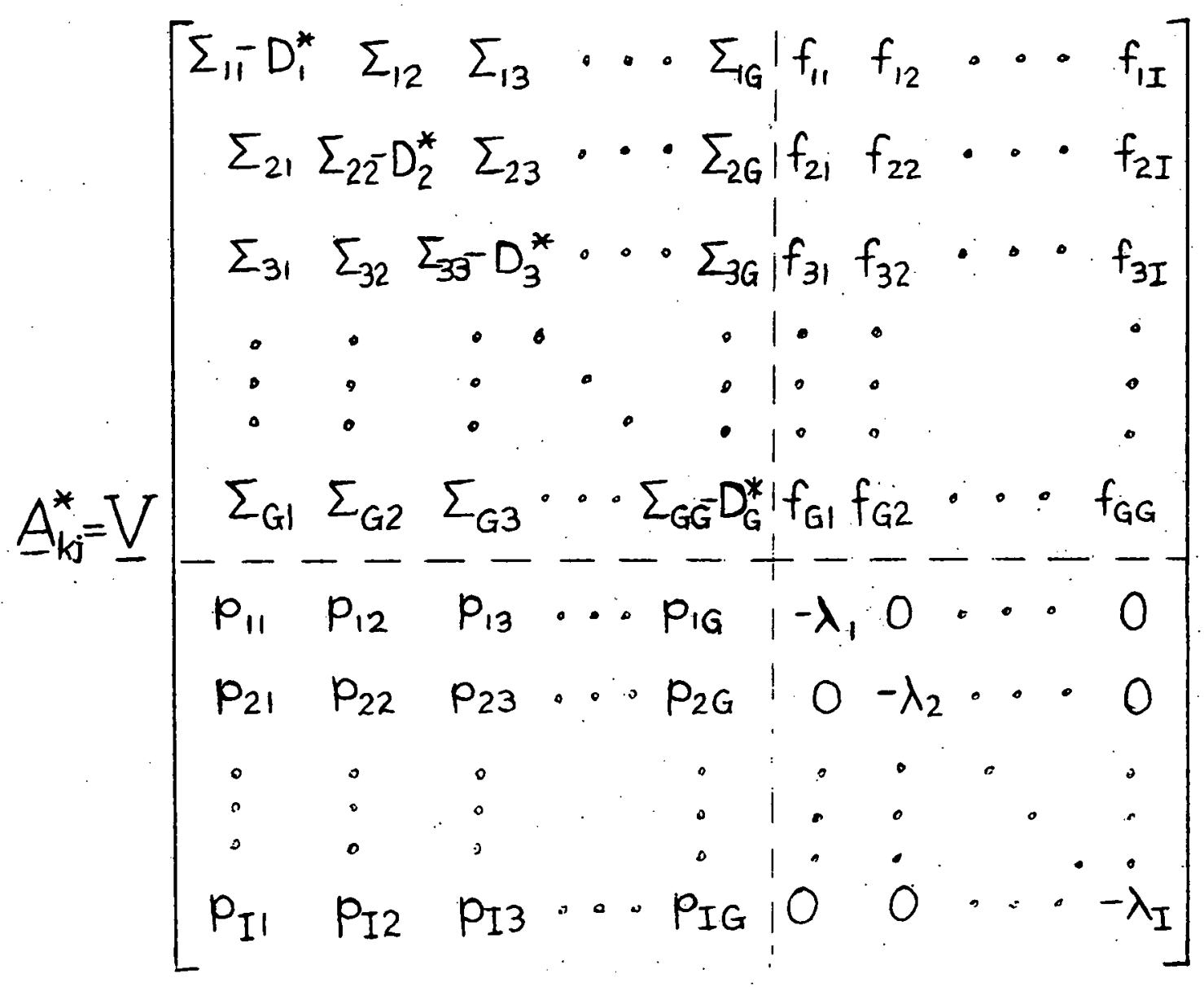

Here, $\mathrm{D}_{\mathrm{g}}^{*}=\frac{2 \mathrm{D}_{\mathrm{g}}}{\mathrm{h}_{\mathrm{x}}^{2}}+\frac{2 \mathrm{D}_{\mathrm{g}}}{\mathrm{h}_{\mathrm{y}}^{2}}$.

The elements $D_{g}, \quad \Sigma_{g g}{ }^{\prime}, f_{g i}$, and $p_{i g}$ are all associated with the mesh point $(k, j)$. These parameters take on averaged values at interfaces between different material regions. (Interfaces and boundaries run through mesh points rather than between them.) The velocity matrix, 
$\underline{V}$, is a G+I diagonal matrix, the first $G$ elements being the velocities $\mathrm{v}_{\mathrm{g}}$ and the final I elements being unity. The A-matrix, then, is a nonsymmetric, sparse, pentablock diagonal matrix with real elements. Off diagonal elements are all positive; the diagonal elements are negative for problems of physical interest. The A-matrix is "stiff" in that its eigenvalues range over many orders of magnitude. 


\section{Appendix B}

VECTOR AND MATRIX NORMS

Just as it is often useful to compare two vectors in terms of length, it is also often useful to compare matrices by some measure. Norms provide a convenient measure for vectors and matrices.

The norm of a real* vector $\bar{x}$ with $N$ elements, $x_{n}$, is defined as

$$
\|\bar{x}\|_{l} \equiv\left(\sum_{n=1}^{N}\left|x_{n}\right|\right)^{1 / l}
$$

where $l$ is an integer. For example, the $L_{2}$ norm $(l=2)$ of $\overline{\mathrm{x}}$ is

$$
\|\bar{x}\|_{2}=\left(\sum_{n=1}^{N}\left|x_{n}\right|^{2}\right)^{1 / 2}=\left(\bar{x}^{\top} \bar{x}\right)^{1 / 2}
$$

The norm of a real square matrix $\underline{A}$ is defined as

$$
\|\underline{A}\|_{l} \equiv \frac{\|\underline{A} \bar{x}\|_{l}}{\|\bar{x}\|_{l}}
$$

where $\dot{\mathrm{x}}$ is the nonzero real vector for which the ratio of vector norms, $\frac{\|\underline{A}\|_{l}}{\|\bar{x}\|_{l}}$, is maximized. For example, if $\overline{\mathrm{x}}$ is the real nonzero vector for which

* Only real vectors and matrices are considered in this thesis. 


$$
\frac{(A \bar{x})^{\top}(A \bar{x})}{\bar{x}^{\top} \bar{x}}
$$

is maximized, then the $\mathrm{L}_{2}$ norm of $\mathrm{A}$ is

$$
\|A\|_{2} \equiv\left\{\frac{(A \bar{x})^{\top}(A \bar{x})}{\bar{x}^{\top} \bar{x}}\right\}^{1 / 2}
$$

It can be shown 31 that if $\underline{A}$ is real and symmetric then $\rho$, the spectral radius $\underline{A}$, is bounded by all norms of $\underline{A}$ :

$$
p \leq\|A\|_{l} \quad l=1,2, \cdots, \infty
$$

(The spectral radius of a matrix is the largest characteristic value (eigenvalue) of that matrix.) Furthermore, it can be shown 31 that if any norm of a matrix $\underline{A}$, say the $L_{n}$ norm, $\|A\|_{n}$, is bounded, then all norms of $\underline{A}$, $\|A\|_{l} \quad l=1,2, \ldots, \infty$ are bounded.

Vector and matrix norms have the following properties which should be obvious from the definitions:*

1) $\|\bar{x}\| \geqslant 0 ; \quad\|\underline{A}\| \geqslant 0$ $\|\bar{x}\|=0$ only if all elements of $\bar{x}$ are zero $\|A\|=0$ only if all elements of $\underline{A}$ are zero

2) $\|\alpha \bar{x}\|=\alpha\|\bar{x}\| ; \quad\|\alpha \underline{A}\|=\alpha\|A\|, \quad \alpha=$ constant

* The norm subscript, $l$, is omitted for all properties independent of $l$. 
179

3) $\|\bar{x}+\bar{y}\| \leqslant\|\bar{x}\|+\|\bar{y}\| ;\|\underline{A}+\underline{B}\| \leqslant\|\underline{A}\|+\|\underline{B}\|$

Additionally, for matrix norms,

4) $\|\underline{A} \underline{B}\| \leqslant\|\underline{A}\|\|\underline{B}\|$.

This fourth property is not as readily apparent as the first three properties. Therefore, it is now proven.

Proof of property 4:

(The proof is carried out in the $\mathrm{L}_{2}$ norm for simplicity although any other norms can be used.)

Let $\bar{u}, \bar{v}$, and $\bar{w}$ be the n-element nonzero real vectors for which

$\frac{(A \bar{u})^{\top}(A \bar{u})}{\bar{u}^{\top} \bar{u}}, \frac{(\underline{B} \bar{v})^{\top}(B \bar{v})}{\bar{v}^{\top} \bar{v}}$ and $\frac{(\underline{B} \underline{\underline{w}})^{\top}(\underline{A} \underline{\underline{w}})}{\bar{w}^{\top} \bar{w}}$

are respectively maximized.

Let $\bar{z}=\underline{B} \bar{w}$

Now,

$$
\left\{\frac{(\underline{A} \bar{z})^{\top}(\underline{A} \bar{z})}{\bar{z}^{\top} \bar{z}}\right\}^{1 / 2} \leq\left\{\frac{(\underline{A} \bar{u})^{\top}(\underline{A} \bar{u})}{\bar{u}^{\top} \bar{u}}\right\} \equiv\|\underline{A}\|_{2} .
$$

and

$$
\left\{\frac{(\underline{B} \bar{w})^{\top}(B \bar{w})}{\bar{w}^{\top} \bar{w}}\right\}^{1 / 2} \leqslant\left\{\frac{(\underline{B} \bar{v})^{\top}(\underline{B} \bar{v})}{\bar{v}^{\top} \bar{v}}\right\} \equiv\|\underline{B}\|_{2}
$$


180

Therefore,

$$
\begin{aligned}
\|\underline{A} \underline{B}\|_{2} & \equiv\left\{\frac{(\underline{A} \underline{\underline{B}} \bar{w})^{\top}(\underline{A} \underline{B} \bar{w})}{\bar{w}^{\top} \bar{w}}\right\}^{1 / 2} \\
& =\left\{\frac{(A \bar{z})^{\top}(A \bar{z})}{\bar{w}^{\top} \bar{w}}\right\}^{1 / 2}
\end{aligned}
$$

from Eq. (B.2)

$$
\begin{aligned}
& =\left\{\frac{(A \bar{z})^{\top}(\underline{A} \bar{z})}{\bar{z}^{\top} \bar{z}}\right\}^{1 / 2}\left\{\frac{\bar{z}^{\top} \bar{z}}{\bar{w}^{\top} \bar{w}}\right\}^{1 / 2} \\
& =\left\{\frac{(\underline{A} \bar{z})^{\top}(\underline{A} \bar{z})}{\bar{z}^{\top} \bar{z}}\right\}^{1 / 2}\left\{\frac{(\underline{B} \bar{w})^{\top}(\underline{B} \bar{w})}{\bar{w}^{\top} \bar{w}}\right\}^{1 / 2}
\end{aligned}
$$

Finally, from Ens. (B.2) and (B.3),

$$
\text { Q.E.D. }
$$

$$
\|\underline{A} \underline{B}\|_{2} \leqslant\|\underline{A}\|_{2}\|\underline{B}\|_{2}
$$

Matrix and vector norms are very useful in the mathematical analysis of numerical properties. In particular, stability may be defined in terms of a matrix norm as follows: 31

Definition of numerical stability:

The system of equations

$$
\bar{x}\left(t^{n+2}\right)=\underline{A} \bar{x}\left(t^{n}\right)
$$


181

or equivalently

$$
\bar{x}\left(t^{2 m}\right)=A^{m} \bar{x}\left(t^{\circ}\right) \quad 2 m=n+2
$$

is numerically stable for $t^{0} \leq t \leq t^{2 m}$ if $\left\|A^{m}\right\|$ is bounded by some constant independent of $\mathrm{m}$ :

$$
\left\|A^{m}\right\|<K \text {, a constant independent of } \mathrm{m} \text {. }
$$




\section{Appendix C \\ SUPPLEMENTARY STABILITY ANALYSIS}

In Section $3.3 \mathrm{c}$ it was stated without proof that the advancement matrix, $\underline{B}$, of the alternating direction method difference system,

$$
\bar{\psi}^{n+2}=\underline{B} \psi^{n}
$$

may be factored as

$$
\underline{B}=\left\{\left[\underline{I}+h \underline{C}_{1}\right]^{-1}\left[\underline{I}-h \underline{C}_{2}\right]\left[\underline{I}+h \underline{C}_{2}\right]^{-1}\left[\underline{I}-h \underline{C}_{1}\right]+\underline{Q}(h)\right\}
$$

where $\left(\underline{C}_{1}+\underline{C}_{2}\right)=\underline{C}$ is the principal part of the finite difference neutron diffusion equation $\underline{A}$-matrix and where $\underline{Q}(\mathrm{~h})$ is a bounded family of operators. The truth of this statement will now be demonstrated for ADI, ADE, and checkerboard methods with the two-level frequency transform alternating direction method advancement matrix,

$$
\underline{B}=\left[\underline{I}-h\left(\underline{A}_{4}-\alpha \underline{\Omega}\right)\right]^{-1} e^{\underline{\Omega} h}\left[\underline{I}+h\left(\underline{A}_{3}-\gamma \underline{\Omega}\right)\right]\left[\underline{I}-h\left(\underline{A}_{2}-\alpha \underline{\Omega}\right)\right]^{-1} e^{\underline{\Omega} h}\left[\underline{I}+h\left(\underline{A_{1}}-\gamma \underline{\Omega}\right)\right]
$$

The following three theorems will be useful:

Theorem C.I If the real nxn matrix $\left(\underline{P}_{k}^{\top}+\underline{P}_{K}\right)$ is positive definite, then $\left(\underline{I}+h \underline{\underline{P}}_{k}\right)^{-1}$ is a bounded operator with $\mathrm{L}_{2}$ norm

$$
\left\|\left(\underline{I}+h \underline{P}_{k}\right)^{-1}\right\|_{2} \leqslant 1
$$


Proof:

Let $\bar{x}$ be the real n-element nonzero vector for which

$$
\frac{\left[\left(I+h \underline{P}_{K}\right)^{-1} \bar{x}\right]^{\top}\left[\left(I+h \underline{P}_{K}\right)^{-1} \bar{x}\right]}{\bar{x}^{\top} \bar{x}}
$$

is maximized. Also, let

$$
\bar{v}=\left(\underline{I}+h \underline{P}_{K}\right)^{-1} \bar{x}
$$

Note that $\bar{v}^{\top} \bar{v} \geqslant 0$ and $\left(\underline{P}_{K} \bar{v}\right)^{\top}\left(\underline{P}_{K} \bar{v}\right) \geqslant 0$ because the product of a real vector and its transpose is a positive number. Also, $\bar{v}^{\top}\left(\underline{P}_{K}^{\top}+\underline{P}_{K}\right) \bar{v} \geqslant 0$ because. $\left(\underline{P}_{K}^{\top}+\underline{P}_{K}\right)$ is positive definite.

Therefore,

$$
\begin{aligned}
& \left\|\left(\underline{I}+h \underline{\underline{P}}_{k}\right)^{-1}\right\|_{2} \equiv\left\{\frac{\left[\left(\underline{I}+h \underline{\underline{P}}_{k}\right)^{-1} \bar{x}\right]^{\top}\left[\left(\underline{I}+h \underline{P}_{k}\right)^{-1} \bar{x}\right]}{\bar{x}^{\top} \bar{x}}\right\}^{1 / 2} \\
& =\left\{\frac{\bar{v}^{\top} \bar{v}}{\left[\left(I+h \underline{P}_{K}\right) \bar{v}\right]^{\top}\left[\left(\underline{I}+h \underline{P}_{K}\right) \bar{v}\right]}\right\}^{1 / 2} \\
& =\left\{\frac{\bar{v}^{\top} \bar{v}}{\bar{v}^{\top} \bar{v}+h \bar{v}^{\top}\left(\underline{\underline{P}}_{K}^{\top}+\underline{\underline{P}}_{K}\right) \bar{v}+h^{2}\left(\underline{P}_{k} \bar{v}\right)^{\top}\left(\underline{P}_{k} \bar{v}\right)}\right\}^{1 / 2} \\
& \leq 1
\end{aligned}
$$

Q.E.D. 
Theorem C.2: If the real nxn matrix $\subseteq_{k}$ may be factored as

$$
\underline{C}_{k}=\underline{F}_{k} \underline{H}
$$

where $\underline{F}_{k}$ and $\underline{H}$ are positive definite and symmetric so that $E_{K}=E_{K}^{T / 2} E_{K}^{1 / 2} ; \quad \underline{H}=\underline{H}^{T / 2} \underline{H}^{1 / 2} ;$ and $\left\|H^{1 / 2}\right\|_{2}$ and $\left\|H^{-\frac{1}{2}}\right\|_{2}$ are bounded, then $\left\|\left(\underline{I}+h \underline{C}_{k}\right)^{-1}\right\|_{2}$ is a bounded operator such that

$$
\left\|\left(\underline{I}+h \underline{C}_{K}\right)^{-1}\right\|_{2} \leqslant K \text {, a constant }
$$

Proof:

$$
\begin{aligned}
\left(\underline{I}+h \underline{C}_{k}\right)^{-1} & =\left(\underline{I}+h \underline{F}_{k} \cdot \underline{H}\right)^{-1} \\
& =\left[\underline{H}^{-1 / 2}\left(\underline{I}+h \underline{H}^{1 / 2} \underline{F}_{k} \underline{H}^{T / 2}\right) \underline{H}^{1 / 2}\right]^{-1} \\
& =\underline{H}^{-1 / 2}\left(\underline{I}+h \underline{H}^{1 / 2} \underline{F}_{k} \underline{H}^{T / 2}\right)^{-1} \underline{H}^{1 / 2}
\end{aligned}
$$

Now, let $\underline{P}_{k} \equiv H^{1 / 2} E_{K} \underline{H}^{T / 2}$. and let $\bar{u}$ be any real n-element vector. Then

$$
\begin{aligned}
\bar{u}^{\top}\left(\underline{P}_{k}^{\top}+\underline{P}_{k}\right) \bar{u} & =\bar{u}^{\top}\left[\left(\underline{H}^{1 / 2} \underline{F}_{k} \underline{H}^{T / 2}\right)^{T}+\left(\underline{H}^{1 / 2} \underline{F}_{k} \underline{H}^{T / 2}\right)\right] \bar{u} \\
& =\bar{u}^{\top}\left[\left(\underline{H}^{1 / 2} \underline{E}_{k}^{T / 2} \underline{F}_{k}^{1 / 2} \underline{H}^{T / 2}\right)^{\top}+\left(\underline{H}^{1 / 2} \underline{F}_{k}^{T / 2} \underline{F}_{k}^{1 / 2} \underline{H}^{T / 2}\right)\right] \bar{u} \\
& =\bar{u}^{\top}\left[\left(\underline{F}_{k}^{1 / 2} \underline{H}^{T / 2}\right)^{T}\left(\underline{F}_{k}^{1 / 2} \underline{H}^{T / 2}\right)+\left(\underline{F}_{k}^{1 / 2} \underline{H}^{T / 2}\right)^{\top}\left(\underline{F}_{k}^{1 / 2} \underline{H}^{T / 2}\right)\right] \bar{u}
\end{aligned}
$$




$$
\begin{aligned}
\bar{u}^{\top}\left(\underline{P}_{k}^{\top}+\underline{P}_{k}\right) \bar{u} & =2 \bar{u}^{\top}\left(\underline{F}_{k}^{1 / 2} \underline{H}^{T / 2}\right)^{\top}\left(\underline{F}_{k}^{1 / 2} \underline{H}^{T / 2}\right) \bar{u} \\
& =2\left(\underline{F}_{k}^{1 / 2} \underline{H}^{T / 2} \bar{u}\right)^{\top}\left(\underline{F}_{k}^{1 / 2} \underline{H}^{T / 2} \bar{u}\right) \\
& =\underset{\text { the product of a real vector and its }}{\text { transpose }} \\
& \geqslant 0
\end{aligned}
$$

Therefore, $\left(\underline{P}_{k}^{\top}+\underline{P}_{k}\right)$ is positive definite and

$$
\left\|\left(\underline{I}+h \underline{P}_{k}\right)^{-1}\right\|_{2} \leqslant 1
$$

by Theorem C.I. Thus,

$$
\begin{aligned}
\left\|\left(\underline{I}+h \underline{C}_{K}\right)^{-1}\right\|_{2} & =\left\|\underline{H}^{-1 / 2}\left(\underline{I}+h \underline{H}^{1 / 2} \underline{F}_{K} \underline{H}^{T / 2}\right)^{-1} \underline{H}^{1 / 2}\right\|_{2} \\
& =\left\|\underline{H}^{-1 / 2}\left(\underline{I}+h \underline{P}_{K}\right)^{-1} \underline{H}^{1 / 2}\right\|_{2} \\
& \leq\left\|\underline{H}^{-1 / 2}\right\|_{2}\left\|\left(\underline{I}+h \underline{P}_{K}\right)^{-1}\right\|_{2}\left\|\underline{H}^{1 / 2}\right\|_{2} \\
& \leq\left\|\underline{H}^{-1 / 2}\right\|_{2}\left\|\underline{H}^{1 / 2}\right\|_{2} \\
& \leq K, \text { a constant }
\end{aligned}
$$

Q.E.D.

Theorem C.3: A family of matrices $\underline{M}_{n}$ of varying dimension nxn having at most $l<n$ nonzero elements in each row or column, $\ell$ being constant for all $n$, is uniformly bounded 
if the individual elements of the matrices $\underline{M}_{n}$ are uniformly bounded for all $\mathrm{n}$.

Proof:

Whereas all elements, $m_{j k}^{n}$, of the nxn matrices $\underline{M}_{n}$ are uniformly bounded as

$$
\left|m_{j k}^{n}\right|<C \text {, a constant }
$$

then

$$
\sum_{j=1}^{n}\left|m_{j k}^{n}\right| \leqslant c l \quad \text { for alz } k \quad \text { (c.la) }
$$

and

$$
\sum_{k=1}^{n}\left|m_{j k}^{n}\right| \leq C l \quad \text { for all } j \quad \text { (c.lb) }
$$

Also, if $x_{i}$ and $y_{i}$ are elements of the n-element vectors $\bar{x}$ and $\bar{y}$, respectively, then

$$
\left|\sum_{i=1}^{n} x_{i} y_{i}\right| \leqslant\left(\sum_{i=1}^{n} x_{i}^{2}\right)^{1 / 2}\left(\sum_{i=1}^{n} y_{i}^{2}\right)^{1 / 2}
$$

This is the Schwarz Inequality 16 .

Let $\bar{x}$ be the n-element real nonzero vector for which

$$
\frac{\left(\underline{M}_{n} \bar{x}\right)^{\top}\left(\underline{M}_{n} \bar{x}\right)}{\bar{x}^{\top} \bar{x}}
$$


187

is maximized and let the elements of $\bar{x}$ be $x_{1}, x_{2}, \ldots, x_{n}$.

Then,

$$
\begin{aligned}
& \left\|\underline{M}_{n}\right\|_{2} \equiv\left\{\frac{\left(\underline{M}_{n} \bar{x}\right)^{\top}\left(\underline{M}_{n} \bar{x}\right)}{\bar{x}^{\top} \bar{x}}\right\}^{1 / 2} \\
& =\left\{\frac{\sum_{i=1}^{n} \sum_{j=1}^{n} \sum_{k=1}^{n} x_{i} m_{k i}^{n} m_{k j}^{n} x_{j}}{\sum_{i=1}^{n} x_{i}^{2}}\right\} \\
& =\left\{\frac{\sum_{k=1}^{n}\left|\sum_{i=1}^{n}\left(m_{k i}^{n} x_{i}\right)\right|\left|\sum_{j=1}^{n}\left(m_{k j}^{n} x_{j}\right)\right|}{\sum_{i=1}^{n} x_{i}^{2}}\right\} \\
& =\left\{\frac{\sum_{k=1}^{n}\left|\sum_{i=1}^{n} m_{k i}^{n} x_{i}\right|^{2}}{\sum_{i=1}^{n} x_{i}^{2}}\right\}^{1 / 2} \\
& =\left\{\frac{\left.\left.\sum_{k=1}^{n}\left|\sum_{i=1}^{n}\right| m_{k i}^{n}\right|^{1 / 2}\left[\left|m_{k i}^{n}\right|^{1 / 2} x_{i}\right]\right|^{2}}{\sum_{i=1}^{n} x_{i}^{2}}\right\}^{1 / 2}
\end{aligned}
$$

by Eq. (C.1c),

$$
\leq\left\{\frac{\sum_{k=1}^{n}\left[\sum_{i=1}^{n}\left|m_{k i}^{n}\right|\right]\left[\sum_{i=1}^{n}\left|m_{k i}^{n}\right| x_{i}^{2}\right]}{\sum_{i=1}^{n} x_{i}^{2}}\right\}^{1 / 2}
$$


188

by Eq. (C .Ib),

$$
\leqslant\left\{\frac{\operatorname{cl} \sum_{k=1}^{n} \sum_{i=1}^{n}\left|m_{k i}^{n}\right| x_{i}^{2}}{\sum_{i=1}^{n} x_{i}^{2}}\right\}^{1 / 2}
$$

$$
=\left\{\frac{\operatorname{cl} \sum_{i=1}^{n} x_{i}^{2}\left(\sum_{k=1}^{n}\left|m_{k i}^{n}\right|\right)}{\sum_{i=1}^{n} x_{i}^{2}}\right\}^{1 / 2}
$$

by Eq. (C.1a),

$$
\begin{aligned}
& \leq\left\{\frac{c l \sum_{i=1}^{n} x_{i}^{2} C l}{\sum_{i=1}^{n} x_{i}^{2}}\right\}^{1 / 2} \\
& =C l
\end{aligned}
$$

Therefore,

$$
\left\|\underline{M}_{n}\right\|_{2} \leqslant C l
$$

Q.E.D.

The alternating direction method advancement matrix. for the ADI, $A D E$, and checkerboard methods with the twolevel frequency transform is 
189

$$
\begin{aligned}
\underline{B}= & {\left[\underline{I}-h\left(\underline{A}_{4}-\alpha \Omega\right)\right]^{-1} e^{\Omega h}\left[I+h\left(\underline{A}_{3}-\gamma \Omega\right)\right] } \\
& \times\left[\underline{I}-h\left(\underline{A}_{2}-\alpha \Omega\right)\right]^{-1} e^{\Omega h}\left[\underline{I}+h\left(\underline{A}_{1}-\gamma \Omega\right)\right]
\end{aligned}
$$

This may be factored as

$$
\underline{B}=\underline{\Delta}_{1} \underline{\Delta}_{2}
$$

where

$$
\begin{aligned}
& \underline{\Delta}_{1}=\left[\underline{I}-h\left(\underline{A}_{4}-\alpha \underline{\Omega}\right)\right]^{-1} e^{\Omega h}\left[\underline{I}+h\left(\underline{A}_{3}-\gamma \underline{\Omega}\right)\right] \\
& \underline{\Delta}_{2}=\left[\underline{I}-h\left(\underline{A}_{2}-\alpha \Omega\right)\right]^{-1} e^{\underline{\Omega} h}\left[\underline{I}+h\left(\underline{A}_{1}-\gamma \Omega\right)\right]
\end{aligned}
$$

As in Section $3.3 \mathrm{c}$, the matrices $\underline{A}_{1}, \underline{A}_{2}, \underline{A}_{3}$ and $\underline{A}_{4}$ are

$$
\begin{aligned}
& \underline{A}_{1}=-\underline{C}_{1}+\underline{R}_{1} \\
& \underline{A}_{2}=-\underline{C}_{2}+\underline{R}_{2} \\
& \underline{A}_{3}=-\underline{C}_{2}+\underline{R}_{3} \\
& \underline{A}_{4}=-\underline{C}_{1}+\underline{R}_{4}
\end{aligned}
$$

$$
\text { where } \begin{aligned}
\underline{C}_{1} & =-\underline{D}_{1} ; \quad \underline{C}_{2}=-\underline{D}_{2} \text { for ADE methods } \\
\underline{C}_{1} & =-\underline{D}_{y} ; \quad \underline{C}_{2}=-\underline{D}_{\mathrm{x}} \text { for ADI methods } \\
\underline{C}_{1} & =-\underline{I}_{\mathrm{x}} \underline{D} ; \quad \underline{C}_{2}=-\underline{I}_{0} \underline{D} \text { for checkerboard methods }
\end{aligned}
$$

Making use of the expansions 


$$
e^{\Omega h}=\underline{I}+h \Omega+O\left(h^{2}\right)
$$

and

$$
(\underline{I}-h \underline{E})^{-1}=\underline{I}+h \underline{E}+h^{2} \underline{E}^{2}+\cdots
$$

(this latter expansion being valid only if the spectral radius of $\mathrm{hE}$ is less than unity) leads to

$$
\begin{aligned}
& \underline{\Delta}_{1}=\left[\underline{I}-h\left(-\underline{C}_{1}+\underline{B}_{4}-\alpha \underline{\Omega}\right)\right]^{-1}\left[\underline{I}+h \underline{\Omega}+O\left(h^{2}\right)\right]\left[\underline{I}+h\left(-\underline{C}_{2}+\underline{R}_{3}-\gamma \Omega \underline{\Omega}\right)\right] \\
& =\left\{\left[\underline{I}+h \underline{C}_{1}\right]\left[\underline{I}-h\left(\underline{I}+h \underline{C}_{i}\right)^{-1}\left(\underline{R}_{4}-\alpha \underline{\Omega}\right)\right]\right\}^{-1} \\
& \times\left[\underline{\underline{I}}+h \underline{\Omega}+O\left(h^{2}\right)\right]\left[\left(\underline{I}-h \underline{C}_{2}\right)+h\left(B_{3}-\gamma \Omega\right)\right] \\
& =\left[\underline{I}-h\left(\underline{I}+h \underline{C}_{1}\right)^{-1}\left(\underline{R}_{4}-\alpha \underline{\Omega}\right)\right]^{-1}\left[\underline{I}+h \underline{C}_{1}\right]^{-1} \\
& \times\left[\underline{I}+h \underline{\Omega}+O\left(h^{2}\right)\right]\left[\left(\underline{I}-h \underline{C}_{2}\right)+h\left(\underline{R}_{3}-\gamma \underline{\Omega}\right)\right] \\
& =\left[\underline{I}+h\left(\underline{I}+h \underline{C}_{1}\right)^{-1}\left(\underline{R}_{4}-\alpha \underline{\Omega}\right)+O\left(h^{2}\right)\right]\left[\underline{I}+h \underline{C}_{1}\right]^{-1} \\
& \times\left[I+h \Omega+O\left(h^{2}\right)\right]\left[\left(\underline{I}-h \underline{C}_{2}\right)+h\left(\underline{R}_{3}-\gamma \underline{\Omega}\right)\right] \\
& =\left[\underline{I}+h \underline{C}_{1}\right]^{-1}\left[\underline{I}-h \underline{C}_{2}\right]+h \underline{Q}_{1}(h)
\end{aligned}
$$


Similarly,

$$
\Delta_{2}=\left[I+h \underline{C}_{2}\right]^{-1}\left[I-h \underline{C}_{1}\right]+h \underline{Q}_{2}(h)
$$

Finally,

$$
\underline{B}=\underline{\Lambda}_{1} \underline{\Lambda}_{2}=\left[\underline{I}+h \underline{C}_{1}\right]^{-1}\left[\underline{I}-h \underline{C}_{2}\right]\left[\underline{I}+h \underline{C}_{2}\right]^{-1}\left[\underline{I}-h \underline{C}_{1}\right]+h \underline{Q}(h)
$$

The matrices which compose $\underline{Q}(h)$ are products and/or powers of $\left(B_{1}-\gamma \Omega\right),\left(\underline{R}_{2}-\alpha \underline{\Omega}\right),\left(\underline{R}_{3}-\gamma \Omega\right),\left(\underline{R}_{4}-\alpha \underline{\Omega}\right), \Omega,\left(\underline{I}-h \underline{C}_{1}\right),\left(\underline{I}-h \underline{C}_{2}\right),\left(\underline{I}+h \underline{C}_{1}\right)^{-1}$ and $\left(I+h \underline{C}_{2}\right)^{-1}$. The boundedness of $Q(h)$ follows from the fact that these matrix components are all bounded if it is assumed that $\Omega$ is bounded.

Because $\left(\underline{C}_{1}+\underline{C}_{1}^{\top}\right)$ and $\left(\underline{C}_{2}+\underline{C}_{2}^{\top}\right)$ are positive definite for $A D I$ and $A D E$ methods, $\left(\underline{I}+h \underline{C}_{1}\right)^{-1}$ and $\left(\underline{I}+h \underline{C}_{2}\right)^{-1}$ are bounded for these methods according to Theorem C.1. For checkerboard methods, it can be shown by Theorem C.3 that $\left\|\underline{H}^{1 / 2}\right\|_{2}$ and $\left\|\underline{H}^{-1 / 2}\right\|_{2}$ are bounded. (See section 3.3c, also.) Thus Theorem C.2 may be used to show that $\left(\underline{I}+h \underline{C}_{1}\right)^{-1}$ and $\left(\underline{I}+h \underline{C}_{2}\right)^{-1}$ are bounded for checkerboard methods.

All nonzero elements in the matrix $\underline{R}$ are independent of the temporal and spatial meshes and are therefore uniformly bounded. Hence all nonzero elements in the matrices $\underline{R}_{1}, \underline{R}_{2}, \underline{R}_{3}$ and $\underline{R}_{4}$ are uniformly bounded, regardless of the splitting technique. Furthermore, the number of nonzero elements in each row of these matrices is less than or equal to the number of prompt and delayed neutron groups. 
Therefore, according to Theorem C. $3, \underline{R}_{1}, \underline{R}_{2}, \underline{R}_{3}$ and $\underline{R}_{4}$ are uniformly bounded and because $\Omega$ is assumed to be bounded, $\left(\underline{R}_{1}-\gamma \underline{\Omega}\right),\left(\underline{R}_{2}-\alpha \underline{\Omega}\right),\left(\underline{R}_{3}-\gamma \underline{\Omega}\right)$ and $\left(\underline{R}_{4}-\alpha \underline{\Omega}\right)$ are uniformly bounded.

AlI nonzero elements in the matrices $\left(\underline{I}-\mathrm{h} \underline{C}_{1}\right)$ and $\left(\mathrm{I}-\mathrm{hC} \underline{-}_{2}\right)$ are independent of temporal and spatial meshes because the ratios

$$
r_{x}=\frac{h}{h_{x}^{2}} \quad ; \quad r_{y}=\frac{h}{h_{y}^{2}} \quad \therefore \text { etc. }
$$

are fixed by the consistency conditions. Because the matrix $\underline{C}=-\underline{D}$ is a $2 N+1$ stripe matrix (See Appendix A.4) where $\mathrm{N}$ is the number of spatial dimensions, the number of nonzero elements in each row of these matrices is less than or equal to $l=2 N+1$. Therefore, $\left(I-h \underline{C}_{1}\right)$. and ( $\left.\underline{I}-\mathrm{h} \underline{C}_{2}\right)$ are uniformly bounded according to Theorem C.3. Because all the matrices which form the matrix $\underline{Q}(h)$ are uniformly bounded, $\underline{Q}(h)$ is bounded. Therefore, the advancement matrix

$$
\begin{aligned}
\underline{B}= & {\left[\underline{I}-h\left(\underline{A}_{4}-\alpha \Omega\right)\right]^{-1} e^{\Omega h}\left[\underline{I}+h\left(\underline{A}_{3}-\gamma \Omega\right)\right] } \\
& \times\left[\underline{I}-h\left(\underline{A}_{2}-\alpha \Omega\right)\right]^{-1} e^{\Omega} h\left[\underline{I}+h\left(\underline{A}_{1}-\gamma \Omega\right)\right]
\end{aligned}
$$

of the difference system

$$
\bar{\psi}^{n+2}=\underline{B} \bar{\psi}^{n}
$$


may be factored as

$$
B=\left[\underline{I}+h \underline{C}_{1}\right]^{-1}\left[\underline{I}-h \underline{C}_{2}\right]\left[\underline{I}+h \underline{C}_{2}\right]^{-1}\left[\underline{I}-h \underline{C}_{1}\right]+h \underline{Q}(h)
$$

where $\underline{c}=\underline{c}_{1}+\underline{c}_{2}$ is the principal part of $\underline{A}$ and where $\underline{Q}(h)$ is a bounded family of operators. 
Appendix D

CRITICAL CONFIGURATIONS

This Appendix specifies the nuclear parameters defining the critical configurations of the reactor problems studied in this thesis. Notation is consistent with that of Appendix A.3 with arguments $(\bar{r}, t)$ omitted.

\section{Critical Configuration 1}

Geometry: Homogeneous, 1-dimension

Reactor width $=300 \mathrm{~cm} ; 3$ mesh spaces

1 precursor group:

$$
B=0.006 \quad \lambda=0.12
$$

1 neutron group:

$$
\begin{array}{rlrl}
v & =2 \times 105 & \Sigma_{a} & =0.1 \\
D & =5.0 & \nu \Sigma_{f}=0.1 \\
k_{\text {eff }} & =0.99502487562
\end{array}
$$

Initial conditions: cosine spatial shape

$$
\phi(0)=1.0 \quad C(0)=5.025 \times 10^{-3} \quad \begin{aligned}
& \text { measured at } \\
& \text { mesh } \# 2
\end{aligned}
$$

Critical Configuration 2

Geometry: Homogeneous, l-dimension

Reactor width $=300 \mathrm{~cm} ; 4$ mesh spaces

1 precursor group:

$$
\hat{\beta}=0.007 \quad \lambda=0.08
$$

1 neutron group:

$$
\begin{aligned}
D & =0.5 \quad \nu \Sigma_{f}=0.01 \\
k_{\text {eff }} & =0.99979305228
\end{aligned}
$$

Initial conditions: cosine spatial shape

$$
\phi(0)=1.0 \quad C(0)=8.75181 \times 10^{-4} \begin{aligned}
& \text { measured at } \\
& \text { centerpoint }
\end{aligned}
$$




\section{Critical Configuration 3}

Geometry: Homogeneous, 1-dimension

Reactor width $=20 \mathrm{~cm}$; 8 mesh spaces

For MITKIN and ADC, 1-dimension is simulated by:

Reactor width $=20 \mathrm{~cm}, 8$ mesh spaces $\mathrm{x}$-direction $=1030 \mathrm{~cm}, 2$ mesh spaces y-direction

1 precursor group:

$$
\beta=0.006448 \quad \lambda=0.10
$$

I neutron group:

$$
\begin{array}{rlrl}
v & =4 \times 10^{5} & \Sigma_{a} & =\Sigma_{f}+\Sigma_{c}=0.0835 \\
D & =0.390016 \quad \Sigma_{f} & =0.0467 \\
\nu \Sigma_{f} & =0.0934 & \Sigma_{c} & =0.0368 \\
k_{\text {eff }} & =0.995719743890631
\end{array}
$$

Initial conditions: cosine spatial shape

$$
\phi(0)=1.0 \quad C(0)=5.996654 \times 10^{-3} \text { (centerpoint) }
$$

Critical Configuration 4 (Configuration I of Ref. 19)

Geometry: Homogeneous, 2-dimension

Reactor width: $200 \mathrm{~cm}, 10$ mesh spaces $\mathrm{x}$-direction $200 \mathrm{~cm} ; 10$ mesh spaces $y$-direction

1 precursor group:

$$
B=0.0064 \quad \lambda=0.08 \quad f_{11}=1.0 \quad f_{21}=0.0
$$

2 neutron groups:

$$
\begin{array}{rlrl}
v_{1} & =3 \times 10^{7} & v_{2} & =2.2 \times 10^{5} \\
X_{1} & =1.0 & X_{2} & =0.0 \\
D_{1} & =1.35 & D_{2} & =1.08 \\
\Sigma_{c 1} & =0.00114 & \Sigma_{c 2} & =0.0014069 \\
\nu_{1} & =2.41 & \nu_{2} & =2.41 \\
\Sigma_{f 1} & =0.000242 & \Sigma_{f 2} & =0.00408 \\
\Sigma_{\text {sl }} \rightarrow 2 & =0.0023 & & \\
k_{\text {eff }} \text { not calculated; reactor initially slightly } & \text { supercritical with these constants }
\end{array}
$$


Initial conditions: cosine spatial shape

$$
\phi_{1}(0)=1.0 \quad \phi_{2}(0)=\underset{(\text { centerpoint })}{0.382345 \quad(0)}=3.47419 \times 10^{-3}
$$

Critical Configuration 5 (Configuration 5 of Ref. 19) Geometry: Nonhomogeneous, 2-dimension

\begin{tabular}{|c|c|c|}
\hline 1,2 & $2,2 \cdots$ & 3,2 \\
\hline 1,1 & 2,1 & 3,1 \\
\hline $\mathrm{L}_{1} \longrightarrow$ & \\
\hline
\end{tabular}

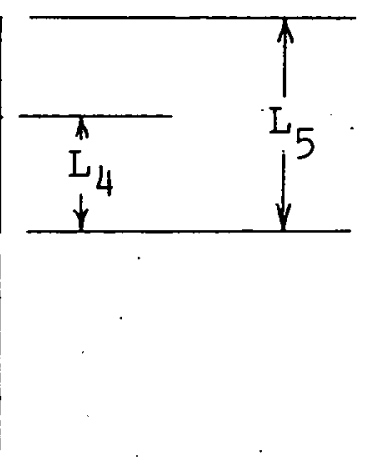

$$
\begin{array}{lll}
\mathrm{L}_{1}=72 \mathrm{~cm} & \mathrm{~L}_{2}=104 \mathrm{~cm} & \mathrm{~L}_{3}=160 \mathrm{~cm} \\
\mathrm{~L}_{4}=32 \mathrm{~cm} & \mathrm{~L}_{5}=80 \mathrm{~cm} & \begin{array}{c}
\text { Regions numbered as } \\
\text { illustrated }
\end{array}
\end{array}
$$

20 mesh spaces $\mathrm{x}$-direction: $\mathrm{h}_{\mathrm{x}}=8.0 \mathrm{~cm}$

10 mesh spaces $\mathrm{y}$-direction: $\mathrm{h}_{\mathrm{y}}=8.0 \mathrm{~cm}$

1 precursor group:

$$
\begin{aligned}
& \beta=0.08 ; \quad \lambda=0.0064 ; \quad f_{11}=0.0 ; \quad f_{21}=1.0 ; \\
& f_{31}=0.0 ; \quad f_{41}=0.0
\end{aligned}
$$

4 neutron groups:

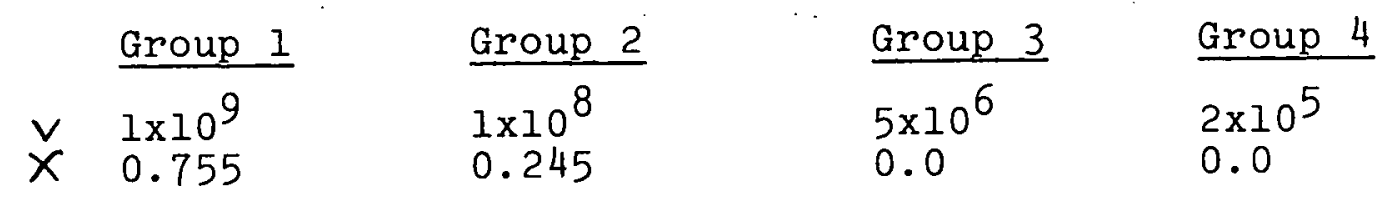

For material in regions $(1,1)$ and $(3,2)$
$\begin{array}{ll}\mathrm{D} & 3.3333 \\ \Sigma_{c} & 0.00065\end{array}$
1.3889
$\Sigma_{c} 1.4507$
0.0005
1.4507
0.83333
0.0045
1.4507
2.0833
0.058
1.4507 


$$
\begin{array}{cccc}
\Sigma_{f} 0.0007 & 0.0009 & 0.0131 & 0.274 \\
\Sigma_{s g \rightarrow 9+1} 0.0586 & 0.0828 & 0.0850 & \text { scattering only to next lower group }
\end{array}
$$

For material in regions $(1,2)$ and $(2,2)$

\begin{tabular}{|c|c|c|c|}
\hline $\begin{array}{ll}\mathrm{D} & 4.1667 \\
\Sigma_{c} & 0.00077\end{array}$ & $\begin{array}{l}2.0833 \\
0.00072\end{array}$ & $\begin{array}{l}1.0753 \\
0.00051\end{array}$ & $\begin{array}{l}0.26247 \\
0.012\end{array}$ \\
\hline$\nu \Sigma_{f} \cdot 0.0$ & 0.0 & 0.0 & 0.0 \\
\hline$\Sigma_{s \rightarrow g+1} 0.0570$ & $\begin{array}{l}0.0822 \\
\text { scatt }\end{array}$ & $\begin{array}{l}0.0847 \\
\text { nly to n }\end{array}$ & \\
\hline
\end{tabular}

$$
\begin{aligned}
& \text { D } 2.7778 \\
& \Sigma_{c} 0.0013 \\
& 1.07 .53 \\
& 0.001 \\
& 0.64103 \\
& 0.0097 \\
& 0.16260 \\
& \nu \quad 1.4507 \\
& 1.4507 \\
& 1.4507 \text {. } \\
& 0.00197 \\
& \Sigma_{s \rightarrow 9+1} 0.0586 \\
& 0.00197 \\
& 0.0262 \\
& 0.115 \\
& 1.4507 \\
& 0.0850 \\
& 0.54
\end{aligned}
$$

For material in regions $(2,1)$ and $(3,1)$

Initial conditions: input by user

Initial condition flux value map for Critical Configuration No. 5. (Map of user input flux values.)

Group $G=1$ (Fast flux group):

$$
\begin{aligned}
& 1 \quad \begin{array}{lllll}
5 & 10 & 15 & 20
\end{array} \\
& 0000000000000000000000 \\
& 100 . . . . \text { e e e .......... . . } 10 \\
& \text { ○. e e d d d d d d e e . . . . . o } \\
& \circ \text {. e d c c b b c c c d d e e.... o } \\
& \circ \text {. e c c b b a b b c d d e e.... o } \\
& \circ \text {. e c c b a a b c c d e e...... o } \\
& 50 . \text { e d c b b b c d e e....... o } 5 \\
& \circ \text {. e e d c c c d e.......... o } \\
& \text { o.. e e e e e e.......... . o } \\
& \text { o.................... . . . } \\
& \begin{array}{cccccccccccccccccccc}
10 & 0 & 0 & 0 & 0 & 0 & 0 & 0 & 0 & 0 & 0 & 0 & 0 & 0 & 0 & 0 & 0 & 0 & 0 & 0
\end{array} \\
& x \text {-direction }
\end{aligned}
$$




$$
\begin{array}{ll}
\text { Group G=1 Legend: } & 0: \quad \phi_{1}=0 \\
& \text { e } 0<\phi_{1} \leqslant .5 \\
& \text { e: } .5<\phi_{1} \leqslant .8 \\
& \text { d: }: 8<\phi_{1} \leqslant 1.0 \\
& \text { c: } 1.0<\phi_{1} \leqslant 1.3 \\
& \text { b: } 1.3<\phi_{1} \leqslant 1.5 \\
& \text { a: } 1.5<\phi_{1}
\end{array}
$$

Group $G=4$ (Thermal flux group) flux map:

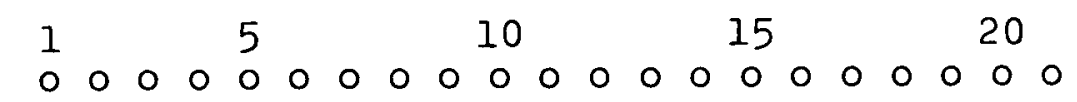

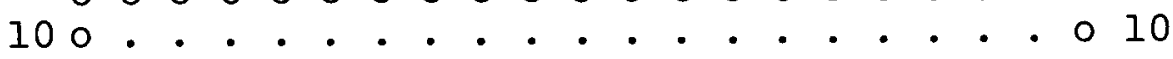

$$
\begin{aligned}
& \text { ○...................... . . . . } \\
& \text { o. } \cdot \text { - } \cdot \text { e e e e } \\
& \text {. } \quad \text { e e e e e e........ o } \\
& 50 . \text {. e e e e e e e e. e e e... o } 5 \\
& \text { - e e e e d e e d a b b c c c d d e.o } \\
& \text { o. e e e e e e d b c c c d d d e e.o } \\
& \text { o... . . . e c d d d e e e...o }
\end{aligned}
$$

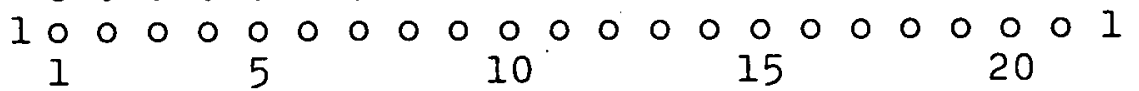

$$
\begin{aligned}
& \mathrm{x} \text {-direction }
\end{aligned}
$$$$
\begin{array}{ll}
\text { Group G=4 Legend: } & 0: \phi_{4}=0 \\
& : 00 \phi_{4} \leqslant .1 \\
\text { e: }: 1<\phi_{4} \leq .2 \\
\text { d: }: 2<\phi_{4} \leqslant .5 \\
\text { c: } .5<\phi_{4} \leq 1.0 \\
\text { b: } 1.0<\phi_{4} \leq 1.5 \\
\text { a: } 1.5<\phi_{4} .
\end{array}
$$

The initial critical condition flux values of Critical Configuration No.5 vary greatly from mesh point to mesh point. In particular, the thermal group flux at neighboring mesh points may vary by as much as a factor of ten. 